\title{
Functional Analysis and Transcriptional Regulation of Oocyte- Specific Nuclear Transporter KPNA7
}

Lei Wang

Follow this and additional works at: https://researchrepository.wvu.edu/etd

\section{Recommended Citation}

Wang, Lei, "Functional Analysis and Transcriptional Regulation of Oocyte-Specific Nuclear Transporter KPNA7" (2015). Graduate Theses, Dissertations, and Problem Reports. 7142.

https://researchrepository.wvu.edu/etd/7142

This Dissertation is protected by copyright and/or related rights. It has been brought to you by the The Research Repository @ WVU with permission from the rights-holder(s). You are free to use this Dissertation in any way that is permitted by the copyright and related rights legislation that applies to your use. For other uses you must obtain permission from the rights-holder(s) directly, unless additional rights are indicated by a Creative Commons license in the record and/ or on the work itself. This Dissertation has been accepted for inclusion in WVU Graduate Theses, Dissertations, and Problem Reports collection by an authorized administrator of The Research Repository @ WVU.

For more information, please contact researchrepository@mail.wvu.edu. 


\title{
Functional Analysis and Transcriptional Regulation of Oocyte-Specific Nuclear Transporter KPNA7
}

\section{Lei Wang}

Dissertation submitted to the

Davis College of Agriculture, Natural Resources and Design at

West Virginia University

in partial fulfillment of the requirements for the degree of

\section{Doctor of Philosophy in \\ Reproductive Physiology}

\author{
Jianbo Yao, Ph.D., Chair \\ Vagner Augusto Benedito, Ph.D. \\ Kenneth P. Blemings, Ph.D. \\ E. Keith Inskeep, Ph.D. \\ Jorge A. Flores, Ph.D.
}

Division of Animal and Nutritional Sciences Morgantown, WV

2014

KEYWORDS: Cattle, rainbow trout, oocyte, early embryogenesis, KPNA7, microRNA, DNA methylation 


\section{Abstract \\ Functional Analysis and Transcriptional Regulation of Oocyte-Specific nuclear transporter KPNA7}

\section{Lei Wang}

The finely orchestrated development and maturation of the oocytes has been the focus of many studies in which oocyte-specific genes essential for folliculogenesis and early embryonic development have been identified. Studies using knockout mice models of Nobox, Sohlh1, Sohlh2, Figla and Lhx8 have revealed that these oocyte specific transcriptional factors form networks in which they regulate the expression of each other and downstream targets. However, little is known about the mechanisms that control the nuclear transport of these essential factors in oocytes and early embryos. The nuclear envelope separates the nuclear and cytoplasmic compartment in the cell and uncouples transcription and translation; therefore, nucleo-cytoplasmic transport is necessary and highly regulated in eukaryotic cells. The soluble proteins that play key roles in mediating the bidirectional trafficking of macromolecules across the nuclear envelope are collectively called Karyopherins (KPNs), with those involved in nuclear import referred to as KPNAs. The first oocyte-specific KPNA gene, KPNA7, was discovered in bovine oocytes; later the homologous genes were found in mice, human and pig. Bovine KPNA7 is highly expressed in germinal vesicle (GV) and metaphase II oocytes, as well as in early stage embryos collected before embryonic genome activation, but is barely detectable in morula and blastocyst stage embryos after the maternal to embryonic transmission. Female mice lacking KPNA7 gene are affiliated with reproductive reduction and sex imbalance by inducing preferential fetal lethality. Induced abnormalities of gene expression (Dppa2, Dppa4 and Piwil2) and epigenetic modifications (down-regulation of histone H3K27me3) are also part of the Kpna7 knock out effects. Although it is known that KPNA7 is a maternal transcript critical for development in mammals, little is known about mechanism of regulation its expression and the nuclear translocation function of KPNA7 protein in bovine embryos or the expression and functions of KPNA7 in rainbow trout.

A series of experiments to study KPNA7 was performed in both cattle and rainbow trout. The first study characterized rainbow trout Kpna7, analyzed its expression, and identified its interacting partners. The cDNA for rainbow trout kpna7 encodes a protein of 519 amino acids, which contains a conserved IBB (importin $\beta$ binding domain) domain and seven ARM (armadillo/beta-catenin-like repeat) motifs. RT-PCR and Western blot analyses revealed that Kpna7 is specifically expressed in mature oocytes. Real time PCR analysis demonstrated that expression of kpna7 mRNA was high in unfertilized oocytes and decreases gradually in early stage embryos until 3 day post fertilization followed by a sharp decreased reaching a level barely detectable in day 4 embryos and thereafter. Using a yeast two-hybrid screening system, two Kpna7-interacting proteins were identified 
from a rainbow trout oocyte cDNA library: Stl3 (rhamnose-binding lectin 3) and an uncharacterized protein. Both genes appeared to be expressed specifically in ovary/testis as revealed by RT-PCR analysis. Co-immunoprecipitation assays confirmed the interaction between Kpna7 and St13, and co-transfection experiments using EGFP-tagged Stl3 showed that Kpna7 facilitated the nuclear transportation of Stl3 through interaction with the predicted NLS cluster at the C-terminus of St13. Results indicated that Kpna7 may function as a unique nuclear transport receptor for oocyte-specific proteins important for early embryonic development.

In the second study, the role of microRNAs and DNA methylation were investigated in regulation of bovine KPNA7 expression during early embryogenesis. Using MicroInspector, an algorithm for detection of possible interactions between microRNAs and target mRNA sequences, 4 binding sites for miR-1296 in the ORF of the bovine KPNA7 mRNA were identified. Expression of miR-1296 was significantly higher in fetal ovary compared to other somatic tissues, and tended to increase in 4-cell and 8-cell stage embryos and decline in morula and blastocyst stages, indicating that miR-1296 is presumably of maternal origin and potentially involved in maternal transcript degradation during the maternal-zygotic transition. To test if miR-1296 regulates bovine KPNA7 protein expression, HEK293 cells were co-transfected with pcDNA3.1-KPNA7 with either pcDNA3.1-miR-1296 or pcDNA3.1 vector. Expression of bovine KPNA7 protein was reduced in cells expressing miR-1296 compared to the control cells, indicating that translation of KPNA7 is repressed by miR-1296. To validate the role of miR-1296 in silencing KPNA7, a luciferase reporter assay was performed using HEK293 cells. miR1296 inhibited KPNA7 expression in a dose dependent manner. Results indicated a functional role of miR-1296 in regulating the expression of maternal oocyte-specific KPNA7 during the maternal to zygotic transition in bovine embryos. To elucidate whether an epigenetic mechanism is involved in the controlled temporal-spatial expression of KPNA7, dynamics of DNA methylation near its transcription starting site (TSS) including proximal promoter, exon 1 and partial intron 1 were examined. Twenty $\mathrm{CpG}$ sites were characterized, which contain one $\mathrm{CpG}$ island of $14 \mathrm{CpG}$ sites in the promoter region ( -210 to -1 upstream of TSS) and 6 CpG sites in exon $1(+1$ to +116$)$. Results showed that $18 \mathrm{CpG}$ sites are hypomethylated $(<20 \%)$ in 16 -cell embryo and blastocyst while 19 sites in somatic tissue are hypermethylated (>80\%). In GV oocyte, four differentially demethylated sites $(-149,-63,-54,+37)$ were discovered compared to somatic samples, indicating that these are potential regulatory sites in the control of tissue-specific expression. The study indicated that although the TSS region of KPNA7 gene is affected by global-demethylation after fertilization, the expression of KPNA7 is not solely controlled by DNA demethylation.

In the third study, the functional role of bovine KPNA7 in meiotic maturation, cumulus expansion and embryonic development was investigated by microinjection of KPNA7 siRNA into cumulus-enclosed GV oocytes. KPNA7 knockdown in oocyte did not affect cumulus expansion but compromised the oocyte competency to reach maturation in vitro and led to retardation of early embryonic development. To further explore the functional network controlled by KPNA7, KPNA7 siRNA injected bovine embryos (8-cell stage) 
were subjected to deep sequencing to identify differentially expressed genes in knockdown embryos compared to the control groups. A total of 1,761 genes were differentially expressed and gene ontology (GO) analysis revealed the enrichment of protein interaction related macromolecule complex component, which is closely related to KPNA7's role as a nuclear transporter.

In summary, the results provide comprehensive evidence that support the idea that KPNA7, as an oocyte-specific maternal nuclear transporter, is essential for oocyte and embryonic development across species. 
Dedication

To 51 


\section{Acknowledgements}

I would like to thank my mentor Dr. Yao for all the help and support.Without him I would never be able to finish my degree. He is and will always be my dearest mentor.

I also want to thank Dr. Inskeep, Dr. Flores and Dr. Daily for years of guidance in the journal club and seminars and in my everyday study they provided me precious support and guidance. Dr. Blemings and Dr. Benedito also provide critical ideas and generous support to my project and my academic study.

I would like to thank Dr. Vernon and Dr. Clemmer for the kind support and granted me more experiences for my career path.

All my lab-mates are my dearest friends and I thank you all for being there.

At last, my gratitude to my grandparents is beyond any words.

Special thanks to Mr. Wuyi Li, who is my family, my best friend, my lifesaver, to whom I would dedicate this work. 


\section{Table of Contents}

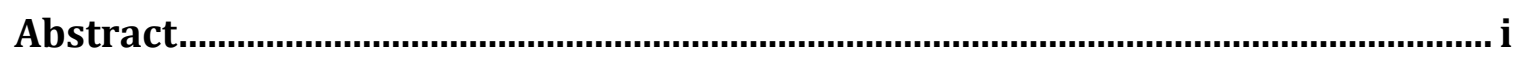

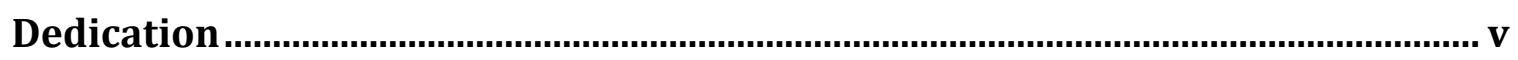

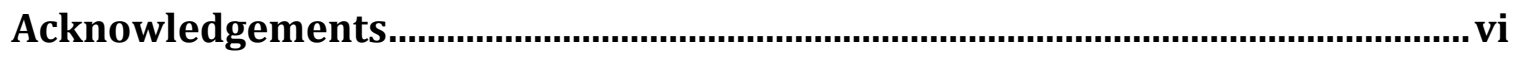

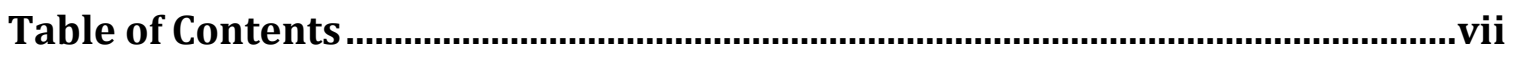

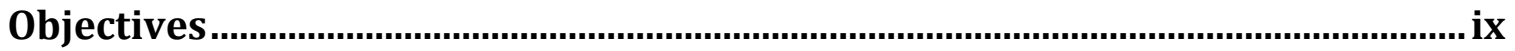

Literature Review........................................................................................................ 1

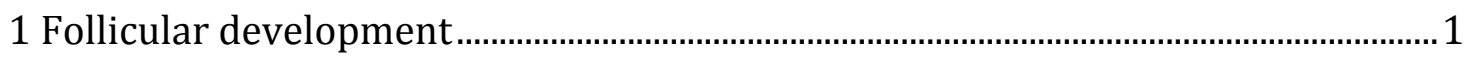

1.1 Important factors in formation and early development of primordial

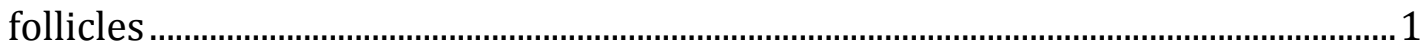

1.2 Important factors critical for proliferation and differentiation of ovarian

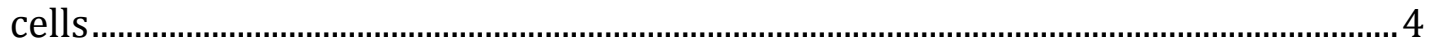

2 Early embryonic development and maternal effect genes............................................ 6

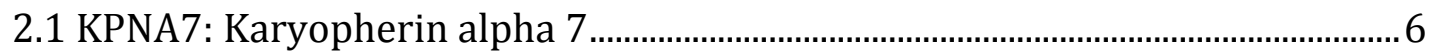

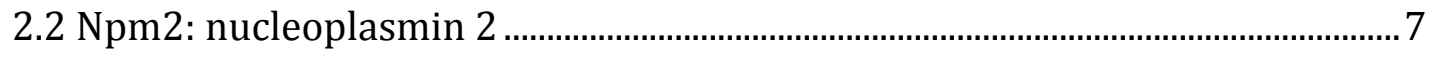

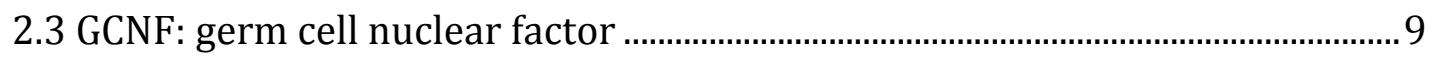

2.4 MATER: maternal antigen that embryos require ..................................................... 9

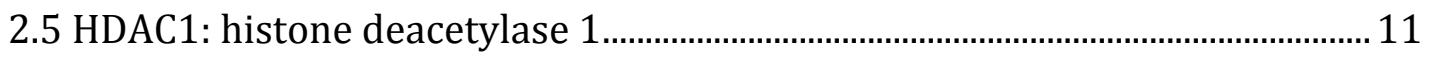

2.6 Nanog: (named after a legendary irish island) ...................................................... 12

3 miRNA regulate the expression of maternal effect genes....................................... 13

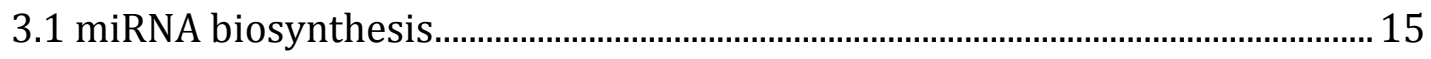

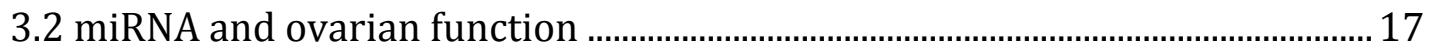

3.3 miRNA in oocyte and early embryonic development .......................................... 20

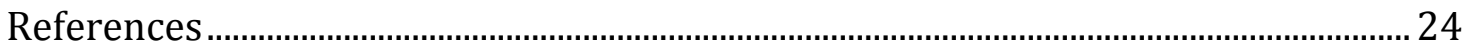

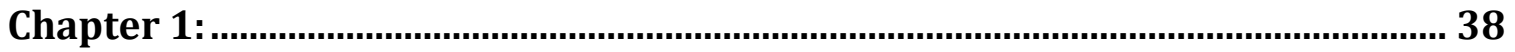

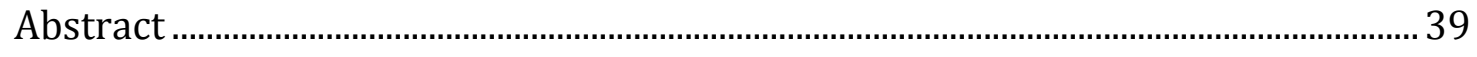

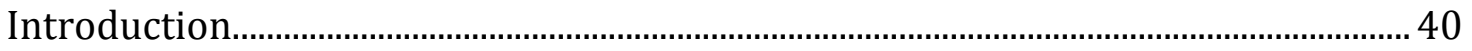

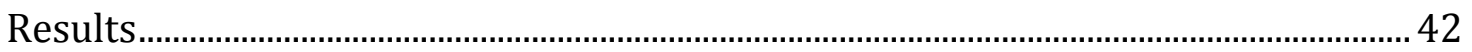




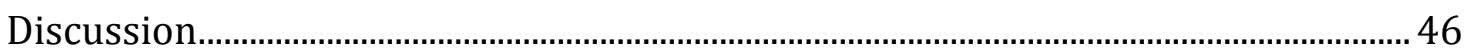

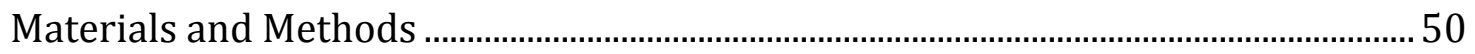

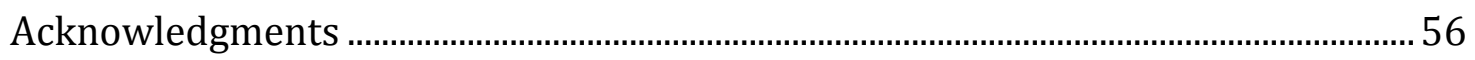

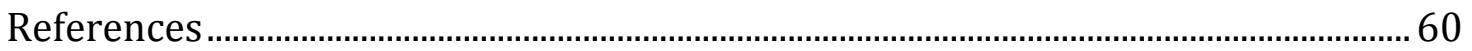

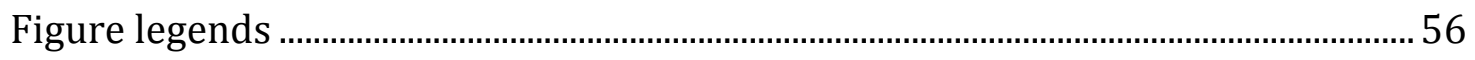

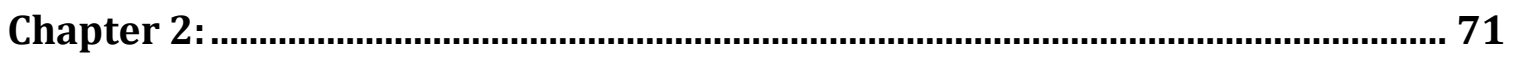

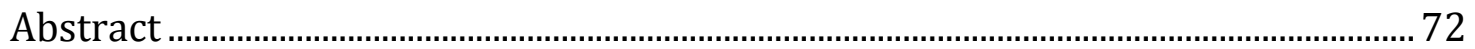

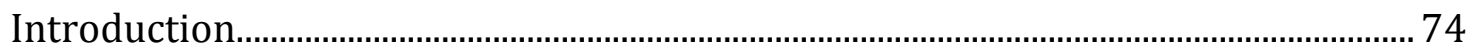

Material and Method............................................................................................................ 76

Results and Discussion .................................................................................................... 79

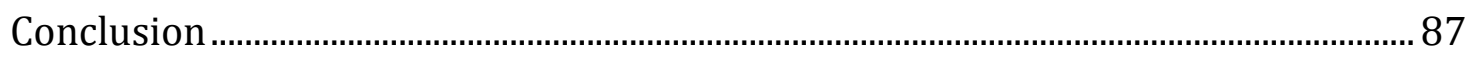

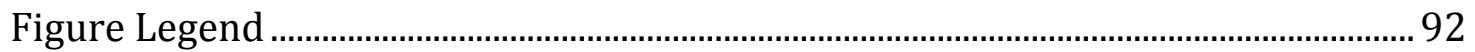

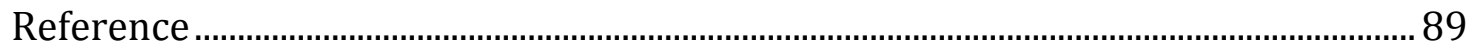

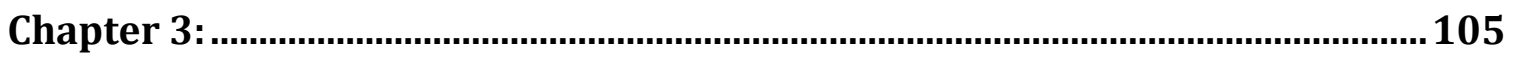

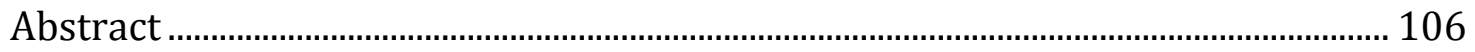

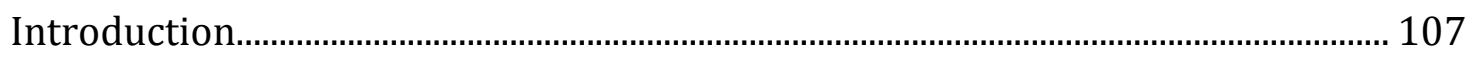

Material and Methods ............................................................................................... 109

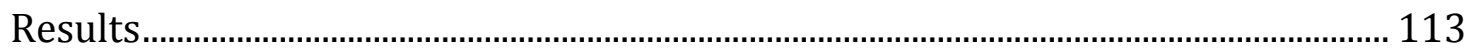

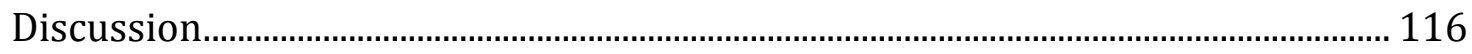

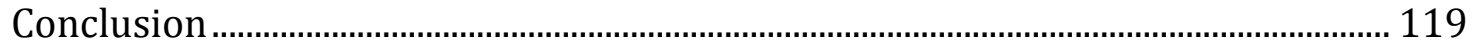

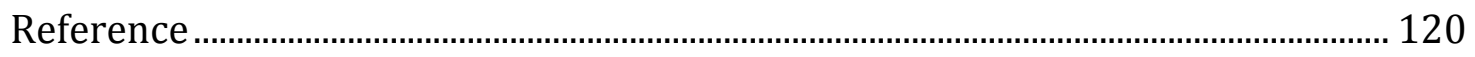




\section{Objectives}

We hypothesized that KPNA7 mediates nuclear transport of key factors required for oocyte maturation, fertilization and early embryonic development both in cattle and rainbow trout and its tissue-and stage-specific expression is under acute control. The general goals of this study are to:

1. Identify the oocyte-specific interaction protein partners of KPNA7.

2. Elucidate mechanisms controlling tissue- and stage-specific expression of KPNA7.

3. Investigate the functional networks that are controlled directly and indirectly by KPNA7.

By utilizing the advantage of both animal models, the specific objectives of studies in each species are described below.

In rainbow trout

1) To identify the KPNA family genes in rainbow trout, and characterize the temporal and spatial expression patterns of KPNA family gene mRNAs during rainbow trout ovarian development and early embryogenesis.

2) To identify the KPNA7 interaction proteins during critical developmental stages of rainbow trout embryos using yeast-two hybridization.

In cattle

1) To test if KPNA7 expression is regulated by methylation alterations on the promoter region of bovine embryos.

2) To determine if microRNA1296 is responsible for degradation of KPNA7 mRNA and inhibition of its translation

3) To determine the phenotypic effect of KPNA7 knockdown on meiotic maturation and initial cleavage divisions

4) To investigate the transcriptome change of KPNA7 knockdown in early embryonic development 


\section{Literature Review}

\section{Follicular development}

Primary follicles are selected from the primordial pool and enter into a growth phase. The oocytes in recruited primordial follicles arrest in prophase of meiosis I, remaining as the follicles grow into primary follicles, secondary follicles, and finally antral follicles. Meanwhile, the oocytes are fully developed and ready for the resumption of meiosis, which is defined as the completion of meiosis I, followed by progression to metaphase of meiosis II, which is the hallmark of oocyte maturation (Nagahama and Yamashita 2008, Rajkovic 2004).

\subsection{Important factors in formation and early development of primordial follicles}

\subsubsection{Figla: Factor in germline alpha}

Figla is a germ cell specific gene identified from mouse oocyte and has been found and studied in humans, interacting with E12 protein that serves as a transcription factor, which controls expression of oocyte-specific zona pellucida genes (ZPs) Figla mRNA is expressed from primordial germ cells all the way to pre-ovulatory follicles (Liang, et al. 1997, Soyal, et al. 2000a). Female Figla knockout mice are infertile as the ZP genes are not expressed and the formation of oocyte is completely abolished. Serial analysis of gene expression revealed that a number of maternal effect genes are directly or indirectly affected by knock out of Figla (Joshi, et al. 2007). Knockout of Figla in female mice led 
to sterility due to impaired meiosis and germ cell apoptosis, meanwhile the Figla null ovary exhibited up-regulation of several testis-specific genes (Hu, et al. 2010b). These data indicate that Figla plays a critical role in activation of oocyte development and repression of testis development to maintain the sex-specific germ cell development. In cattle, Figla shows similar expression profile as in other mammals, which is restricted to gonadal tissues and appears as early as primordial follicle stage (Tripurani, et al. 2013). A study in cattle also revealed a miRNA-mediated controlling mechanism to regulate Figla expression during early embryonic development. As in other maternal effect genes (such as Nobox, NPM2, KPNA7), Figla is expressed in early embryos up to the 8-cell stage in cattle, after maternal to zygotic transition (MZT) from 8 to 16 cell stage when zygotic genome is activated, its expression dropped to undetectable level at morula and blastocyst stage. A miRNA212 binding site (seed region) is present at the 3'UTR of Figla mRNA and this seed region is evolutionary conserved across human, mouse and cattle, indicating the potential role of miRNA212 in post-transcriptional regulation of Figla mRNA. Realtime PCR indicated a similar expression profile of miRNA212 and Figla mRNA, both were decreased after embryonic genome activation suggesting miRNA212 might be a maternal origin factor involved in maternal transcript degradation during the MZT. Luciferase reporter assay in HeLa cells showed specific interactions between miRNA212 and Figla 3'UTR, and studies on in vitro cultured (IVC) bovine embryos revealed that miRNA212 mimic inhibits Figla protein expression (Tripurani, et al. 2013). These studies indicate that Figla is a critical factor for follicular and embryo development and miRNA212 is a potential post-transcriptional regulator of Figla during the MZT in cattle. 


\subsubsection{Nobox: new born ovary homeobox encoding gene}

Nobox is one of the earliest homeobox genes preferentially expressed in germ cells as early as E15.5 (embryos on day 15.5 of gestation) and is present throughout all stages of folliculogenesis in mice (Rajkovic, et al. 2004, Suzumori, et al. 2002). Nobox deficiency in female ovary led to normal development of primordial follicles, however, further development was arrested at the transition from primordial to primary follicle. Therefore Nobox knockout female mice suffered severe infertility phenotype as oocytes were depleted from the ovary (Rajkovic, et al. 2004). Nobox depletion decreases of a number of very important oocyte-specific genes (such as Mos, Oct4, Zar1, Gdf9, Bmp15, H1oo, Rfp14, Fgf8 and Dnmtlo), some of which have been shown to be essential in oogenesis and early embryogenesis, therefore Nobox is a master regulator in follicular development (Rajkovic, et al. 2004). In cattle, Nobox was characterized and revealed to be important even after fertilization during early embryonic development (Tripurani, et al. 2010a). Knockdown of Nobox in early bovine embryos using siRNA caused downregulated of genes led to early development and the blastocyte rate was significantly reduced. Nobox expression has been studied in cattle oocyte and early embryos. It has high expression in germinal vesicle and metaphase II stage oocytes and all the way to 4cell stage embryos, after embryonic genome activation (8-16 cell stage in cattle). However, Nobox expression drops at the 8-cell stage and is barely detectable at the morula and blastocyst stages (Tripurani, et al. 2010a). The sudden drop of Nobox mRNA at the stage of embryonic genome activation was later found to be controlled by miRNA196a (Tripurani, et al. 2010a). miRNA196a recognizes and binds to the 3'UTR of bovine Nobox miRNA and its expression tends to increase at 4-8 cell stages. Co- 
expression of Nobox and miRNA196a in HeLa cells inhibited the expression of Nobox protein compared to control cells without miRNA196a. Specific binding of miRNA296a to Nobox mRNA was supported by luciferase assay with mutation on the predicted binding site. Furthermore, ectopic expression of miRNA196a mimics in bovine early embryos reduced Nobox protein expression. These data indicate that miRNA196a mediates the degradation of the untranslated maternal Nobox mRNA, which is very critical for early embryonic development.

\subsection{Important factors critical for proliferation and differentiation of ovarian cells}

\subsubsection{TGF- $\beta$ : tumor growth factor beta}

Folliculogenesis involves not only the careful orchestration of developmental programs in oocytes and surrounding somatic cells, but also the intimate interaction between the two cell types (Matzuk, et al. 2002). Members of the transforming growth factor beta (TGF- $\beta$ ) super family function as paracrine and autocrine factors to exert important effects regulating developmental processes in the sub-cellular environment (Bilezikjian, et al. 2006). Members of the TGF- $\beta$ superfamily have been characterized into three major subfamilies, based on their structural homologies: 1. activins/inhibins; 2. bone morphogenetic proteins (BMP); and 3. TGF- $\beta$ (Heldin, et al. 1997, Massague 1998). As commonly expressed in mammalian ovary, TGF- $\beta 1$ and the other isoforms, $\beta 2$ and $\beta 3$ have been detected in several fish species including: rainbow trout, goldfish, carp, hybrid striped bass, European eel and Siberian sturgeon (Hardie, et al. 1998, Harms, et al. 2000, Laing, et al. 2000, Mulheron, et al. 1991, SchmidP and vanderPuttenH 1994, Zhan and Jimmy 2000). 


\subsubsection{BMP15 (bone morphogenetic protein 15) and GDF9 (growth differentiation factor 9)}

BMP15 and GDF9, members of bone morphogenetic proteins, have captured increasing attention and changed traditional views on active roles of the oocyte in controlling folliculogenesis (Coskun and Lin 1994, Feng, et al. 1988, Singh, et al. 1993). As transcription factors, GDF9 and BMP15 are thought to play active paracrine roles in controlling the differentiation of surrounding somatic cells by oocytes (Elvin, et al. 1999a, Matzuk, et al. 2002, Vitt and Hsueh 2002). GDF9 has been cloned and detected in diverse mammalian species (Aaltonen, et al. 1999, Bodensteiner, et al. 1999, Dong, et al. 1996a). It is expressed exclusively in oocytes from early primary follicles to early antral follicles; after fertilization it can still be detected up to the 8-cell stage in cattle (Sendai, et al. 2001). In GDF9 knockout mice, the disruption of GDF9 expression led to infertility due to arrested development at the primary follicle stage with the absence of the thecal cell layer and incompetence in oocyte meiotic division (Carabatsos, et al. 1998, Dong, et al. 1996a). Also in antral follicles, FSH induces granulosa cell differentiation, which is partly blocked by GDF9. Therefore, the cumulus cell layer was undifferentiated and the expansion of cumulus cells surrounding the oocyte was disrupted in GDF9 null mice (Elvin, et al. 1999b). BMP15 (GDF9b) is a homolog of GDF9, with which it shares 52\% amino acid identity. BMP15 is an oocyte-specific gene with a similar expression pattern as GDF9 (Dube, et al. 1998, Laitinen, et al. 1998). However, BMP15 null mice show that BMP15 is not as essential for folliculogenesis as its counterpart GDF9. Disruption of 
BMP15 expression did not greatly affect early folliculogenesis, but to some degree it can reduce the ovulation rate (Yan, et al. 2001).

\section{Early embryonic development and maternal effect genes}

\subsection{KPNA7: Karyopherin alpha 7}

Transportation of nuclear proteins (such as Nobox) into the nucleus through nuclear pores is a vital event in development of gametes and early embryos (Geles and Adam 2001, Mason, et al. 2002, Tejomurtula, et al. 2009). The KPNA protein family, which contains 7 family members, is one of the major players in translocation of macromolecules through an active, energy-dependent nuclear import system (Chook and Blobel 2001, Otis, et al. 2006) and only KPNA7 is specifically expressed in bovine, porcine and murine oocyte (Hu, et al. 2010a, Tejomurtula, et al. 2009, Wang, et al. 2011). Knockout of KPNA7 in female mice caused fecundity reduction and sex imbalance by inducing preferential fetal lethality, abnormal expression of important maternal effect genes and epigenetic modifications (Hu, et al. 2010a). In bovine and porcine studies, knockdown of KPNA7 in early embryos lead to arrested embryo development and an expression pattern of a maternal effect factor, which is depleted after MZT (Tejomurtula, et al. 2009, Wang, et al. 2011). It was hypothesized that KPNA7 is regulated by microRNA targeting for silencing and/or degradation during MZT, because four binding sites for miRNA1296 on the ORF of bovine KPNA7 mRNA were identified. Expression of miRNA1296 was significantly higher in fetal ovary compared to other somatic tissues, and tended to 
increase in 4-cell and 8-cell stage embryos and decline in morula and blastocyst stage embryos, similar to other miRNAs found to be involved in maternal factor degradation such as miRNA196a, 212 and 181a. Therefore miR-1296 is presumably of maternal origin and potentially involved in maternal transcript degradation during the maternalzygotic transition. To test if miRNA1296 regulates bovine KPNA7 protein expression, HeLa cells were co-transfected with KPNA7 with either miRNA1296 or control vector. Expression of bovine KPNA7 protein was reduced in cells expressing miRNA1296 compared to the control cells, indicating that translation of KPNA7 is repressed by miRNA1296. Luciferase reporter assay with mutation of 4 binding sites on KPNA7 mRNA revealed one of the binding sites (1156-1176bp) is the primary interaction site between miRNA1296 and KPNA7 mRNA. This results indicates a potential functional role of miRNA1296 in regulating the expression of maternal oocyte-specific KPNA7 during the maternal to zygotic transition in bovine embryos (Wang, et al. 2013b). Further study is needed to investigate the in vivo role of miRNA1296 in bovine embryonic development.

\subsection{Npm2: nucleoplasmin 2}

NPM2 is a nuclear chaperone involved in decondensation of sperm chromatin, first identified in Xenopus oocytes (Philpott, et al. 1991). Mammalian NPM2 orthologs have been discovered in human, mouse, cattle and among others. In mouse oocytes, NPM2 segregates in nuclei and stays there through the pre-implantation embryos. In mouse NPM2 knockout led to impaired development of the 2-cell stage as a result of reduced cleavage rate. NPM2 null embryos showed high chromatin abnormalities including loss 
of heterochromatin and increased acethylated $\mathrm{H} 3$ around the nuclei, all of which led to embryo death after 50 hours post-fertilization (Burns, et al. 2003). On the other hand, overexpression of NPM in bovine oocytes after somatic cell nuclear transfer results in normal embryos, which lead to higher pregnancy rate after transfering to normal females (Betthauser, et al. 2006). These results indicate that NPM is critical for chromatin reprogramming and normal development of early embryos. In cattle, NPM2 mRNA and protein was most abundant in GV and MII oocytes and decreased from zygote to blastocyst without re-expression even after embryonic genome activation. In persistent bovine follicles, NPM2 mRNA was very low compared to normal. These particular expression profiles indicate that NPM2 is a maternal effect factor associated with oocyte quality and it might potentially serve as a marker for oocyte developmental competence. (Lingenfelter, et al. 2011). Most importantly, miRNA181a was found to interact with NPM2, which might lead to the silencing and/or degradation of NPM2 mRNA during early embryogenesis. miRNA181a was expressed in all oocyte samples and increased in 4-16 cell embryo stages, coincident with the time of embryonic genome activation in cattle when NPM2 decreased. Apart from regulating a maternal effect gene during early embryonic development, miRNA181a, was involved in granulosa cell proliferation and embryonic stem cell differentiation (Gu, et al. 2008). Further investigation showed binding site of miRNA181a on 3'UTR of NPM2 and evidence of translational silencing of NPM2 by miRNA181a in bovine embryos (Lingenfelter, et al. 2011). This was the first report of a miRNA involved in regulation of a maternal effect oocyte-specific gene in mammals. 


\subsection{GCNF: germ cell nuclear factor}

GCNF was first identified in mouse and its expression was restricted to developing germ cells (Chen, et al. 1994). GCNF works as a primary regulator of the paracrine interaction between oocyte and somatic cells. Oocyte-specific GCNF knockout mice displayed longer estrous cycles, aberrant steroidogenesis and an abnormal gene expression patterns of BMP15 and GDF9, all of which result in lower fertility. It is known that GCNF interacts with promoter sequences of BMP15 and GDF9 and repress their reporter activities (Lan, et al. 2003). GCNF is a primary repressor of Nanog, which is essential for early embryogenesis and maintenance of pluripotency (Gu, et al. 2005). An in silico study revealed that miRNA181a is expressed from the intron of GCNF (germ cell nuclear factor) gene on the non-coding strand and is predicted to target the GCNF gene through 3'-UTR binding site. Interaction between miRNA181a and GCNF is supported by the correlated expression of miRNA181a and GCNF in different embryonic stem cell-line (Gu, et al. 2008). GCNF directly targets the promoter region of miRNA302 and inhibits miRNA302 expression in embryonic stem cells; indirectly, inhibition of cyclin D1, which is targeted and repressed by miRNA302, is removed by GCNF (Wang, et al. 2014). These findings support a paradigm of a negative feedback loop that GCNF participates in embryonic stem cell differentiation as a major repressor through the interaction with particular miRNAs and indirectly regulating expression of downstream targets $(\mathrm{Gu}$, et al. 2008).

\subsection{MATER: maternal antigen that embryos require}

The first important developmental transition that occurs following fertilization is 
activation of the embryonic genome, during which time transcripts expressed from the zygotic/embryonic genome replace maternal transcripts that direct initial development [49]. This crucial transition occurs during the first few post- fertilization cell cycles in a species dependent manner. Embryonic genome activation occurs at roughly the 2-cell stage in mice, 4- to 8-cell stage in humans, and 8- to 16-cell stage in cattle [50,51]. The transition from maternal to embryonic control of development is characterized by degradation of maternal transcripts and proteins, sensitivity to transcriptional inhibitors (e.g. $\alpha$ - amanitin), and a dramatic increase in transcriptional activity from the embryonic genome [52]. Several oocyte-expressed genes are known to be essential for initial stages of embryonic development. One such gene is known as MATER (maternal antigen that embryos require). MATER was identified initially as an oocyte antigen in a mouse model for premature ovarian failure [53]. Deficiency of MATER does not affect the number of primordial follicles or the overall ovarian architecture. MATER-deficient females ovulate the same number of eggs as wild-type controls, and resulting zygotes progress through the first cleavage division. However, MATER-deficient embryos arrest in development at the 2-cell stage in mice, indicating that it is a key maternal gene whose expression is required for embryonic development [54]. Two additional maternal effect genes, Zygote Arrest 1 (ZAR1) and NPM2 are required for normal early embryonic development [3, 55]. ZAR1 mutant females are infertile and show a defect in embryogenesis at the one cell stage with a pronounced inhibition of syngamy [55]. Fewer than $20 \%$ of embryos from ZAR1 mutant females advanced to the 2-cell stage and displayed markedly less transcription [55]. Xenopus NPM2 has been shown to decondense sperm DNA in vitro [20]. NPM2-null mutant mice are subfertile with pronounced defects in preimplantation 
embryonic development [3].

\subsection{HDAC1: histone deacetylase 1}

HDAC1 is a member of the histone deacetylase family and plays a key role in eukaryotic gene expression. To date, 18 classic HDACs have been discovered and classified into two major groups based on their sequence identity and protein-protein interaction partners (Thiagalingam, et al. 2003). As one of the components of nuclear corepressor complexes, HDAC1 is found to be tightly related to transcription machinery (Hassig, et al. 1998). From studies investigating human oocyte transcriptome, HDAC1 was found to be significantly overexpressed in oocyte, which indicates the special need and functions HDAC1 plays in oocyte genetics (Assou, et al. 2006, Kocabas, et al. 2006). In somatic cells, histone acetylation transforms condensed chromatin into a relaxed stage which is associated with a transcription active state and deacetylation suppresses the transcription activity (Rice and Allis 2001). However, in GV oocyte, histone deacetylation is not related to transcriptional repression, whereas it is essential for the changes in chromatin configuration and chromosome segregation (Akiyama, et al. 2006, De La Fuente, et al. 2004, Miyara, et al. 2003). Inhibition of histone deacetylase activity results in aneuploidy during meiosis and high embryonic death during pregnancy (Akiyama, et al. 2006). After fertilization, the dominant deacetylase in preimplantation embryos is $\mathrm{HDAC} 1$ with a major target for deacetylation being $\mathrm{H} 4 \mathrm{~K} 5$, which is a biomarker indicative of rapid transcription activity when it is acetylated. Posttranslational modifications by acetylation of the N-terminal portion of histones composing the nucleosome are involved in genome reprogramming. These modifications alter the higher-order chromatin structure to render 
the DNA accessible to the regulatory and transcriptional machinery.

Although knowledge of maternal control of early embryonic development in mice has been enhanced through discovery and knockout of the above genes, our overall understanding of oocyte regulation of early embryonic development and the genes involved is less than complete, particularly in livestock species.

\subsection{Nanog: (named after a legendary irish island)}

Nanog is a transcription factor harboring a homeobox domain first identified in mouse embryonic stem cells and is known as an important pluripotent factor (Chambers, et al. 2003). It is restricted to the inner cell mass of early embryos and embryonic stem cells of human, mouse, rat, pig, sheep and cattle. Expression of Nanog is not consecutive. It starts from inner cell mass of blastocyst followed by a long silence during embryogenesis until the formation of primordial germ cells (PGCs). Nanog expression increases during the development of PGCs until they enter mitotic arrest and stop proliferating. This unique expression pattern indicates that Nanog is specific for nuclear reprogramming during germ cell development (Yamaguchi, et al. 2005). Nanog knockout ES cells spontaneously differentiate into extraembryonic endoderm lineage and overexpression of Nanog is sufficient for maintaining the self-renewal capacity of ES cells (Chambers, et al. 2003). Oct4 and Sox 2 have been shown to regulate Nanog expression by binding to promoter region of Nanog (Rodda 2005). More biological pathways have been revealed as part of the complicated regulatory network controlling stage-specific expression of Nanog including miRNA mediated post-transcriptional attenuation. By comparing with the bovine miRNA transcriptome of early blastocyst with hatched blastocyst, miRNA218 
was found to be down-regulated after hatching moreever its expression is restricted to the inner cell mass, which is coincident with the expression pattern of Nanog. Luciferase assays revealed that the interaction between miRNA218 and Nanog is genuine and lead to post-transcriptional attenuation of Nanog protein. When interfering with FGF/MAP kinase pathway by adding exogenous FGF4 in the embryo culture medium, the development of the blastocyst was not affected, however, decreased expression of Nanog and increased miRNA218 was observed (Goossens, et al. 2013). Exogenous supplementation of FGF4 is well known to promote the differentiation of bovine blastocyst towards endoderm linage through a proposed Nanog repression mechanism (Dorey and Amaya 2010). The discovery of the positive correlation between miRNA218 expression and FGF4 stimulation evidenced the repression mechanism of Nanog directly targeted by miRNA218 in response of the FGF signaling at the beginning of the endoderm differentiation. Retinoic acid treatment of embryonic stem cells leads to differentiation towards ectodermal lineage with elevation of miRNA134. Nanog was proved to be targeted directly by miRNA134 and this interaction lead to down-regulation

of Nanog protein. Nanog is not present in cells from ectodermal lineage, so the interaction between miRNA134 and Nanog revealed another regulatory pathway of controlling Nanog expression. Further study is needed to investigate the functional network between different miRNA pathways in repression or activation of Nanog.

\section{3 miRNA regulate the expression of maternal effect genes}

Many agriculture species of commercial importance suffer from problems related to egg quality and early embryonic development. In dairy cattle industry, it is well known that in 
vivo-derived embryos has higher developmental competency. Only $5 \%$ of in vivo-derived embryos will go through abortion whereas, higher than $50 \%$ of abortion rate to first service is observe in artificially inseminated cows. In bovine IVF culture system, blastocyst rate is around $30 \%-50 \%$ and after embryo transfer, abortion rate is $8 \%$ to $13 \%$, with the greatest embryonic loss occuring around day 8 (Diskin, et al. 2011, Evans and Walsh 2011, Moore and Thatcher 2006). The newly fertilized embryos undergo dramatic morphologic and genetic changes mainly during MZT (Van Soom, et al. 1992). As the total amount of cytoplasmic materials is constant within the embryos, the rapid cell division and development before MZT is driven by the maternal-derived factors, accumulated inside the oocyte. During early embryonic development, when transcriptional activity is repressed, these maternal-oriented transcripts will be translated into functional proteins or stored for later recruitment leading to embryonic genome activation or being degraded after MZT (Memili and First 2000). Therefore, oocyte quality is highly related to embryo competence and there is a definitive and imperative need to understand the functions of oocyte-derived factors and how their translation and degradation are controlled at different stages of oogenesis and embryogenesis. A growing body of evidence indicates that microRNAs (miRNA) are a major mediators involved in translational control during oocyte development and early embryogenesis (Hossain, et al. 2012). miRNAs are a group of small non-coding RNAs with 18-24 nucleotides and were first discovered in C. elegans (Lee, et al. 1993). Since then, miRNAs have been found to have multiple biological regulatory effects, mediated by recognizing different sites of target genes either on mRNA or on the promoter sequence in the genome (Bartel 2009, Place, et al. 2008). Recent studies have implicated the regulatory functions of miRNAs 
from ovarian follicular development to early embryonic development. Specific gene targets of miRNA have been found to be involved in critical biological pathways, such as chromatin remodeling, self-renewal proliferation, transcription initiation and nuclear transportation. After fertilization, the embryo spontaneously orchestrates the abovementioned developmental processes. A better understanding of the physiological roles played by maternal factors could help us to combat the low efficacy and high embryonic loss in the dairy industry. Most importantly, the functions of miRNA in controlling translation and degradation of the maternal genes will be discussed with emphasis on miRNA biogenesis, specific target genes and exosomal transportation of miRNA in ovarian and embryonic tissues.

\section{1 miRNA biosynthesis}

By complementary binding to target sequences on specific mRNA or genome regions, miRNAs achieve their functions to regulate gene expression and translation through translational repression, mRNA degradation or posttranscriptional activation (Bartel 2009, Vasudevan 2012). They are encoded by the genome and are produced from intergenic regions, introns or exons of protein coding genes (Bartel 2004). Most miRNAs have their own enhancers and promoters, which indicate that their expression could be controlled by transcriptional factors, DNA methylation or other mechanisms found in protein coding genes (Corcoran, et al. 2009, Han, et al. 2007, Rodriguez, et al. 2004). Pathways for processing miRNA are conserved through different species and are mostly transcribed by RNA polymerase II or in some cases RNA polymerase III (Borchert, et al. 2006, Winter, et al. 2009). At the beginning of transcription, primary 
miRNA (pri-miRNA) is transcribed by a corresponding RNA polymerase (II or III) and folded into the characteristic hair-pin structure through the complementary regions on the pri-miRNA sequence (Figure 1). While still in the nucleus, pri-miRNA is then processed by protein complex RNase III Dorsha and DGCR8 (diGeorge syndrome critical region 8) to form precursor miRNAs (pre-miRNAs) of about $70 \mathrm{nt}$. With the help of Exportin5, a RanGTP-dependent nuclear transporter, pre-miRNA is translocated from the nucleus to the cytoplasm (Bohnsack, et al. 2004). Once in the cytoplasm, protein complex RNase III Dicer and TEBP recognize pre-miRNA and then cleave the loop and the guide strand. Meanwhile, the protein complex also cleave the passenger strand of the pre-miRNA into its mature length (Chendrimada, et al. 2005). Recruited by TEBP, Ago2 now binds to the passenger strand and both are loaded into an RNA-induced silencing complex (RISC), where the passenger strand matures and can achieve its functions through complimentary binding to the target sequences (Khvorova, et al. 2003). About $40 \%$ of miRNAs are located within intronic regions of protein-coded genes. The production of some of the miRNAs originates from short intronic region follows a different pathway, the mirtron pathway, which is Dorsha/DGCR8 independent (O'Carroll and Schaefer 2013, Westholm and Lai 2011). In mirtron pathway, short introns with hairpin potential are spliced by spliceosome and with the help of debranchase enzyme, a pre-miRNA mimic is generated independently of Drosha/DGCR8 processing. Pre-miRNA (mirtron) is directly exported and processed by Dicer and RISC following the classical pathway. The mirtron pathway is found to be conserved across species including cattle, however, because the mirtron pathway is newly discovered, only a few mirtron miRNAs have been identified, which 
compose a small population of miRNAs (Berezikov, et al. 2007, Davis and Hata 2009, Glazov, et al. 2009).

\section{2 miRNA and ovarian function}

\subsubsection{Presence of miRNA in bovine ovary and early embryos}

The ovary is composed of several cell types, including the oocytes and a number of somatic cells such as granulosal, thecal, luteal and cumulus cells. Following the signals from the hypothalamic-pituitary-gonadal axis, these cells coordinately respond to endocrine and paracrine factors, which eventually work back on the cells and regulate the expression and dynamics of the gene network in order to achieve the ovarian cycle. Each step of the ovarian cycle is distinguished by its unique gene expression profile changing sequentially between follicle recruitment, selection, atresia, ovulation, luteinization and luteolysis. As a major regulator of gene expression, miRNAs play important roles in follicle development. A significant amount of studies, use different evaluation methods of microarray, Taqman assay, real-time PCR and next-generation sequencing, to test the miRNA expression profiles during folliculogenesis in a variety of samples from different species. Here, we have summarized the studies about bovine miRNA expression in ovarian and early embryonic development. It is revealed that let7a, $7 \mathrm{~b}$ and $7 \mathrm{c}$ are the most commonly expressed and the most abundant miRNA in bovine fetal and adult ovary. The second most abundant group of miRNAs are miRNA143, miRNA106, miRNA26a, miRNA21 and miRNA126. These large-scale studies not only identified the

population of bovine miRNA, but also investigated the miRNAs originating from the 
oocyte, different ovarian cells and different embryo stages. However, less is known about specific roles of miRNAs in bovine studies.

\subsubsection{Involvement of miRNA in follicular growth}

\section{Intracellular miRNAs in oocyte and somatic cells}

The most important functions of ovary are: first to produce viable oocytes resulting in successful fertilization and healthy embryos and second, to form a functional corpus luteum (CL) which is critical in the maintenance of pregnancy (Richards 2007). These biological processes are highly dependent on the functions of many somatic cells including granulosal cells. During oocyte development, granulosal cells play important roles in preparing the follicle in acquiring competence to respond to an LH surge, which initiates resumption of meiosis, cumulus cell expansion, luteinization and progesterone production. During the follicular phase, development and proliferation of granulosal cells is controlled by FSH, triggering development of immature pre-antral follicles into preovulatory follicles. In rat, exposing granulosal cell to FSH for 12 hours led to altered expression of 31 miRNAs. Among them, expression of miRNA29a and miRNA30d underwent time-dependent manner with increase in expression of the FSH supplemented culture, which indicate a prolonged effect of miRNA during follicular genesis (Yao, et al. 2010b). Initiated by LH surge, differential gene expression profiles during these orchestrated series of events within granulosa cells, are observed and more and more evidence indicate the involvement of miRNA in the behavior of granulosa cells (Russell and Robker 2007, Yao, et al. 2010a). Both precursor and mature miRNA132 and miRNA212 were significantly up-regulated after the LH surge. In silico analysis 
identified 77 potential mRNA targets of miRNA 132 and 212 in granulosal cells, it was suggested that the post-transcriptional regulatory effect of miRNA-mediated gene expression was important in granulosa cells. Knockdown of miRNA132 and miRNA212 showed decreases in protein, but no change in mRNA of C-terminal binding protein 1 (CTBP1), which is known to interact with steroidogenic factor 1, a transcription factor known to modulate promoter activity of steroidogenic related genes (Dammer and Sewer 2008, Fiedler, et al. 2008). After ovulation, the CL, which is derived from the ovulated follicle requires intense angiogenesis and produces progesterone essential for the maintenance of early pregnancy. Formation of the CL is also regulated by miRNAs. In mouse, absence of miR17-5p and let7b, resulted in impaired CL formation through targeting the expression of the antiangiogenic factor tissue inhibitor of metalloproteinase 1 (Otsuka, et al. 2008).

\section{Extracellular miRNAs in follicular development}

Presence of distinct set of intercellular miRNAs in oocytes and different types of somatic cells has implicated their key regulatory roles in all stages of bovine follicular development. Moreover, studies have indicated that extracellular miRNAs as playing critical roles in regulation of bidirectional communication between the oocyte and somatic cells (da Silveira, et al. 2012, Sohel, et al. 2013). Numerous studies in different species have demonstrated that microvesicles and exosomes released from many cell types serve as endocrine and/or paracrine regulatory factors to influence recipient cell phenotypes (Properzi, et al. 2013, Valadi, et al. 2007). In the horse, 41 and 79 miRNAs were detected in follicular fluid from exosomes and microvesicles origin, respectively. 
Uptake of fluorescent-labeled microvesicles by granulosal cells was observed after reintroducing labeled follicular fluid to the same antral follicle. Furthermore, bioinformatics analysis demonstrated that miRNAs found in microvesicles and exosomes isolated from follicular fluid was present within granulosal and cumulus cells, and the populations of miRNAs differed among different aged females (da Silveira, et al. 2012). In humans, several miRNAs were found to be transferred via microvesicles present in follicular fluid, some of them (miRNA132, 320, 520c-3p, 24 and 222) regulate estradiol production and some (miRNA24, 193b and 483-5p) regulated progesterone production in a human granulosa-like tumor cell line (Sang, et al. 2013). In cattle, exosomal and nonexosomal fractions of extracellular microRNAs in follicular fluid were related to bovine oocyte developmental competence (Sohel, et al. 2013). Follicular fluid from follicles containing matured or immature oocytes was isolated separately. Exosomal and nonexosomal fractions were separated from the corresponding follicular fluid pools and miRNA were found in both fractions. Differentially expressed extracellular miRNAs were identified with 25 miRNAs in exosomes and 30 miRNA in non-exosomal fraction. Uptake of exosomes into follicular cells was observed in primary culture of bovine granulosa cells (Sohel, et al. 2013). These studies demonstrated an important role of miRNA population in the follicular environment in follicular development and oocyte competence.

\section{3 miRNA in oocyte and early embryonic development}

The finely orchestrated development and maturation of the oocyte have been the focus of many studies in which essential oocyte-specific genes have been identified (Sun, et al. 
2008, Zheng and Dean 2007b). A number of oocyte-specific factors were identified and proved to play an essential role in mammalian folliculogenesis and early embryonic development (Pangas and Rajkovic 2006). These genes encode for many factors involved in chromatin remodeling, self-renewal proliferation, transcription initiation and nuclear transportation. Aberrant expression of these essential genes led to abnormalities in multiple developmental stages from failure of oocyte meiosis resumption to disruption of early embryonic development (Dong, et al. 1996b, Rajkovic 2004, Soyal, et al. 2000b, Virant-Klun, et al. 2013). Therefore, acute control of the expression of these factors is critical for normal follicular and embryo development. As such, miRNAs with temporaland spatial-specific expression patterns are considered as major mediators in regulation of the oocyte-specific genes.

Recent studies implicated miRNAs in maternal transcript degradation during MZT of different species. Such as miRNA430 in zebrafish, miRNA427 in Xenopus and miRNA21 in rainbow trout are present prior to embryonic genome activation. Further studies revealed more evidence supporting the idea that such miRNAs are responsible for degradation of hundreds of maternal transcripts (Bazzini, et al. 2012, Giraldez, et al. 2006, Lund, et al. 2009, Ramachandra, et al. 2008). In conditional DGCR8 knockout mice, which blocks the miRNA biogenesis pathway specifically, although the litter size was reduced, oocyte maturation and blastocyst rate remained the same (Suh and et al. 2011). Moreover, 3'UTRs of transcripts, which were upregulated in Dicer1 knockout oocytes were not enriched (Ma, et al. 2010). Results of these two studies in knockout mouse model weakened the importance of miRNA during early embryo development. However, 
these studies focused only on 3'UTR targeting miRNAs generated by the canonical Dicer- and DGCR8- dependent pathway; other miRNAs targeting ORF of mRNA and/or generated by non-canonical DGCR8-independent pathway were not investigated. The important impact of miRNAs on early development is supported by studies in zebra fish, frog and rainbow trout, but less so by the studies in mouse regarding to miRNAs from certain biogenesis pathway. Given that very few studies have investigated the role of miRNAs on specific targets during the bovine maternal-to-zygotic transition, in this section, current knowledge is summarized based on combined data from mouse and other species about how selected oocyte-specific factors work with specific miRNAs and their functional roles potentially relevant to develop competence in cattle. 


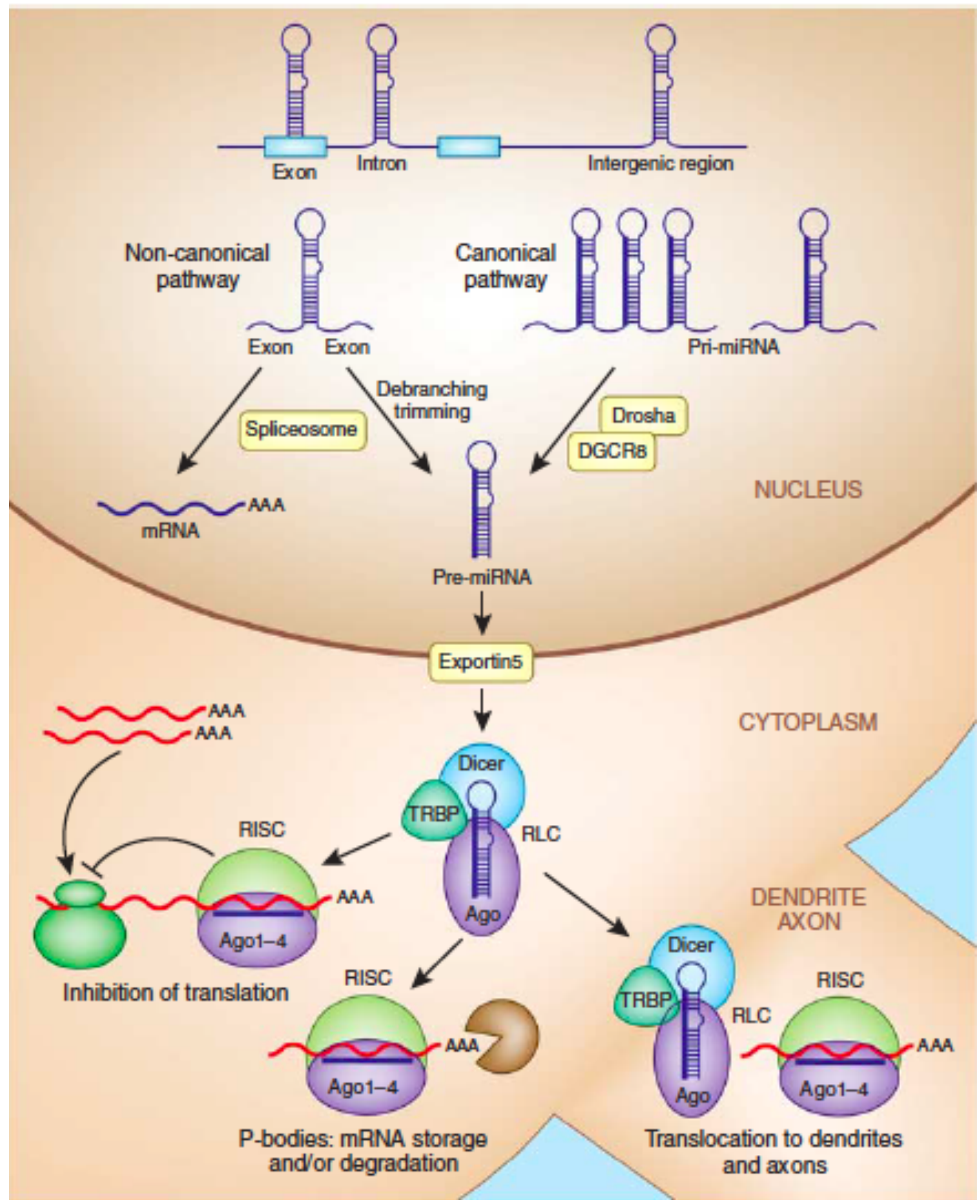

Figure 1 Biosynthesis of miRNAs picture adapted from (O'Carroll and Schaefer 2013) 


\section{References}

Aaltonen J, Laitinen MP, Vuojolainen K, Jaatinen R, Horelli-Kuitunen N, Seppa L, Louhio H, Tuuri T, Sjoberg J \& Butzow R. 1999. Human Growth Differentiation Factor 9 (GDF-9) and Its Novel Homolog GDF-9B Are Expressed in Oocytes during Early Folliculogenesis 18 , pp. 2744-2750.

Ajduk A, Małagocki A \& Maleszewski M 2008 Cytoplasmic maturation of mammalian oocytes: development of a mechanism responsible for sperminduced Ca2+ oscillations. Reproductive biology 8 3-22.

Akiyama T, Nagata M \& Aoki F 2006 Inadequate histone deacetylation during oocyte meiosis causes aneuploidy and embryo death in mice. Proceedings of the National Academy of Sciences 103 7339-7344.

Alizadeh Z, Kageyama S \& Aoki F 2005 Degradation of maternal mRNA in mouse embryos: selective degradation of specific mRNAs after fertilization. Molecular reproduction and development 72 281-290.

Andrews S 2012 FastQC. A quality control tool for high throughput sequence data..[h ttp://www. bioinformatics. bbsrc. ac. uk/projects/fastqc/].

Assou S, Anahory T, Pantesco V, Le Carrour T, Pellestor F, Klein B, Reyftmann L, Dechaud H, De Vos J \& Hamamah S 2006 The human cumulus--oocyte complex gene-expression profile. Human reproduction (Oxford, England) 21 1705-1719.

Bartel DP 2004 MicroRNAs: genomics, biogenesis, mechanism, and function. Cell 116 281-297.

Bartel DP 2009 MicroRNAs: target recognition and regulatory functions. Cell 136 215-233.

Bazzini AA, Lee MT \& Giraldez AJ 2012 Ribosome profiling shows that miR-430 reduces translation before causing mRNA decay in zebrafish. Science (New York, NY) 336 233-237.

Berezikov E, Chung W-J, Willis J, Cuppen E \& Lai EC 2007 Mammalian mirtron genes. Molecular cell 28 328-336.

Bettegowda A, Lee KB \& Smith GW 2008 Cytoplasmic and nuclear determinants of the maternal-to-embryonic transition. Reprod Fertil Dev 20 45-53.

Betthauser JM, Pfister-Genskow M, Xu H, Golueke PJ, Lacson JC, Koppang RW, Myers C, Liu B, Hoeschele I, Eilertsen KJ \& Leno GH 2006 Nucleoplasmin facilitates reprogramming and in vivo development of bovine nuclear transfer embryos. Mol Reprod Dev 73 977-986.

Bilezikjian LM, Blount AL, Donaldson CJ \& Vale WW 2006 Pituitary actions of ligands of the TGF-\{beta\} family: activins and inhibins. Reproduction 132 207.

Bodensteiner KJ, Clay CM, Moeller CL \& Sawyer HR 1999 Molecular Cloning of the Ovine Growth/Differentiation Factor-9 Gene and Expression of Growth/Differentiation Factor-9 in Ovine and Bovine Ovaries 1. Biology of reproduction 60 381-386.

Bohnsack MT, Czaplinski K \& GÖRLICH D 2004 Exportin 5 is a RanGTP-dependent dsRNA-binding protein that mediates nuclear export of pre-miRNAs. Rna $\mathbf{1 0}$ 185-191. 
Borchert GM, Lanier W \& Davidson BL 2006 RNA polymerase III transcribes human microRNAs. Nature structural \& molecular biology 13 1097-1101.

Borgel J, Guibert S, Li Y, Chiba H, Schübeler D, Sasaki H, Forné T \& Weber M 2010 Targets and dynamics of promoter DNA methylation during early mouse development. Nature Genetics 42 1093-1100.

Brodersen P \& Voinnet 02009 Revisiting the principles of microRNA target recognition and mode of action. Nature Reviews Molecular Cell Biology 10 141.

Burns K 2003 Roles of NPM2 in Chromatin and Nucleolar Organization in Oocytes and Embryos. Science (New York, NY) 300 633-636.

Burns KH, Viveiros MM, Ren Y, Wang P, DeMayo FJ, Frail DE, Eppig JJ \& Matzuk MM 2003 Roles of NPM2 in chromatin and nucleolar organization in oocytes and embryos. Science 300 633-636.

Cabot RA \& Prather RS 2003 Cleavage stage porcine embryos may have differing developmental requirements for karyopherins alpha2 and alpha3. Mol Reprod Dev 64 292-301.

Callaerts P, Vulsteke V, De Loof A \& Peumans W 1995 Lectin binding sites during Drosophila embryogenesis. Roux's archives of developmental biology 204 229-243.

Canastar A, Logel J, Graw S, Finlay-Schultz J, Osborne C, Palionyte M, Drebing C, Plehaty M, Wilson L, Eyeson R \& Leonard S 2012 Promoter Methylation and Tissue-Specific Transcription of the $\alpha 7$ Nicotinic Receptor Gene, CHRNA7. Journal of Molecular Neuroscience 47 389-400.

Carabatsos MJ, Elvin J, Matzuk MM \& Albertini DF 1998 Characterization of oocyte and follicle development in growth differentiation factor-9-deficient mice. Developmental Biology 204 373-384.

Chambers I, Colby D, Robertson M, Nichols J, Lee S, Tweedie S \& Smith A 2003 Functional expression cloning of Nanog, a pluripotency sustaining factor in embryonic stem cells. Cell 113 643-655.

Chen F, Cooney AJ, Wang Y, Law SW \& O'Malley BW 1994 Cloning of a novel orphan receptor (GCNF) expressed during germ cell development. Molecular endocrinology (Baltimore, Md.) 8 1434-1444.

Chendrimada TP, Gregory RI, Kumaraswamy E, Norman J, Cooch N, Nishikura K \& Shiekhattar R 2005 TRBP recruits the Dicer complex to Ago2 for microRNA processing and gene silencing. Nature 436 740-744.

Choi Y, Yuan D \& Rajkovic A 2008 Germ cell-specific transcriptional regulator sohlh2 is essential for early mouse folliculogenesis and oocyte-specific gene expression. Biology of reproduction 79 1176-1182.

Chook YM \& Blobel G 2001 Karyopherins and nuclear import. Current opinion in structural biology 11 703-715.

Corcoran DL, Pandit KV, Gordon B, Bhattacharjee A, Kaminski N \& Benos PV 2009 Features of mammalian microRNA promoters emerge from polymerase II chromatin immunoprecipitation data. PLOS ONE 4 e5279.

Coskun S \& Lin YC 1994 Effects of transforming growth factors and activin-A on in vitro porcine oocyte maturation. Molecular Reproduction and Development 38. 
Coy P, Romar R, Payton RR, McCann L, Saxton AM \& Edwards JL 2005 Maintenance of meiotic arrest in bovine oocytes using the S-enantiomer of roscovitine: effects on maturation, fertilization and subsequent embryo development in vitro. Reproduction 129 19-26.

da Silveira JC, Veeramachaneni DR, Winger QA, Carnevale EM \& Bouma GJ 2012 Cell-secreted vesicles in equine ovarian follicular fluid contain miRNAs and proteins: a possible new form of cell communication within the ovarian follicle. Biology of reproduction 8671.

Dammer EB \& Sewer MB 2008 Phosphorylation of CtBP1 by cAMP-dependent protein kinase modulates induction of CYP17 by stimulating partnering of CtBP1 and 2. Journal of Biological Chemistry 283 6925-6934.

Davis BN \& Hata A 2009 Regulation of MicroRNA Biogenesis: A miRiad of mechanisms. Cell Commun Signal 7 10.1186.

De La Fuente R, Viveiros MM, Burns KH, Adashi EY, Matzuk MM \& Eppig JJ 2004 Major chromatin remodeling in the germinal vesicle (GV) of mammalian oocytes is dispensable for global transcriptional silencing but required for centromeric heterochromatin function. Dev Biol 275 447-458.

Dingwall C \& Laskey RA 1991 Nuclear targeting sequences--a consensus. Trends Biochem Sci 16 478-481.

Diskin M, Parr M \& Morris D 2011 Embryo death in cattle: an update. Reproduction, Fertility and Development 24 244-251.

Dobbs KB, Spollen WG, Springer G \& Prather RS 2010 The role of cytoplasmic polyadenylation element sequence on mRNA abundance during porcine embryogenesis and parthenogenetic development. Molecular reproduction and development 77 699-709.

Dong J, Albertini DF, Nishimori K, Kumar TR, Lu N \& Matzuk MM 1996a Growth differentiation factor-9 is required during early ovarian folliculogenesis.

Dong J, Albertini DF, Nishimori K, Kumar TR, Lu N \& Matzuk MM 1996b Growth differentiation factor-9 is required during early ovarian folliculogenesis. Nature 383 531-535.

Dorey K \& Amaya E 2010 FGF signalling: diverse roles during early vertebrate embryogenesis. Development 137 3731-3742.

Driver AM, Peñagaricano F, Huang W, Ahmad KR, Hackbart KS, Wiltbank MC \& Khatib H 2012 RNA-Seq analysis uncovers transcriptomic variations between morphologically similar in vivo- and in vitro-derived bovine blastocysts. BMC Genomics 13118.

Dube JL, Wang P, Elvin J, Lyons KM, Celeste AJ \& Matzuk MM 1998 The bone morphogenetic protein 15 gene is X-linked and expressed in oocytes. Molecular Endocrinology 12 1809-1817.

Elvin JA, Clark AT, Wang P, Wolfman NM \& Matzuk MM 1999a Paracrine actions of growth differentiation factor-9 in the mammalian ovary. Molecular Endocrinology 13 1035-1048.

Elvin JA, Yan C, Wang P, Nishimori K \& Matzuk MM 1999b Molecular characterization of the follicle defects in the growth differentiation factor 9deficient ovary. Molecular Endocrinology 13 1018-1034. 
Eppig JJ 1996 Coordination of nuclear and cytoplasmic oocyte maturation in eutherian mammals. Reproduction, Fertility and Development 8 485-489.

Evans AC \& Walsh SW 2011 The physiology of multifactorial problems limiting the establishment of pregnancy in dairy cattle. Reproduction, Fertility and Development 24 233-237.

Feng P, Catt KJ \& Knecht M 1988 Transforming growth factor-beta stimulates meiotic maturation of the rat oocyte. Endocrinology 122181.

Fiedler SD, Carletti MZ, Hong X \& Christenson LK 2008 Hormonal regulation of MicroRNA expression in periovulatory mouse mural granulosa cells. Biology of reproduction 79 1030-1037.

Finn RD, Tate J, Mistry J, Coggill PC, Sammut SJ, Hotz HR, Ceric G, Forslund K, Eddy SR, Sonnhammer EL \& Bateman A 2008 The Pfam protein families database. Nucleic Acids Res 36 D281-288.

Geles KG \& Adam SA 2001 Germline and developmental roles of the nuclear transport factor importin alpha3 in C. elegans. Development 128 1817-1830.

Giraldez AJ, Mishima Y, Rihel J, Grocock RJ, Van Dongen S, Inoue K, Enright AJ \& Schier AF 2006 Zebrafish MiR-430 promotes deadenylation and clearance of maternal mRNAs. Science (New York, NY) 312 75-79.

Glazov EA, Kongsuwan K, Assavalapsakul W, Horwood PF, Mitter N \& Mahony TJ 2009 Repertoire of bovine miRNA and miRNA-like small regulatory RNAs expressed upon viral infection. PLoS ONE 4 e6349.

Goissis MD \& Cibelli JB 2014 Functional Characterization of SOX2 in Bovine Preimplantation Embryos. Biology of reproduction 9030.

Goossens K, Mestdagh P, Lefever S, Van Poucke M, Van Zeveren A, Van Soom A, Vandesompele J \& Peelman L 2013 Regulatory microRNA network identification in bovine blastocyst development. Stem cells and development 22 1907-1920.

Gu P, LeMenuet D, Chung AC-K, Mancini M, Wheeler DA \& Cooney AJ 2005 Orphan nuclear receptor GCNF is required for the repression of pluripotency genes during retinoic acid-induced embryonic stem cell differentiation. Molecular and Cellular Biology 25 8507-8519.

Gu P, Reid J, Gao X, Shaw C, Creighton C, Tran P, Zhou X, Drabek R, Steffen D, Hoang D, Weiss M, Naghavi A, El-Daye J, Khan M, Legge G, Wheeler D, Gibbs R, Miller J, Cooney AJ \& Gunaratne P 2008 Novel microRNA candidates and miRNA-mRNA pairs in embryonic stem (ES) cells. PLoS ONE 3 e2548.

Guiguen Y, Baroiller JF, Ricordel MJ, Iseki K, McMeel OM, Martin SA \& Fostier A 1999 Involvement of estrogens in the process of sex differentiation in two fish species: the rainbow trout (Oncorhynchus mykiss) and a tilapia (Oreochromis niloticus). Molecular reproduction and development 54 154162.

Hall MN, Griffin CA, Simionescu A, Corbett AH \& Pavlath GK 2011 Distinct roles for classical nuclear import receptors in the growth of multinucleated muscle cells. Developmental biology 357 248-258. 
Hammoud S, Emery BR, Dunn D, Weiss RB \& Carrell DT 2009 Sequence alterations in the YBX2 gene are associated with male factor infertility. Fertility and Sterility 91 1090-1095.

Han L, Witmer PDW, Casey E, Valle D \& Sukumar S 2007 DNA methylation regulates MicroRNA expression. Cancer Biology and Therapy 61284.

Hardie LJ, Laing KJ, Daniels GD, Grabowski PS, Cunningham C \& Secombes CJ 1998 Isolation of the first piscine transforming growth factor gene: analysis reveals tissue specific expression and a potential regulatory sequence in rainbow trout (Oncorhynchus mykiss). Cytokine 10 555-563.

Harms CA, Kennedy-Stoskopf S, Horne WA, Fuller FJ \& Tompkins WAF 2000 Cloning and sequencing hybrid striped bass (Morone saxatilis $\mathrm{x}$ M. chrysops) transforming growth factor- (TGF- ), and development of a reverse transcription quantitative competitive polymerase chain reaction (RTqcPCR) assay to measure TGF- mRNA of teleost fish. Fish and Shellfish Immunology 10 61-85.

Hassig CA, Tong JK, Fleischer TC, Owa T, Grable PG, Ayer DE \& Schreiber SL 1998 A role for histone deacetylase activity in HDAC1-mediated transcriptional repression. Proceedings of the National Academy of Sciences 95 3519-3524.

Hattori N, Nishino K, Ko Y, Hattori N, Ohgane J, Tanaka S \& Shiota K 2004 Epigenetic control of mouse Oct-4 gene expression in embryonic stem cells and trophoblast stem cells. Journal of Biological Chemistry 279 17063-17069.

Heldin CH, Miyazono K \& Ten Dijke P 1997 TGF- signalling from cell membrane to nucleus through SMAD proteins. NATURE-LONDON-465-471.

Hogarth CA, Calanni S, Jans DA \& Loveland KL 2006 Importin alpha mRNAs have distinct expression profiles during spermatogenesis. Dev Dyn 235 253-262.

Hood JK \& Silver PA 1998 Cse1p is required for export of Srp1p/importin-alpha from the nucleus in Saccharomyces cerevisiae.J Biol Chem 273 35142-35146.

Hossain M, Salilew-Wondim D, Schellander K \& Tesfaye D 2012 The role of microRNAs in mammalian oocytes and embryos. Animal reproduction science 134 36-44.

Hu J, Wang F, Yuan Y, Zhu X, Wang Y, Zhang Y, Kou Z, Wang S \& Gao S 2010a Novel importin-alpha family member Kpna7 is required for normal fertility and fecundity in the mouse. Journal of Biological Chemistry 285 3311333122.

Hu W, Gauthier L, Baibakov B, Jimenez-Movilla M \& Dean J 2010b FIGLA, a bHLH transcription factor, balances sexually dimorphic gene expression in postnatal oocytes. Molecular and Cellular Biology.

Irizarry RA, Ladd-Acosta C, Wen B, Wu Z, Montano C, Onyango P, Cui H, Gabo K, Rongione M \& Webster M 2009 Genome-wide methylation analysis of human colon cancer reveals similar hypo-and hypermethylation at conserved tissue-specific CpG island shores. Nature Genetics 41178.

Itman C, Miyamoto Y, Young J, Jans D \& Loveland K. 2009. Nucleocytoplasmic transport as a driver of mammalian gametogenesis. In Seminars in cell \& developmental biology 5, pp. 607-619. 
Jones PA 1985 Effects of 5-azacytidine and its 2' -deoxyderivative on cell differentiation and DNA methylation. Pharmacology \& therapeutics 28 17-27.

Jones PA 2012 Functions of DNA methylation: islands, start sites, gene bodies and beyond. Nature reviews Genetics 13 484-492.

Joshi S, Davies H, Sims LP, Levy SE \& Dean J 2007 Ovarian gene expression in the absence of FIGLA, an oocyte-specific transcription factor. BMC Developmental Biology 767.

Jüttermann R, Li E \& Jaenisch R 1994 Toxicity of 5-aza-2'-deoxycytidine to mammalian cells is mediated primarily by covalent trapping of DNA methyltransferase rather than DNA demethylation. Proceedings of the National Academy of Sciences 91 11797-11801.

Kass SU, Landsberger N \& Wolffe AP 1997 DNA methylation directs a timedependent repression of transcription initiation. Current Biology 7 157-165.

Kelley JB, Talley AM, Spencer A, Gioeli D \& Paschal BM 2010 Karyopherin alpha7 (KPNA7), a divergent member of the importin alpha family of nuclear import receptors. BMC Cell Biology 1163.

Khvorova A, Reynolds A \& Jayasena SD 2003 Functional siRNAs and miRNAs exhibit strand bias. Cell 115 209-216.

Kobayashi H, Sakurai T, Imai M, Takahashi N, Fukuda A, Yayoi O, Sato S, Nakabayashi K, Hata K \& Sotomaru Y 2012 Contribution of intragenic DNA methylation in mouse gametic DNA methylomes to establish oocyte-specific heritable marks. PLoS Genetics 8 e1002440.

Kocabas AM, Crosby J, Ross PJ, Otu HH, Beyhan Z, Can H, Tam WL, Rosa GJ, Halgren RG, Lim B, Fernandez E \& Cibelli JB 2006 The transcriptome of human oocytes. Proceedings of The National Academy Of Sciences Of The United States Of America 103 14027-14032.

Kosugi S, Hasebe M, Matsumura N, Takashima H, Miyamoto-Sato E, Tomita M \& Yanagawa H 2009a Six classes of nuclear localization signals specific to different binding grooves of importin alpha. The Journal of biological chemistry 284 478-485.

Kosugi S, Hasebe M, Tomita M \& Yanagawa H 2009b Systematic identification of cell cycle-dependent yeast nucleocytoplasmic shuttling proteins by prediction of composite motifs. Proceedings of the National Academy of Sciences 106 10171-10176.

Kurosaka S, Eckardt S \& McLaughlin KJ 2004 Pluripotent lineage definition in bovine embryos by Oct4 transcript localization. Biol Reprod 71 1578-1582.

Laing KJ, Cunningham C \& Secombes CJ 2000 Genes for three different isoforms of transforming growth factor- are present in plaice (Pleuronectes platessa) DNA. Fish and Shellfish Immunology 10 261-271.

Laitinen M, Vuojolainen K, Jaatinen R, Ketola I, Aaltonen J, Lehtonen E, Heikinheimo M \& Ritvos 01998 A novel growth differentiation factor-9 (GDF-9) related factor is co-expressed with GDF-9 in mouse oocytes during folliculogenesis. Mechanisms of development 78 135-140. 
Lam YW \& Ng TB 2002 Purification and characterization of a rhamnose-binding lectin with immunoenhancing activity from grass carp (Ctenopharyngodon idellus) ovaries. Protein expression and purification 26 378-385.

Lan J, Hua S, Zhang H, Song Y, Liu J \& Zhang Y 2010 Methylation patterns in 5 'terminal regions of pluripotency-related genes in bovine in vitro fertilized and cloned embryos. Journal of Genetics and Genomics 37 297-304.

Lan ZJ, Gu P, Xu X, Jackson KJ, DeMayo FJ, O'Malley BW \& Cooney AJ 2003 GCNF - dependent repression of BMP - 15 and GDF - 9 mediates gamete regulation of female fertility. The EMBO Journal 22 4070-4081.

Lange A, Mills RE, Lange CJ, Stewart M, Devine SE \& Corbett AH 2007 Classical nuclear localization signals: definition, function, and interaction with importin alpha. J Biol Chem 282 5101-5105.

Langmead B \& Salzberg SL 2012 Fast gapped-read alignment with Bowtie 2. Nature methods 9 357-359.

Lee K-B, Wee G, Zhang K, Folger JK, Knott JG \& Smith GW 2014 Functional Role of the Bovine Oocyte-Specific Protein JY-1 in Meiotic Maturation, Cumulus Expansion, and Subsequent Embryonic Development. Biology of reproduction.

Lee RC, Feinbaum RL \& Ambros V 1993 The C. elegans heterochronic gene $<$ i $>$ lin$4</ \mathrm{i}>$ encodes small RNAs with antisense complementarity to $<\mathrm{i}>\operatorname{lin}-14</ \mathrm{i}>$. Cell 75 843-854.

Li L, Zheng P \& Dean J 2010 Maternal control of early mouse development. Development 137 859-870.

Liang L, Soyal SM \& Dean J 1997 FIGalpha, a germ cell specific transcription factor involved in the coordinate expression of the zona pellucida genes. Development 124 4939-4947.

Lingenfelter BM, Tripurani SK, Tejomurtula J, Smith GW \& Yao J 2011 Molecular cloning and expression of bovine nucleoplasmin 2 (NPM2): a maternal effect gene regulated by miR-181a. Reprod Biol Endocrinol 940.

Lund E, Liu M, Hartley RS, Sheets MD \& Dahlberg JE 2009 Deadenylation of maternal mRNAs mediated by miR-427 in Xenopus laevis embryos. RNA (New York, NY) 15 2351-2363.

Ma J, Flemr M, Stein P, Berninger P, Malik R, Zavolan M, Svoboda P \& Schultz RM 2010 MicroRNA activity is suppressed in mouse oocytes. Current biology: CB 20 265-270.

Maatouk DM, Kellam LD, Mann MRW, Lei H, Li E, Bartolomei MS \& Resnick JL 2006 DNA methylation is a primary mechanism for silencing postmigratory primordial germ cell genes in both germ cell and somatic cell lineages. Development 133 3411-3418.

Maeda C, Sato S, Hattori N, Tanaka S, Yagi S \& Shiota K 2008 DNA hypomethylation circuit of the mouse oocyte-specific histone H1foo gene in female germ cell lineage. Biology of reproduction 78 816-821.

Mandke P, Wyatt N, Fraser J, Bates B, Berberich SJ \& Markey MP 2012 MicroRNA-34a Modulates MDM4 Expression via a Target Site in the Open Reading Frame. PLoS ONE 7 e42034. 
Mason DA, Fleming RJ \& Goldfarb DS 2002 Drosophila melanogaster importin $\alpha 1$ and $\alpha 3$ can replace importin $\alpha 2$ during spermatogenesis but not oogenesis. Genetics 161 157-170.

Massague J 1998 TGF- signal transduction. Annual review of biochemistry 67 753791.

Mathe E, Bates H, Huikeshoven H, Deak P, Glover DM \& Cotterill S 2000 Importin-alpha3 is required at multiple stages of Drosophila development and has a role in the completion of oogenesis. Dev Biol 223 307-322.

Matzuk MM, Burns KH, Viveiros MM \& Eppig JJ. 2002. Intercellular communication in the mammalian ovary: oocytes carry the conversation 5576, pp. 2178-2180.

Memili E \& First NL 2000 Zygotic and embryonic gene expression in cow: a review of timing and mechanisms of early gene expression as compared with other species. Zygote 8 87-96.

Messerschmidt DM. 2012. Should I stay or should I go: Protection and maintenance of DNA methylation at imprinted genes. In Epigenetics 9.

Miyara F, Migne C, Dumont - Hassan M, Meur AL, Cohen - Bacrie P, Aubriot FX, Glissant A, Nathan C, Douard S \& Stanovici A 2003 Chromatin configuration and transcriptional control in human and mouse oocytes. Molecular reproduction and development 64 458-470.

Moore K \& Thatcher WW 2006 Major advances associated with reproduction in dairy cattle. Journal of dairy science 89 1254-1266.

Mulheron GW, Danielpour D \& Schomberg DW 1991 Rat thecal/interstitial cells express transforming growth factor-beta type 1 and 2, but only type 2 is regulated by gonadotropin in vitro. Endocrinology(Philadelphia) 129 368374.

Nagahama Y \& Yamashita M 2008 Regulation of oocyte maturation in fish. Development, Growth \& Differentiation 50 195-219.

Nishino K, Hattori N, Tanaka S \& Shiota K 2004 DNA methylation-mediated control of Sry gene expression in mouse gonadal development. Journal of Biological Chemistry 279 22306-22313.

Niu C, Zhang J, Gao F, Yang L, Jia M, Zhu H \& Gong W 2012 FUS-NLS/transportin 1 complex structure provides insights into the nuclear targeting mechanism of FUS and the implications in ALS. PLOS ONE 7 e47056.

O'Carroll D \& Schaefer A 2013 General principals of miRNA biogenesis and regulation in the brain. Neuropsychopharmacology 38 39-54.

Otis KO, Thompson KR \& Martin KC 2006 Importin-mediated nuclear transport in neurons. Current opinion in neurobiology 16 329-335.

Otsuka M, Zheng M, Hayashi M, Lee J-D, Yoshino O, Lin S \& Han J 2008 Impaired microRNA processing causes corpus luteum insufficiency and infertility in mice. The Journal of Clinical Investigation 1181944.

Ozawa M, Sakatani M, Yao J, Shanker S, Yu F, Yamashita R, Wakabayashi S, Nakai K, Dobbs KB, Sudano MJ, Farmerie WG \& Hansen PJ 2012 Global gene expression of the inner cell mass and trophectoderm of the bovine blastocyst. BMC Developmental Biology 12 1-1. 
Pangas SA \& Rajkovic A 2006 Transcriptional regulation of early oogenesis: in search of masters. Hum Reprod Update 12 65-76.

Panning B \& Jaenisch R 1996 DNA hypomethylation can activate Xist expression and silence X-linked genes. Genes \& Development 10 1991-2002.

Paynton BV, Rempel R \& Bachvarova R 1988 Changes in state of adenylation and time course of degradation of maternal mRNAs during oocyte maturation and early embryonic development in the mouse. Developmental biology 129 304-314.

Philpott A, Leno GH \& Laskey RA 1991 Sperm decondensation in Xenopus egg cytoplasm is mediated by nucleoplasmin. Cell 65 569-578.

Place RF, Li L, Pookot D, Noonan EJ \& Dahiya R 2008 MicroRNA-373 induces expression of genes with complementary promoter sequences. Proceedings Of The National Academy Of Sciences Of The United States Of America 105 1608-1613.

Properzi F, Logozzi M \& Fais S 2013 Exosomes: the future of biomarkers in medicine. Biomarkers in medicine 7 769-778.

Qiu GF, Weber GM, Rexroad CE \& Yao J 2008 Identification of RtGST-1, a novel germ cell-specific mRNA-like transcript predominantly expressed in early previtellogenic oocytes in rainbow trout (Oncorhynchus mykiss). Molecular reproduction and development 75 723-730.

Rajkovic A 2004 NOBOX Deficiency Disrupts Early Folliculogenesis and OocyteSpecific Gene Expression. Science 305 1157-1159.

Rajkovic A, Pangas SA, Ballow D, Suzumori N \& Matzuk MM 2004 NOBOX deficiency disrupts early folliculogenesis and oocyte-specific gene expression. Science 305 1157-1159.

Ramachandra RK, Lankford SE, Weber GM, Rexroad CE \& Yao J 2007 Identification of OORP-T, a novel oocyte-specific gene encoding a protein with a conserved oxysterol binding protein domain in rainbow trout. Molecular reproduction and development 74 502-511.

Ramachandra RK, Salem M, Gahr S, Rexroad CE \& Yao J 2008 Cloning and characterization of microRNAs from rainbow trout (Oncorhynchus mykiss): their expression during early embryonic development. BMC Dev Biol 841.

Rao X, Evans J, Chae H, Pilrose J, Kim S, Yan P, Huang R, Lai H, Lin H \& Liu Y 2013 CpG island shore methylation regulates caveolin-1 expression in breast cancer. Oncogene 32 4519-4528.

Rice JC \& Allis CD 2001 Histone methylation versus histone acetylation: new insights into epigenetic regulation. Current opinion in cell biology 13 263-273.

Richards JAS. 2007. Genetics of ovulation. In Seminars in Reproductive Medicine 04, pp. 235-242.

Rodda D 2005 Transcriptional Regulation of Nanog by OCT4 and SOX2. The Journal of biological chemistry $28024731-24737$.

Rodriguez A, Griffiths-Jones S, Ashurst JL \& Bradley A 2004 Identification of mammalian microRNA host genes and transcription units. Genome research 14 1902-1910. 
Rodriguez K \& Farin C 2004 Developmental capacity of bovine cumulus oocyte complexes after transcriptional inhibition of germinal vesicle breakdown. Theriogenology 61 1499-1511.

Rodriguez KF \& Farin CE 2003 Gene transcription and regulation of oocyte maturation. Reproduction, Fertility and Development 16 55-67.

Rousseau C, Felin M, Doyennette - Moyne MA \& Sève AP 1997 CBP70, a glycosylated nuclear lectin. Journal of cellular biochemistry 66 370-385.

Ruddock-D'Cruz NT, Hall VJ, Tecirlioglu RT \& French AJ 2007 Gene expression analysis of single preimplantation bovine embryos and the consequence for developmental potential. Soc Reprod Fertil Suppl 64 341-363.

Russell DL \& Robker RL 2007 Molecular mechanisms of ovulation: co-ordination through the cumulus complex. Human Reproduction Update 13 289-312.

Sagata N, Watanabe N, Woude GFV \& Ikawa Y 1989 The c-mos proto-oncogene product is a cytostatic factor responsible for meiotic arrest in vertebrate eggs.

Sang Q, Yao Z, Wang H, Feng R, Wang H, Zhao X, Xing Q, Jin L, He L, Wu L \& Wang L 2013 Identification of microRNAs in human follicular fluid: characterization of microRNAs that govern steroidogenesis in vitro and are associated with polycystic ovary syndrome in vivo. The Journal of Clinical Endocrinology \& Metabolism 98 3068-3079.

Santos F, Hendrich B, Reik W \& Dean W 2002 Dynamic reprogramming of DNA methylation in the early mouse embryo. Developmental biology 241 172-182.

SchmidP CD \& vanderPuttenH M 1994 BilbeG: Expression of TGF-beta s and TGFbeta type II receptor mRNAs in mouse folliculogenesis: Stored maternal TGFbeta 2 message in oocytes. Biochem Biophys Res Commun 201 649-656.

Schwoebel ED, Talcott B, Cushman I \& Moore MS 1998 Ran-dependent signalmediated nuclear import does not require GTP hydrolysis by Ran. The Journal of biological chemistry 273 35170-35175.

Sendai Y, Itoh T, Yamashita S \& Hoshi H 2001 Molecular cloning of a cDNA encoding a bovine growth differentiation factor-9 (GDF-9) and expression of GDF-9 in bovine ovarian oocytes and in vitro-produced embryos. Cloning 3 310.

Shiina N, Tateno H, Ogawa T, Muramoto K, Saneyoshi M \& Kamiya H 2002 Isolation and characterization of L-rhamnose-binding lectins from chum salmon (Oncorhynchus keta) eggs. Fisheries Science 68 1352-1366.

Shiota K 2004 DNA methylation profiles of CpG islands for cellular differentiation and development in mammals. Cytogenetic and genome research 105 325334.

Shirai T, Watanabe Y, Lee M-s, Ogawa T \& Muramoto K 2009 Structure of rhamnose-binding lectin CSL3: unique pseudo-tetrameric architecture of a pattern recognition protein. Journal of molecular biology 391 390-403.

Singh B, Barbe GJ \& Armstrong DT 1993 Factors influencing resumption of meiotic maturation and cumulus expansion of porcine oocyte-cumulus cell complexes in vitro. Molecular Reproduction and Development 36. 
Smallwood SA, Tomizawa S, Krueger F, Ruf N, Carli N, Segonds-Pichon A, Sato S, Hata K, Andrews SR \& Kelsey G 2011 Dynamic CpG island methylation landscape in oocytes and preimplantation embryos. Nature Publishing Group 43 811-814.

Sohel MM, Hoelker M, Noferesti S, Salilew-Wondim D, Tholen E, Looft C, Rings F, Uddin M, Spencer T, Schellander K \& Tesfaye D 2013 Exosomal and Non-Exosomal Transport of Extra-Cellular microRNAs in Follicular Fluid: Implications for Bovine Oocyte Developmental Competence. PLOS ONE 8 e78505.

Song F, Mahmood S, Ghosh S, Liang P, Smiraglia DJ, Nagase H \& Held WA 2009 Tissue specific differentially methylated regions (TDMR): Changes in DNA methylation during development. Genomics 93 130-139.

Soyal S, Amleh A \& Dean J 2000a FIGalpha, a germ cell-specific transcription factor required for ovarian follicle formation. Development 1274645.

Soyal SM, Amleh A \& Dean J 2000b FIGalpha, a germ cell-specific transcription factor required for ovarian follicle formation. Development 127 4645-4654.

Suh N \& et al. 2011 Small RNAs in early mammalian development: from gametes to gastrulation. Development1-9.

Sun Q, Liu K \& Kikuchi K 2008a Oocyte-Specific Knockout: A Novel In Vivo Approach for Studying Gene Functions During Folliculogenesis, Oocyte Maturation, Fertilization, and Embryogenesis. Biology of Reproduction 79 1014.

Sun Q, Liu K \& Kikuchi K 2008b Oocyte-Specific Knockout: A Novel In Vivo Approach for Studying Gene Functions During Folliculogenesis, Oocyte Maturation, Fertilization, and Embryogenesis. Biology of reproduction 79 1014.

Suzumori N, Yan C, Matzuk MM \& Rajkovic A 2002 Nobox is a homeoboxencoding gene preferentially expressed in primordial and growing oocytes. Mechanisms of development 111 137-141.

Swiech L, Kisiel K, Czolowska R, Zientarski M \& Borsuk E 2007 Accumulation and dynamics of proteins of the MCM family during mouse oogenesis and the first embryonic cell cycle. International Journal of Developmental Biology $\mathbf{5 1}$ 283.

Tateno H, Ogawa T, Muramoto K, Kamiya H, Hirai T \& Saneyoshi M 2001 A novel rhamnose-binding lectin family from eggs of steelhead trout (Oncorhynchus mykiss) with different structures and tissue distribution. Bioscience, biotechnology, and biochemistry 65 1328-1338.

Tateno H, Shibata Y, Nagahama Y, Hirai T, Saneyoshi M, Ogawa T, Muramoto K \& Kamiya H 2002a Tissue-specific expression of rhamnose-binding lectins in the steelhead trout (Oncorhynchus mykiss). Bioscience, biotechnology, and biochemistry 66 1427-1430.

Tateno H, Yamaguchi T, Ogawa T, Muramoto K, Watanabe T, Kamiya H \& Saneyoshi M 2002b Immunohistochemical localization of rhamnose-binding lectins in the steelhead trout (Oncorhynchus mykiss). Developmental and comparative immunology 26 543-550. 
Tay Y, Zhang J, Thomson AM, Lim B \& Rigoutsos I 2008 MicroRNAs to Nanog, Oct4 and Sox 2 coding regions modulate embryonic stem cell differentiation. Nature 455 1124-1128.

Tejomurtula J, Lee K, Tripurani SK, Smith GW \& Yao J 2009 Role of Importin Alpha8, a New Member of the Importin Alpha Family of Nuclear Transport Proteins, in Early Embryonic Development in Cattle. Biology of reproduction 81 333-342.

Thiagalingam S, CHENG KH, Lee HJ, Mineva N, Thiagalingam A \& Ponte JF 2003 Histone deacetylases: unique players in shaping the epigenetic histone code. Annals of the New York Academy of Sciences 983 84-100.

Trapnell C, Pachter L \& Salzberg SL 2009 TopHat: discovering splice junctions with RNA-Seq. Bioinformatics 25 1105-1111.

Tripurani SK, Lee K, Wang L, Wee G, Smith GW, Lee YS, Latham KE \& Yao J 2010a A Novel Functional Role for the Oocyte-Specific Transcription Factor Newborn Ovary Homeobox (NOBOX) during Early Embryonic Development in Cattle. Endocrinology.

Tripurani SK, Lee K, Wee G, Smith GW \& Yao J 2011 MicroRNA-196a regulates bovine newborn ovary homeobox gene (NOBOX) expression during early embryogenesis. BMC Developmental Biology 1125.

Tripurani SK, Wee G, Lee K, Smith GW, Wang L \& Jianboyao 2013 MicroRNA-212 post-transcriptionally regulates oocyte-specific basic-helix-loop-helix transcription factor, factor in the germline alpha (FIGLA), during bovine early embryogenesis. PLOS ONE 8 e76114.

Tripurani SK, Xiao C, Salem M \& Yao J 2010b Cloning and analysis of fetal ovary microRNAs in cattle. Animal reproduction science 120 16-22.

Valadi H, Ekström K, Bossios A, Sjöstrand M, Lee JJ \& Lötvall JO 2007 Exosomemediated transfer of mRNAs and microRNAs is a novel mechanism of genetic exchange between cells. Nature Cell Biology 9 654-659.

Van Soom A, Van Vlaenderen I, Mahmoudzadeh A, Deluyker H \& de Kruif A 1992 Compaction rate of in vitro fertilized bovine embryos related to the interval from insemination to first cleavage. Theriogenology 38 905-919.

Vasudevan S 2012 Posttranscriptional upregulation by microRNAs. Wiley interdisciplinary reviews RNA 3 311-330.

Virant-Klun I, Knez K, Tomazevic T \& Skutella T 2013 Gene expression profiling of human oocytes developed and matured in vivo or in vitro. BioMed research international 2013879489.

Vitt UA \& Hsueh AJW 2002 Stage-dependent role of growth differentiation factor-9 in ovarian follicle development. Molecular and cellular endocrinology 186 211-217.

Wang H, Wang X, Archer TK, Zwaka TP \& Cooney AJ 2014 GCNF-dependent activation of cyclin D1 expression via repression of Mir302a during ES cell differentiation. Stem Cells.

Wang L, Tripurani SK, Wanna W, Rexroad CE \& Yao J 2013a Cloning and characterization of a novel oocyte-specific gene encoding an F-Box protein in rainbow trout (Oncorhynchus mykiss). Reproductive Biology and Endocrinology 1186. 
Wang L, Yao J \& Smith GW. 2013b. MicroRNA-1296 Regulates Bovine Oocytespecific Karyrophrin alpha 7 Expression During Maternal-Zygotic Transition. In Society for Study of Reprocution 46th annual meeting 365.

Wang X, Park KE, Koser S, Liu S, Magnani L \& Cabot RA 2011 KPNA7, an oocyteand embryo-specific karyopherin $\alpha$ subtype, is required for porcine embryo development. Reproduction, Fertility and Development.

Wang Z, Gerstein M \& Snyder M 2009 RNA-Seq: a revolutionary tool for transcriptomics. Nature reviews Genetics 10 57-63.

Watanabe Y, Tateno H, Nakamura-Tsuruta S, Kominami J, Hirabayashi J, Nakamura 0, Watanabe T, Kamiya H, Naganuma T, Ogawa T, Naudé RJ \& Muramoto K 2009 The function of rhamnose-binding lectin in innate immunity by restricted binding to Gb3. Developmental and comparative immunology 33 187-197.

Weis K 2003 Regulating access to the genome: nucleocytoplasmic transport throughout the cell cycle. Cell 112 441-451.

Westholm JO \& Lai EC 2011 Mirtrons: microRNA biogenesis via splicing. Biochimie 93 1897-1904.

Winter J, Jung S, Keller S, Gregory RI \& Diederichs S 2009 Many roads to maturity: microRNA biogenesis pathways and their regulation. Nature Cell Biology 11 228-234.

Xie D, Chen C, Ptaszek L, Xiao S, Cao X, Fang F, Ng H, Lewin H, Cowan C \& Zhong S 2010 Rewirable gene regulatory networks in the preimplantation embryonic development of three mammalian species. Genome research $\mathbf{2 0}$ 804-815.

Yamaguchi S, Kimura H, Tada M, Nakatsuji N \& Tada T 2005 Nanog expression in mouse germ cell development. Gene Expression Patterns 5 639-646.

Yan C, Wang P, DeMayo J, DeMayo FJ, Elvin JA, Carino C, Prasad SV, Skinner SS, Dunbar BS \& Dube JL 2001 Synergistic roles of bone morphogenetic protein 15 and growth differentiation factor 9 in ovarian function. Molecular Endocrinology 15 854-866.

Yao G, Yin M, Lian J, Tian H, Liu L, Li X \& Sun F 2010a MicroRNA-224 is involved in transforming growth factor-beta-mediated mouse granulosa cell proliferation and granulosa cell function by targeting Smad4. Molecular endocrinology (Baltimore, Md) 24 540-551.

Yao N, Yang B-Q, Liu Y, Tan X-Y, Lu C-L, Yuan X-H \& Ma X 2010b Folliclestimulating hormone regulation of microRNA expression on progesterone production in cultured rat granulosa cells. Endocrine 38 158-166.

Yasuhara N, Shibazaki N, Tanaka S, Nagai M, Kamikawa Y, Oe S, Asally M, Kamachi Y, Kondoh H \& Yoneda Y 2007 Triggering neural differentiation of ES cells by subtype switching of importin-alpha. Nat Cell Biol 9 72-79.

Yasuhara N, Yamagishi R, Arai Y, Mehmood R, Kimoto C, Fujita T, Touma K, Kaneko A, Kamikawa Y \& Moriyama T 2013 Importin Alpha Subtypes Determine Differential Transcription Factor Localization in Embryonic Stem Cells Maintenance. Developmental cell 26 123-135. 
Young JC, Major AT, Miyamoto Y, Loveland KL \& Jans DA 2011 Distinct effects of importin $\alpha 2$ and $\alpha 4$ on 0ct3/4 localization and expression in mouse embryonic stem cells. The FASEB Journal 25 3958-3965.

Yu L-G, Fernig DG, White MR, Spiller DG, Appleton P, Evans RC, Grierson I, Smith JA, Davies H \& Gerasimenko OV 1999 Edible mushroom (Agaricus bisporus) lectin, which reversibly inhibits epithelial cell proliferation, blocks nuclear localization sequence-dependent nuclear protein import. Journal of Biological Chemistry 274 4890-4899.

Zhan Y \& Jimmy K 2000 Molecular isolation and characterisation of carp transforming growth factor 1 from activated leucocytes. Fish and Shellfish Immunology 10 309-318.

Zhang X, Wu M, Xiao H, Lee M, Levin L, Leung Y \& Ho S 2010 Methylation of a single intronic CpG mediates expression silencing of the PMP24 gene in prostate cancer. The Prostate $\mathbf{7 0}$ 765-776.

Zheng P \& Dean J 2007a Oocyte-specific genes affect folliculogenesis, fertilization, and early development. Seminars in Reproductive Medicine 25 243-251.

Zheng P \& Dean J 2007b Oocyte-specific genes affect folliculogenesis, fertilization, and early development. Semin Reprod Med 25 243-251. 


\section{Chapter 1:}

Kpna7 interacts with egg-specific nuclear factors in rainbow trout (Oncorhynchus mykiss)

Lei Wang, Hao Ma, Liyuan Fu and Jianbo Yao*

Division of Animal and Nutritional Sciences, West Virginia University, Morgantown, WV 26506, USA

Key words: importin, protein-protein interaction, nuclear transport, embryogenesis

Grant sponsor: USDA ARS Cooperative Agreement No. 58-1930-0-059

Conflict of interest: The authors have no conflict of interest to declare.

Abbreviation: KPNA, Karyopherin alpha; NLS, Nuclear localization signal; IBB, Importin beta binding domain; ARM, Armadillo domain; St13, Steel trout L-rhamnose-binding lectin 3; bp basepair; UTR, Untranslated region; ORF, Open reading frame; H3K27me3, Trimethylated histone H3 at lysine-27; HEK293, Human embryonic kidney 293 cell; EGFP, Enhanced green florescent protein.

*Corresponding author

Jianbo Yao

West Virginia University

Morgantown, WV 26506-6108

U.S.A.

Tel.: 304-293-1948

Fax: 304-293-2232

E-mail: jianbo.yao@mail.wvu.edu 


\begin{abstract}
Nuclear proteins are required for initiation of transcription in early embryos before embryonic genome activation. The regulation of transportation of nuclear proteins is mediated by transport factors known as importins (karyopherins). Kpna7 is a newly discovered member of the importin $\alpha$ family, which is critical for early development in mammals. In this study, we report the molecular characterization of rainbow trout Kpna7. The cDNA for rainbow trout kpna7 encodes a protein of 519 amino acids, which contains a conserved IBB (importin $\beta$ binding domain) domain and seven ARM (armadillo/betacatenin-like repeat) motifs. RT-PCR and Western blot analyses revealed that Kpna7 is specifically expressed in eggs/ovary. Real-time PCR analysis demonstrated that expression of kpna7 mRNA is high in unfertilized eggs and decreases gradually in early stage embryos until 3 days post fertilization followed by a sharp decrease reaching a level that is barely detectable in day 4 embryos and thereafter. Using a yeast two-hybrid screening system, we identified two Kpna7-interacting proteins from a rainbow trout egg cDNA library: St13 (rhamnose-binding lectin 3) and an uncharacterized protein. Both genes appear to be expressed specifically in eggs/testis as revealed by RT-PCR analysis. Co-immunoprecipitation assays confirmed the interaction between Kpna7 and St13, and co-transfection experiments using EGFP-tagged Stl3 showed that Kpna7 facilitates the nuclear transportation of Stl3 through interaction with the predicted NLS cluster at the Cterminus of Stl3. Our data suggest that Kpna7 may function as a unique nuclear transport receptor for egg-specific proteins important for early embryonic development.
\end{abstract}




\section{Introduction}

To fully understand the scope and features of transcriptional dynamics during early embryogenesis, it is essential to identify the regulatory elements important for directing the developmental processes. Passage through successive stages of embryonic development and differentiation cannot be achieved without the help of nuclear proteins such as transcription factors and chromatin-remodeling factors that act in the nucleus (Itman et al. 2009). Therefore, the transportation of such nuclear proteins into nucleus through nuclear pores is a vital event in the development of gametes and early embryos (Geles and Adam 2001; Mason et al. 2002; Tejomurtula et al. 2009). A family of nuclear transporters known as importins (also named karyopherins) are the major players in the translocation of macromolecules through an active, energy-dependent nuclear import system (Chook and Blobel 2001; Otis et al. 2006). The importin $\alpha$ and importin $\beta$ heterodimer import pathway is the best-characterized nuclear import system. Importin $\alpha$ binds to the nuclear localization signal (NLS) of a cargo protein and importin $\beta$ docks the protein complex to the nuclear pore followed by translocation into the nucleus. Once inside the nucleus, with the help of the RanGTPase system, the cargo protein is disassociated from importin $\alpha$ (Hood and Silver 1998; Schwoebel et al. 1998; Weis 2003). The NLSs recognized by importin $\alpha$ proteins are either a short stretch of basic amino acids or two basic stretches separated by a spacer of 10 to 37 amino acids (Dingwall and Laskey 1991; Kosugi et al. 2009a). Different importin $\alpha$ proteins possess distinct NLS-binding specificities, and developmental regulation of gene expression can 
be affected by changes in the expression of importin $\alpha$ genes. During mouse spermatogenesis, neural lineage specification, and maintenance of embryonic stem cells, shifts of importin $\alpha$ gene expression were observed, which were accompanied by the determination of cell fate (Hogarth et al. 2006; Yasuhara et al. 2006; Yasuhara et al. 2013; Young et al. 2011). These studies indicate that importins are important regulators of cellular differentiation.

To date, seven importin a family members (Kpna1, Kpna2, Kpna3, Kpna4, Kpna5, Kpna6 and Kpna7) have been characterized in mammals. Only Kpna7 is specifically expressed in oocytes and early embryos (Hu et al. 2010; Tejomurtula et al. 2009; Wang et al. 2011). During early embryogenesis, the expression of bovine and porcine Kpna7 is high in early stage embryos, but is barely detectable in morula and blastocyst stage embryos. Knockdown of Kpna7 leads to arrested embryonic development in cattle and swine (Tejomurtula et al. 2009; Wang et al. 2011). Knockout of Kpna7 in female mice causes fecundity reduction and sex imbalance, as well as down-regulation of histone H3K27me3 (Hu et al. 2010). Although the role of Kpna7 as a maternal effect factor critical for early development in mammals is well established, little is known about the essential nuclear proteins transported by Kpna7.

Rainbow trout is one of the major fish species cultivated widely in the world. It has been utilized as a model species in many research areas (Thorgaard et al. 2002). The focus of our research is on the understanding of the functional contribution of key egg-specific genes to early embryonic development in rainbow trout. In this study, we report the characterization of Kpna7 in rainbow trout, and show that rainbow trout Kpna7 interacts with Stl3, an egg-specific nuclear protein. Results support our hypothesis that Kpna7 
mediates developmental processes through transporting important egg-specific nuclear factors.

\section{Results}

Characterization of rainbow trout kpna7 cDNA sequence

Using bovine kpna7 cDNA sequence, we searched the Dana Farber Cancer Institute (DFCI) Gene Indices database and identified a TC sequence (TC149065) containing a partial sequence for rainbow trout kpna7. Multiple EST sequences that were used to generate the TC sequence were identified from the database. The longest cDNA clone was retrieved from an arrayed rainbow trout egg cDNA library and the insert of the clone was completely sequenced. The cDNA sequence for rainbow trout kpna7 gene is 1,787 bp in length, which has been deposited in the GenBank database with the accession number: KJ625239. Analysis of the kpna7 cDNA sequence showed that it contains a 145 bp 5'-untranslated region (5'UTR), an open reading frame (ORF) of 1,560 bp and a 3'untranslated region (3'UTR) of 82 bp. A typical polyadenylation signal sequence (AATAAA) and a cytoplasmic polyadenylation element (TTAAAAT) were identified in the 3'UTR. The ORF of the cDNA encodes a protein of 519 amino acids with a predicted molecular weight of $57 \mathrm{kDa}$. The protein is predicted to contain a conserved IBB (Importin $\beta$ binding domain) domain at the $\mathrm{N}$-terminus and seven ARM (Armadillo/betacatenin-like repeat) motifs in the central region. Comparison of amino acid sequence identity between rainbow trout Kpna7 and the orthologs of other species revealed that rainbow trout Kpna7 shares $75.5 \%, 66.4 \%, 54.5 \%$ and $52.7 \%$ sequence identity with zebrafish, Xenopus, human and bovine Kpna7, respectively. Phylogenetic analysis of the 
rainbow trout Kpna proteins revealed that Kpna7 is more closely related to Kpna2 (Supplementary Figure S1), which agrees with the results of previous studies (Kelley et al. 2010; Tejomurtula et al. 2009).

Expression analysis of kpna7 mRNA and protein

Analysis of tissue distribution of kpna7 mRNA by RT-PCR revealed that rainbow trout kpna7 is predominantly expressed in eggs (Fig. 1A). Minor expression of kpna7 mRNA was observed in testis but not in any other somatic tissues. In contrast, other importin $\alpha$ genes including kpna2, kpna2-like, kpna4 and kpna5 are ubiquitously expressed in the tissues examined (Fig. 1B). Analysis of kpna7 mRNA expression during ovarian development revealed that kpna7 is expressed in ovaries of various developmental stages with significantly higher expression in ovaries at early vitellogenic stage (Fig. 1C). The expression profile of kpna7 mRNA during embryonic development in rainbow trout was analyzed by quantitative real-time PCR using cDNAs derived from unfertilized eggs and embryos of different developmental stages. As shown in Fig. 1D, the expression of kpna7 mRNA is high in unfertilized eggs and decreases gradually in early stage embryos until 3 days post fertilization (3d) followed by a sharp decrease reaching a level that is barely detectable in $4 \mathrm{~d}$ embryos and thereafter.

Western blot analysis using a polyclonal antibody raised in rabbit against a synthetic peptide of Kpna7 protein showed that Kpna7 protein can only be detected in the ovary but not in other somatic tissues including gill, heart, kidney, liver, spleen and stomach (Fig. 2). Localization of the Kpna7 protein in oocytes of different developmental stages was analyzed by immunohistochemistry. As shown in Fig. 3A, staining signal for Kpna7 
protein was detected in oocytes of different stages. The protein appears to be predominantly localized to the nucleus of small oocytes at pre- and early vitellogenic stages. No specific signal was detected in oocytes of different stages in the ovarian section incubated with antibody pre-absorbed with Kpna7 peptide antigen (Fig. 3B).

Identification of Kpna7 interacting proteins by yeast two-hybrid screening To further explore the functional network of Kpna7, we used yeast two-hybrid system to search for Kpna7 interacting proteins. Using Kpna7 as a bait, we screened a rainbow trout egg cDNA library. Two yeast clones were selected as a result of their survival on high stringency plates. Both clones showed LacZ activity in a $\beta$-galactosidase colony-lift filter assay (Fig. 4A). DNA sequencing analysis of the rescued plasmids revealed that one clone encodes rhamnose-binding lectin 3 (Stl3), and the other one contains a sequence encoding an uncharacterized protein (GenBank accession number: CR362149). To test the binding affinity of the identified proteins with Kpna7 protein, a $\beta$-galactosidase CPRG assay was performed. As shown in Fig. 4B and 4C, the $\beta$-galactosidase activities in yeast cells harboring either the stl3 plasmid or the unknown gene plasmid were significantly higher than those in the control cells, confirming the interactions of Kpna7 with these two proteins. RT-PCR analysis showed that stl3 expression is restricted to eggs, and the unknown gene is specifically expressed in eggs and testis (Fig. 4D). As revealed by real-time PCR, the expression patterns of stl3 and the unknown gene during embryonic development are similar, being extremely high in unfertilized eggs and decreasing drastically in embryos after fertilization (Fig. 4E and 4F). 
Kpna7 interacts with and facilitates nuclear import of Stl3

Using the online algorithm cNLS Mapper (http://nls-mapper.iab.keio.ac.jp/cgibin/NLS_Mapper_form.cgi) (Kosugi et al. 2009b), three overlapping NLSs were predicted at the C-terminus of St13 (Fig. 5A). To test the binding characteristics of the putative NLS cluster of Stl3 with Kpna7, Myc-tagged Stl3 (either wild-type or mutant) and Flag-tagged Kpna7 were co-expressed in HEK 293 cells followed by coimmunoprecipitation with anti-Flag antibody. As shown in Fig. 5B, the wild-type Stl3 is capable of interacting with Kpna7, while the mutant with the C-terminal NLS cluster deleted fails to bind to Kpna7. This result indicates that the interaction between Kpna7 and St13 is accomplished through the C-terminal NLS region of Stl3 protein.

To investigate whether Kpna7 could facilitate the nuclear transportation of Stl3 through interaction with the NLS cluster, HEK 293 cells were co-transfected with the Kpna7 expression construct (pcDNA3.1-Kpna7) along with the EGFP-tagged constructs expressing either the wild-type Stl3 (pEGFP-Stl3-wt) or the mutant St13 (pEGFP-St13$\Delta \mathrm{NLS})$. Nuclear localization of St13 was observed in cells overexpressing both wild-type Stl3 and Kpna7 (Fig. 6A-C). However, the mutant Stl3 showed a predominant cytoplasmic distribution in the cells overexpressing the mutant Stl3 and Kpna7 (Fig. 6DF). Localization of Stl3 in the cells without exogenous Kpna7 is mainly cytoplasmic (Fig. 6G-I). These data suggest that Kpna7 helps facilitate the nuclear transportation of Stl3, and the C-terminal NLS cluster is required for this process. 


\section{Discussion}

The finely orchestrated development of oocyte and early embryos has been the focus of many studies in mammals, in which a number of essential oocyte-specific genes have been identified (Sun et al. 2008; Zheng and Dean 2007). However, studies of egg-specific genes in fish are limited. Based on analysis of EST sequences from a rainbow trout egg cDNA library, we have previously identified and characterized three novel egg-specific genes in rainbow trout that include oorp-t (Ramachandra et al. 2007), rtgst-1 (Qiu et al. 2008) and fbxoo (Wang et al. 2013). In this study, we report the characterization of another egg-specific gene, kpna7, in rainbow trout. The predicted rainbow trout Kpna7 protein shares low sequence identity with its mammalian counterparts, but contains characteristic functional domains for importin $\alpha$ family members in mammals. Like human (Kelley et al. 2010) and bovine Kpna7 (Tejomurtula et al. 2009), rainbow trout Kpna7 also contains seven ARM repeats instead of eight ARM repeats present in other members of the importin $\alpha$ family.

Expression of kpna7 mRNA is predominantly in eggs as revealed by RT-PCR analysis (Fig. 1). This is in agreement with results from studies of bovine (Tejomurtula et al. 2009), mouse (Hu et al. 2010) and porcine KPNA7 genes (Wang et al. 2011). Minor expression of kpna7 mRNA in rainbow trout testis was also observed. Future analysis of its expression at different stages of testis development would provide valuable information on its specific role in transport of cargo proteins during spermatogenesis. Different KPNA genes showing distinct expression patterns during mouse spermatogenesis have been reported previously (Hogarth et al. 2006). 
Kpna7 is a divergent member of the Kpna family. It shares only $57 \%$ amino acid sequence identify with Kpna2, which is most closely related to Kpna7 (Supplementary Figure S1). This allowed us to generate a specific antibody against Kpna7 by immunizing rabbits with a synthetic peptide that is not only antigenic but also specific to Kpna7 relative to other Kpna proteins. Western blot analysis using the antibody specifically detected Kpna7 protein in the ovary but not in other somatic tissues (Fig. 2), indicating specificity of the antibody for its intended epitope. The specificity of the antibody was further demonstrated by showing no specific signal in oocytes of different stages in the ovarian section incubated with pre-absorbed antibody (Fig. 3B).

The regulated expression of importin a genes controls the transitions from one developmental stage to the next. Different expression patterns of importin $\alpha$ genes have been observed during embryonic development in pig (Wang et al. 2011) and differentiation of mouse embryonic stem cells (Yasuhara et al. 2013). In this study, we examined the expression profile of kpna7 mRNA during early embryonic development and observed high expression of kpna7 mRNA in unfertilized eggs and early stages of embryos but barely detectable expression in day 4 embryos and thereafter. This expression pattern is similar to bovine KPNA7 (Tejomurtula et al. 2009) and a number of known maternal-effect genes in mammals (Burns et al. 2003; Pennetier et al. 2004), suggesting that rainbow trout kpna7 is a maternal-effect gene required for activation of embryonic genome during early development.

The essential role of Kpna7 in early embryonic development has been well established (Hu et al. 2010; Tejomurtula et al. 2009; Wang et al. 2011). However, the cargo proteins that are transported into the nucleus by Kpna7 during the process are largely unknown. 
The egg-specific expression of Kpna7 and its requirement for early development led us to hypothesize that Kpna7 is responsible for transporting egg-specific nuclear factors important for early embryonic development. To test this hypothesis, we performed yeast two-hybrid screening to identify Kpna7-interacting proteins using a rainbow trout egg cDNA library. Of the two proteins identified, Stl3 is encoded by a known gene that has been reported in several fish species (Lam and Ng 2002; Shiina et al. 2002; Tateno et al. 2001). STL3 is one of the three L-rhamnose-binding lectins that are known to have a variety of biological functions in fish eggs including prevention of polyspermy, regulation of carbohydrate metabolism, and cross-linking of carbohydrate-rich proteins of the fertilization envelope (Tateno et al. 2002b). Both Stl3 mRNA and protein could be detected in cortical vesicles of previtellogenic oocytes (Tateno et al. 2002a; Tateno et al. 2002b). Northern blot analysis revealed that stl3 expression is restricted to eggs in steelhead trout (Tateno et al. 2002b). This was confirmed in the present study showing specific expression of stl3 mRNA in rainbow trout eggs (Fig. 4). The uncharacterized protein that interacts with Kpna7 appears to be gonad-specific and is highly abundant in unfertilized eggs (Fig. 4), suggesting an important role of this protein in the development of early embryos in rainbow trout.

The interactions between importin $\alpha$ and cargo proteins are accomplished by the network of amino acids that create the NLS and its binding surface. Although the NLS-binding domains of different importin $\alpha$ proteins are conserved, each importin $\alpha$ appears to have distinct NLS-binding specificities (Jans et al. 2000). The unique structure of Kpna7 (7 ARM repeats) may suggest a distinct role of this protein in transporting specific cargo proteins. In vitro binding assays showed that human and porcine KPNA7 do not bind to 
the NLS from the SV40-T antigen, a classical monopartite NLS for importin $\alpha$ proteins (Kelley et al. 2010; Wang et al. 2011). However, other evidence indicates that bovine KPNA7 is able to bind to nucleoplasmin 2 (NPM2), which contains a classical bipartite NLS (Tejomurtula et al. 2009). A recent study demonstrated that porcine KPNA7 interacts with sperm associated antigen 17-like protein (SP17) and ras responsive element binding protein 1, transcript variant 1 (RREB), both of which contain putative NLS (Park et al. 2012). The present study showed that interaction of rainbow trout Kpna7 with Stl3 is through a non-classical NLS located at the C-terminus of St13, indicating that Kpna7 may transport specific proteins with uncharacterized NLSs. Using an EGFP reporter assay, the present study also demonstrated that Kpna7 can facilitate the nuclear transportation of Stl3 in HEK 293 cells. However, we have to acknowledge that the intracellular proteins in human cells used in the experiment are not the same as those in rainbow trout eggs/embryos. A better approach to evaluate the role of Kpna7 in the transportation of oocyte-specific nuclear factors would be performing oocyte/egg microinjection assays.

In conclusion, we have characterized rainbow trout kpna7 cDNA and demonstrated that kpna7 is predominantly expressed in rainbow trout eggs and early embryos. We have also provided evidence indicating that Kpna7 interacts with egg-specific proteins and facilitates nuclear import of a known egg-specific factor, St13. Thus, we suggest that Kpna7 plays an important role in the nuclear transport of key egg-specific nuclear proteins during early development. 


\section{Materials and Methods}

Collection of fish samples

Mature eggs were collected by stripping from three female fish at spawning at the National Center for Cool and Cold Water Aquaculture (Kearneysville, WV). The eggs were artificially fertilized and incubated at $13{ }^{\circ} \mathrm{C}$ in a flow through system. Embryonic samples were collected at a series of time points after fertilization, including $0 \mathrm{~h}, 3 \mathrm{~h}, 7.5 \mathrm{~h}$, 11.5h, 18h, 27h, 34h, 2d, 3d, 4d, 5d, 6d, 8d, 10d, 12d, 16d and 25d post fertilization. Various tissue samples including heart, gill, testis, muscle, kidney, liver, stomach and spleen were collected from mature fish. Ovarian samples at pre-vitellogenesis $(\leq 0.65$ $\mathrm{mm})$, early-vitellogenesis $(0.65-1.1 \mathrm{~mm})$, mid-vitellogenesis $(1.1-2.1 \mathrm{~mm})$ and latevitellogenesis (2.1-4.0 $\mathrm{mm}$ ) were collected as described previously (Ramachandra et al. 2007). All samples were quick frozen in liquid nitrogen and stored in $-80{ }^{\circ} \mathrm{C}$ until use.

\section{Molecular cloning of Kpna7}

To identify the rainbow trout kpna7 cDNA sequence, a BLAST search of the Gene Indices database (Dana Farber Cancer Institute) was performed using bovine KPNA7 cDNA sequence as a query. A TC sequence containing the rainbow trout kpna7 cDNA was identified. Multiple EST sequences representing the TC sequence were identified from the database. An EST clone that contains the longest insert was retrieved from an arrayed rainbow trout egg cDNA library constructed previously (unpublished data). The insert of the clone was completely sequenced by primer walking.

Reverse transcription polymerase chain reaction (RT-PCR) 
Total RNA from various tissues was isolated using Trizol reagent (Invitrogen, Carlsbad, CA) according to the manufacturer's instructions. Isolated total RNA was treated with DNase I (Promega Madison, WI) followed by cDNA synthesis using Oligo (dT)18 primer and SuperScript III reverse transcriptase (Invitrogen, Carlsbad, CA). A negative control reverse transcription reaction (without reverse transcriptase) using pooled RNA was conducted to confirm no genomic DNA contamination in the RNA preparations. RTPCR was performed in a $25-\mu l$ reaction using gene specific primers (Supplementary Table S1) with the condition of a 5 min denaturation at $94{ }^{\circ} \mathrm{C}$ followed by 30 cycles of 94 ${ }^{\circ} \mathrm{C}$ for $30 \mathrm{sec}, 58^{\circ} \mathrm{C}$ for $45 \mathrm{sec}$, and $72{ }^{\circ} \mathrm{C}$ for $30 \mathrm{sec}$, and a final extension at $72{ }^{\circ} \mathrm{C}$ for 10 min. Rainbow trout $\beta$-actin (actb) gene was used as an internal control for RNA quantity.

Quantitative rea-time polymerase chain reaction

Total RNA from eggs/embryos was isolated using Trizol reagent (Invitrogen, Carlsbad, CA) followed by additional purification steps with lithium chloride precipitation. After DNase treatment, the RNA was used for cDNA synthesis using a mixture of oligo (dT)18 primer and random hexamer, and SuperScript III reverse transcriptase (Invitrogen, Carlsbad, CA). Gene specific primers used in the analysis are shown in Supplementary Table S1. Quantitative real-time PCR was performed on a Bio-Rad CFX96 ${ }^{\mathrm{TM}}$ Real-Time PCR Detection System using iQ ${ }^{\mathrm{TM}}$ SYBR ${ }^{\circledR}$ Green Supermix (Bio-Rad, Hercules, CA) in a $10-\mu 1$ reaction. Rainbow trout $18 \mathrm{~S}$ ribosomal RNA (18S rRNA) and hypoxanthineguanine phosophoribosyltransferase (hprt) genes were used as endogenous controls. Cycling parameters were $95^{\circ} \mathrm{C}$ for $3 \mathrm{~min}$. followed by 40 cycles of $95^{\circ} \mathrm{C}$ for $10 \mathrm{sec}$. and $60{ }^{\circ} \mathrm{C}$ for $1 \mathrm{~min}$. Melting curve analyses were programmed after amplification. Standard 
curves for each gene and the endogenous controls were constructed using serial dilutions of a pooled cDNA sample. The quantity of the target gene and the endogenous control genes in each sample was determined from respective standard curves. The quantity of the target gene mRNA was then normalized to the geometric mean of the reference genes. One-way analysis of variance (ANOVA) followed by the Tukey's multiple comparison tests were performed using the $\mathrm{R}$ software system to determine the significance of differences.

Generation of anti-Kpna7 antibody

The anti-Kpna7 antibody was prepared commercially by GenScript (Piscataway, NJ). A 15-amino acid synthetic peptide (EDAVQSPELNTDNHC) of the predicted amino acid sequence of rainbow trout Kpna7 was designed using OptimumAntigen ${ }^{\text {TM }}$ Design Tool (GenScript, Piscataway, NJ). The cysteine residue at the C-terminus was added to the peptide for conjugation to $\mathrm{KLH}$, and the conjugated peptide was used for immunization of two rabbits. The antibody was purified by affinity chromatography on columns containing peptide conjugated to affinity resin.

Western blot analysis

Frozen tissue samples were homogenized in T-PER protein extraction buffer containing Halt ${ }^{\mathrm{TM}}$ protease inhibitor cocktail (Thermo Fisher Scientific, Waltham, MA). Protein concentrations of the samples were determined using a Nanodrop1000 Spectrophotometer. Fifteen $\mu \mathrm{g}$ of protein from each sample were loaded on a Tris- $\mathrm{HCl}$ ready gel (Bio-Rad, Hercules, CA). Electrophoresis and protein transfer were performed 
as previously described (Wang et al. 2013). After blocking overnight at $4^{\circ} \mathrm{C}$, the membrane was incubated with 1:1000 diluted anti-Kpna7 antibody and 1:1000 diluted anti- $\beta$-Tubulin monoclonal antibody (Sigma-Aldrich, St. Louis, MO) for 2 h. Following three washes with TBST, the membrane was incubated with IRDye $800 \mathrm{CW}$ goat antirabbit IgG and IRDye 680LT goat anti-mouse IgG antibodies (Li-COR, Lincoln, NE) for $20 \mathrm{~min}$. Detection of the proteins was performed following standard instruction of the Odyssey system (Li-COR, Lincoln, NE).

Immunohistochemistry

Fixed ovarian samples were embedded in paraffin, sectioned, and mounted onto glass slides. The paraffin slides were de-paraffinized and rehydrated followed by treatment with $0.3 \% \mathrm{H} 2 \mathrm{O} 2$ in methanol to quench the endogenous peroxidase activity. The ABC Peroxidase Staining kit and Metal Enhanced DAB Substrate kit (Thermo Fisher Scientific) were used to detect and visualize the protein signals according to the manufacturer's instructions. The rabbit anti-Kpna7 polyclonal antibody was used as a primary antibody in the analysis. The negative control section was incubated with antiKpna7 antibody pre-absorbed with an excess of Kpna7 peptide (1:10 molar ratio).

\section{Yeast two-hybridization}

The Matchmaker Two-Hybrid System (Clontech Laboratories, Mountain View, CA) was used to identify proteins interacting with Kpna7 according to the manufacturer's instructions. To generate the bait expression vector, the coding region of kpna7 was cloned in frame in the pGBKT7 vector (Clontech Laboratories) at the NdeI and BamHI 
sites. Yeast AH109 competent cells were co-transformed with a SMART PCR amplified rainbow trout oocyte cDNA library, a linearized pGADT7 plasmid and the bait expression plasmid (pGBKT7-Kpna7). Yeast cells were plated on synthetic dropout selection medium lacking histidine, leucine and tryptophan (med dropout plate: $\mathrm{SD}$ /-his/leu/-try) and incubated at $30{ }^{\circ} \mathrm{C}$ for 3 days. Single colonies $(>2 \mathrm{~mm})$ were selected and streaked on fresh high dropout plates (high dropout plate: $\mathrm{SD} /$-ade/-his/-leu/-try). Plasmid was isolated from high dropout medium of each single colony and was transformed into E. coli competent cells using ampicillin as antibiotic to select for pGADT7 resistant clones. The ampicillin resistant plasmids were sequenced and the sequences were used to BLAST the GenBank database. To confirm the screening results, the rescued plasmids (expressing both $\mathrm{BD}$ and $\mathrm{AD}$ binding proteins) were retransformed into host strain and plated on high dropout plates. The transformants were tested for $\beta$-galactosidase activity by both the filter lift assay and the yeast $\beta$-galactosidase liquid assay using CPRG (cholorophenol red- $\beta$-d-galacto-pyranoside) according to the manufacturer's instructions. The $\beta$-galactosidase units were calculated using the following formula: $\beta$-galactosidase units $=1000 \mathrm{x}$ OD578/ ( $\mathrm{x}$ V x OD600) where OD578 is the absorbance of cholorophenol red and OD600 is the cell density at the start point; $t$ is elapsed time (in minute) of incubation; $\mathrm{V}$ is $0.1 \mathrm{x}$ concentration factor. 1 unit of $\beta$-galactosidase is defined as the amount that hydrolyzes $1 \mu \mathrm{mol}$ of CPRG to cholorophenol and d-galactose per minute per cell.

Construction of expression vectors 
The coding regions encoding the wild type rainbow trout kpna7 and stl3, as well as the mutant stl3 (encoding 1-372 aa) were PCR amplified using gene-specific primers containing BamHI/XhoI sites (Table1). The reverse primer for the kpna7 coding region contains a sequence encoding a Flag tag and a stop codon. The coding sequence for kpna7 was cloned into pcDNA3.1/Myc-His vector (Life Technologies, Carlsbad, CA) to generate the pcDNA3.1-Kpna7-Flag construct. The coding sequences (minus stop codon) for both the wild type and mutant stl3 were cloned in frame with the Myc tag sequence in pcDNA3.1/Myc-His vector to generate the pcDNA3.1-Stl3-wt-Myc and pcDNA3.1-St13$\Delta$ NLS-Myc constructs, respectively. The same sequences were also cloned in frame with the EGFP sequence in pcDNA3-EGFP vector (Addgene, Cambridge, MA) to generate the pcDNA3-EGFP-Stl3-wt and pcDNA3-EGFP-Stl3- $\triangle$ NLS constructs, respectively. All constructs were confirmed by sequencing.

\section{Co-immunoprecipitation}

HEK 293 cells were maintained in Dulbecco Modified Eagle Medium (DMEM) supplemented with $10 \%(\mathrm{v} / \mathrm{v})$ fetal bovine serum (Life Technologies, Carlsbad, CA). Cells were transiently transfected with the construct expressing Flag-tagged Kpna7 (pcDNA3.1-Kpna7-Flag) and the constructs expressing either the Myc-tagged wild type St13 (pcDNA3.1-St13-wt-Myc) or the Myc-tagged mutant Stl3 (pcDNA3.1-Stl3- $\Delta$ NLSMyc) using X-tremeGENE 9 transfection reagent (Roche, Mannheim, Germany) according to the manufacturer's protocol. Cells were collected $24 \mathrm{~h}$ after transfection. Whole cell lysates were immunoprecipitated according to the instructions of the Pierce 
co-immunoprecipitation kit (Life Technologies, Carlsbad, CA) with anti-Flag antibody and analyzed by Western blotting with anti-Myc antibody (Genscript, Piscataway, NJ).

\section{EGFP reporter assay}

HEK 293 cells were seeded on 20 mm diameter Poly-D-lysine coated German coverslips (Neuvitro, El Monte, CA) placed inside 6-well plates. Twenty four h after plating, cells were co-transfected with the Kpna7 expression construct (pcDNA3.1-Kpna7) along with the EGFP constructs expressing either the EGFP-tagged wild type Stl3 (pEGFP-Stl3-wt) or the mutant Stl3 (pEGFP-Stl3- $\Delta$ NLS). Cells were also transfected with the pEGFPStl3-wt construct alone. Forty eight $\mathrm{h}$ after transfection, cells on coverslips were washed with PBS and fixed in methanol for 5 min. Nuclear DNA of the cells was stained with DAPI and the cells were analyzed with a fluorescence microscope (Zeiss Axio imager M1).

\section{Acknowledgments}

This study was supported by the USDA ARS Cooperative Agreement No. 58-1930-0059. It is published with the approval of the director of the West Virginia Agricultural and Forestry Experiment Station as scientific paper No. 3220.

\section{Figure legends}

Figure 1. Analysis of kpna7 mRNA expression. A: Tissue distribution of kpna7 mRNA assessed by RT-PCR. Trout tissues tested include gill (Gi), kidney (Ki), liver (Li), muscle $(\mathrm{Mu})$, spleen (Sp), testis (Te) and eggs (Eg). M: 100 bp DNA marker; -RT: RT negative 
control. Trout actb was used as a control for RNA quality. B: RT-PCR analysis of kpna2, kpna2-like, kpna4 and kpna5 mRNA expression in rainbow trout tissues including include gill (Gi), kidney (Ki), liver (Li), muscle (Mu), spleen (Sp), testis (Te) and eggs (Eg). C: Quantitative real-time PCR analysis of kpna7 mRNA expression during ovarian development. Ovarian samples at different developmental stages (pre-, early-, mid- and late-vitellogenesis) were used in the analysis. The quantity of kpna7 mRNA was normalized to the quantity of actb gene. The means of the normalized gene expression values $(\mathrm{n}=4)$ for each stage of ovarian development were calculated and expressed as relative abundance. Different letters indicate significant difference $(\mathrm{P}<0.05)$. D: Quantitative real-time PCR analysis of kpna7 mRNA expression during embryonic development. Embryonic samples analyzed include unfertilized eggs and different stage embryos collected $0 \mathrm{~h}, 3.5 \mathrm{~h}, 7.5 \mathrm{~h}, 11.5 \mathrm{~h}, 13.5 \mathrm{~h}, 18 \mathrm{~h}, 27 \mathrm{~h}, 34 \mathrm{~h}, 2 \mathrm{~d}, 3 \mathrm{~d}, 4 \mathrm{~d}, 5 \mathrm{~d}, 6 \mathrm{~d}, 8 \mathrm{~d}, 10 \mathrm{~d}$, $12 \mathrm{~d}, 16 \mathrm{~d}$ and $25 \mathrm{~d}$ post fertilization. The quantity of kpna7 mRNA was normalized to the quantity of $18 \mathrm{~S}$ rRNA and hprt genes. The means of the normalized gene expression values $(n=4)$ for each stage of embryo were calculated and expressed as relative abundance. Different letters indicate significant difference $(\mathrm{P}<0.05)$.

Figure 2. Western blot analysis of Kpna7 protein expression in rainbow trout tissues. Tissue samples analyzed include gill (Gi), heart (He), kidney (Ki), liver (Li), spleen (Sp), stomach (St) and ovary (Ov). $\beta$-Tubulin (Tubb) serves as a control.

Figure 3. Immunohistochemical analysis of Kpna7 protein expression in oocytes of different developmental stages. A: Detection of Kpna7 protein expression signals in oocytes at different stages of development using anti-Kpna7 antibody. B: Ovarian section 
incubated with anti-Kpna7 antibody pre-absorbed with Kpna7 peptide antigen (negative control). C: Ovarian section stained with Hematoxylin and Eosin to show oocytes of different vitellogenic stages. The scale bars indicate $900 \mu \mathrm{m}$.

Figure 4. Identification of Kpna7 interacting proteins by yeast two-hybrid screening. A: Colony-lift filter assays showing $\beta$-galactosidase activities in yeast cultures expressing both Kpna7 protein and its interacting proteins (St13 or an uncharacterized protein). B-C: Yeast $\beta$-galactosidase liquid assays confirming interactions between Kpna7 and its binding proteins (mean \pm S.E. $n=3)$. Asterisks indicate significant difference $(\mathrm{P}<0.05)$. D: RT-PCR analysis of stl3 and unknown gene transcripts in rainbow trout tissues including gill (Gi), heart (He), kidney (Ki), liver (Li), muscle (Mu), spleen (Sp), testis (Te) and eggs (Eg). M: 100 bp DNA marker; -RT: RT negative control. Trout actb was used as a control for RNA quality. E-F: Quantitative real-time PCR analysis of stl3 and unknown gene transcript expression during embryonic development. The mRNA quantity of stl3 and unknown gene was normalized to the quantity of $18 \mathrm{~S}$ rRNA and hprt genes. The means of the normalized gene expression values $(n=4)$ for each stage of embryo were calculated and expressed as relative abundance. Different letters indicate significant difference $(\mathrm{P}<0.05)$.

Figure 5. Confirmation of interaction between Kpna7 and Stl3 by coimmunoprecipitation. A: Schematic representation of the Stl3 protein structure showing three overlapping NLSs at the C-terminal end of the protein. B: Co-immunoprecipitation of Myc-tagged Stl3 (wild type or mutant) with Flag-tagged Kpna7 from HEK293 cells 
co-transfected with pcDNA3.1-Kpna7-Flag and either pEGFP-St13-wt or pEGFP-St13$\Delta$ NLS. Immunoprecipitation was performed using anti-Flag antibody followed by Western blot analysis using anti-Myc antibody.

Figure 6. EGFP reporter assays showing Kpna7 facilitates the nuclear transportation of St13 through interaction with the NLS cluster. HEK 293 cells were co-transfected with the Kpna7 expression construct (pcDNA3.1-Kpna7) along with the EGFP constructs expressing either the EGFP-tagged wild type St13 (pEGFP-Stl3-wt) or the mutant Stl3 (pEGFP-Stl3-ANLS). Cells were also transfected with the pEGFP-Stl3-wt construct alone. The cells were grown on coverslips, and nuclear DNA was stained with DAPI and analyzed with a fluorescence microscope (Zeiss Axio imager M1). EGFP images (A, D, G), DAPI images (D, E, H), and the EGFP and DAPI-merged images (C, F, I) are shown. The scale bars indicate $40 \mu \mathrm{m}$.

\section{Supplementary Figure S1.}

Phylogenetic analysis of rainbow trout Kpna proteins. The molecular phylogenetic analysis was performed using MEGA 5.2 software (http://www.megasoftware.net/). Amino acid sequences for Kpna2 (TC15067), Kpna2-like (TC158343), Kpna3 (CX028092.1), Kpna4 (CA350405.1), Kpna5 (TC167152 and TC142045) and Kpna7 (KJ625239) were derived from cDNA sequences identified in GenBank (http://www.ncbi.nlm.nih.gov/genbank/) $\quad$ or $\quad$ Gene Index (http://compbio.dfci.harvard.edu/tgi/tgipage.html) database. 


\section{References}

Burns KH, Viveiros MM, Ren Y, Wang P, DeMayo FJ, Frail DE, Eppig JJ, Matzuk MM. 2003. Roles of NPM2 in chromatin and nucleolar organization in oocytes and embryos. Science 300:633-636.

Chook YM, Blobel G. 2001. Karyopherins and nuclear import. Curr Opin Struct Biol 11:703-715.

Dingwall C, Laskey RA. 1991. Nuclear targeting sequences--a consensus. Trends Biochem Sci 16:478-481.

Geles KG, Adam SA. 2001. Germline and developmental roles of the nuclear transport factor importin alpha3 in C. elegans. Development 128:1817-1830.

Hogarth CA, Calanni S, Jans DA, Loveland KL. 2006. Importin alpha mRNAs have distinct expression profiles during spermatogenesis. Dev Dyn 235:253-262.

Hood JK, Silver PA. 1998. Cse1p is required for export of Srp1p/importin-alpha from the nucleus in Saccharomyces cerevisiae. J Biol Chem 273:35142-35146.

Hu J, Wang F, Yuan Y, Zhu X, Wang Y, Zhang Y, Kou Z, Wang S, Gao S. 2010. Novel importin-alpha family member Kpna7 is required for normal fertility and fecundity in the mouse. J Biol Chem 285:33113-33122.

Itman C, Miyamoto Y, Young J, Jans D, Loveland K. 2009. Nucleocytoplasmic transport as a driver of mammalian gametogenesis. Semin Cell Dev Biol 20:607-619.

Jans DA, Xiao CY, Lam MH. 2000. Nuclear targeting signal recognition: a key control point in nuclear transport? Bioessays 22(6):532-544.

Kelley JB, Talley AM, Spencer A, Gioeli D, Paschal BM. 2010. Karyopherin alpha7 (KPNA7), a divergent member of the importin alpha family of nuclear import receptors. BMC Cell Biology 11:63.

Kosugi S, Hasebe M, Matsumura N, Takashima H, Miyamoto-Sato E, Tomita M, Yanagawa H. 2009a. Six classes of nuclear localization signals specific to different binding grooves of importin alpha. J Biol Chem 284:478-485.

Kosugi S, Hasebe M, Tomita M, Yanagawa H. 2009b. Systematic identification of cell cycle-dependent yeast nucleocytoplasmic shuttling proteins by prediction of composite motifs. Proc Natl Acad Sci USA 106:10171-10176.

Lam YW, Ng TB. 2002. Purification and characterization of a rhamnose-binding lectin with immunoenhancing activity from grass carp (Ctenopharyngodon idellus) ovaries. Protein Expr Purif 26:378-385.

Mason DA, Fleming RJ, Goldfarb DS. 2002. Drosophila melanogaster importin $\alpha 1$ and $\alpha 3$ can replace importin $\alpha 2$ during spermatogenesis but not oogenesis. Genetics 161:157170.

Otis KO, Thompson KR, Martin KC. 2006. Importin-mediated nuclear transport in neurons. Curr Opin Neurobiol 16:329-335.

Park KE, Inerowicz HD, Wang X, Li Y, Koser S, Cabot RA. 2012. Identification of karyopherin $\alpha 1$ and $\alpha 7$ interacting proteins in porcine tissue. PLoS One 7:e38990.

Pennetier S, Uzbekova S, Perreau C, Papillier P, Mermillod P, Dalbies-Tran R. 2004. Spatio-temporal expression of the germ cell marker genes MATER, ZAR1, GDF9, 
BMP15, andVASA in adult bovine tissues, oocytes, and preimplantation embryos. Biol Reprod 71:1359-1366.

Qiu GF, Weber GM, Rexroad CE, Yao J. 2008. Identification of RtGST-1, a novel germ cell-specific mRNA-like transcript predominantly expressed in early previtellogenic oocytes in rainbow trout (Oncorhynchus mykiss). Molecular reproduction and development 75:723-730.

Ramachandra RK, Lankford SE, Weber GM, Rexroad CE, Yao J. 2007. Identification of OORP-T, a novel oocyte-specific gene encoding a protein with a conserved oxysterol binding protein domain in rainbow trout. Mol Reprod Dev 74:502-511.

Schwoebel ED, Talcott B, Cushman I, Moore MS. 1998. Ran-dependent signal-mediated nuclear import does not require GTP hydrolysis by Ran. J Biol Chem 273:35170-35175.

Shiina N, Tateno H, Ogawa T, Muramoto K, Saneyoshi M, Kamiya H. 2002. Isolation and characterization of L-rhamnose-binding lectins from chum salmon (Oncorhynchus keta) eggs. Fisheries Science 68:1352-1366.

Sun Q, Liu K, Kikuchi K. 2008. Oocyte-Specific Knockout: A Novel In Vivo Approach for Studying Gene Functions During Folliculogenesis, Oocyte Maturation, Fertilization, and Embryogenesis. Biol Reprod 79:1014-1020.

Tateno H, Ogawa T, Muramoto K, Kamiya H, Hirai T, Saneyoshi M. 2001. A novel rhamnose-binding lectin family from eggs of steelhead trout (Oncorhynchus mykiss) with different structures and tissue distribution. Biosci Biotech Bioch 65:1328-1338.

Tateno H, Shibata Y, Nagahama Y, Hirai T, Saneyoshi M, Ogawa T, Muramoto K, Kamiya H. 2002a. Tissue-specific expression of rhamnose-binding lectins in the steelhead trout (Oncorhynchus mykiss). Biosci Biotech Bioch 66:1427-1430.

Tateno H, Yamaguchi T, Ogawa T, Muramoto K, Watanabe T, Kamiya H, Saneyoshi M. 2002b. Immunohistochemical localization of rhamnose-binding lectins in the steelhead trout (Oncorhynchus mykiss). Dev Comp Immunol 26:543-550.

Tejomurtula J, Lee K, Tripurani SK, Smith GW, Yao J. 2009. Role of Importin Alpha8, a New Member of the Importin Alpha Family of Nuclear Transport Proteins, in Early Embryonic Development in Cattle. Biol Reprod 81:333-342.

Thorgaard GH, Bailey GS, Williams D, Buhler DR, Kaattari SL, Ristow SS, Hansen JD, Winton JR, Bartholomew JL, Nagler JJ, Walsh PJ, Vijayan MM, Devlin RH, Hardy RW, Overturf KE, Young WP, Robison BD, Rexroad C, Palti Y. 2002. Status and opportunities for genomics research with rainbow trout. Comp Biochem Physiol B Biochem Mol Biol 133 :609-646.

Wang L, Tripurani SK, Wanna W, Rexroad CE, Yao J. 2013. Cloning and characterization of a novel oocyte-specific gene encoding an F-Box protein in rainbow trout (Oncorhynchus mykiss). Reprod Biol Endo 11:86.

Wang X, Park KE, Koser S, Liu S, Magnani L, Cabot RA. 2012. KPNA7, an oocyte-and embryo-specific karyopherin $\alpha$ subtype, is required for porcine embryo development. Reprod Fertil Dev 24:382-391

Weis K. 2003. Regulating access to the genome: nucleocytoplasmic transport throughout the cell cycle. Cell 112:441-451.

Yasuhara N, Shibazaki N, Tanaka S, Nagai M, Kamikawa Y, Oe S, Asally M, Kamachi Y, Kondoh H, Yoneda Y. 2006. Triggering neural differentiation of ES cells by subtype switching of importin- $\alpha$. Nat Cell Biol 9:72-79. 
Yasuhara N, Yamagishi R, Arai Y, Mehmood R, Kimoto C, Fujita T, Touma K, Kaneko A, Kamikawa Y, Moriyama T. 2013. Importin Alpha Subtypes Determine Differential Transcription Factor Localization in Embryonic Stem Cells Maintenance. Dev Cell 26:123-135.

Young JC, Major AT, Miyamoto Y, Loveland KL, Jans DA. 2011. Distinct effects of importin $\alpha 2$ and $\alpha 4$ on Oct3/4 localization and expression in mouse embryonic stem cells. FASEB J 25:3958-3965.

Zheng P, Dean J. 2007. Oocyte-specific genes affect folliculogenesis, fertilization, and early development. Semi Reprod Med 25:243-251. 
Table 1. Primers used in this study

\begin{tabular}{|c|c|c|}
\hline Primer name & Sequence & Application \\
\hline Kpna7-RT-F & GATGACCCCAGTCTGCAGTT & RT-PCR \\
\hline Kpna7-RT-R & ATAGGGAGGGAAGGGGTTCT & RT-PCR \\
\hline Kpna2-RT-F & GTGTGTTGAACTCTGGCGCGC & RT-PCR \\
\hline Kpna2-RT-R & GAGAAGTACTTCTCGATGAGGT & RT-PCR \\
\hline Kpna2like-RT-F & CTGCTTCGCCACCACAAGAGT & RT-PCR \\
\hline Kpna2like-RT-R & GCATATCCATCAGTTGTCAAG & RT-PCR \\
\hline Kpna4-RT-F & ACAGATGAACAGACCCAGGTG & RT-PCR \\
\hline Kpna4-RT-R & CATCCTTCACTGTCAGCAGGT & RT-PCR \\
\hline Kpna5-RT-F & GCACTACTTTGGTGTGGAGGA & RT-PCR \\
\hline Kpna5-RT-R & GCTGCAGGAAGTCTCAAACAC & RT-PCR \\
\hline Unknown-RT-F & TGTCCACCCAAGGTCAGCCC & RT-PCR \\
\hline Unknown-RT-R & ACTTTGGGCAGGTCTGTAGC & RT-PCR \\
\hline$\beta$-Actin-RT-F & AAGTGTGACGTGGACATCCGT & RT-PCR \\
\hline$\beta$-Actin-RT-R & TAATCCGCTGCTTCACCGTTC & RT-PCR \\
\hline Kpna7-realtime-F & AGAATCACCCCAGACACACC & Real time PCR \\
\hline Kpna7-realtime-R & ACTCAGGTGGAGGAGCTGAA & Real time PCR \\
\hline Stl3-realtime-F & AGCGTGCGAACTACGGTCGT & Real time PCR \\
\hline Stl3-realtime-R & CACTCGCTCTTCCCACCGCA & Real time PCR \\
\hline Unknown-realtime-F & CATGCAAGGTGGAGCTGAGA & Real time PCR \\
\hline Unknown-realtime- $\mathrm{R}$ & ACTTTGGGCAGGTCTGTAGC & Real time PCR \\
\hline 18S rRNA-F & TGCGGCTTAATTTGACTCAACA & Real time PCR \\
\hline $18 \mathrm{~S}$ rRNA-R & CAACTAAGAACGGCCATGCA & Real time PCR \\
\hline HPRT-realtime-F & GACCTCTTCTGCATCCCAAA & Real time PCR \\
\hline HPRT-realtime-R & GCCTCTCTGTCCTGTCAAGG & Real time PCR \\
\hline$\beta$-Actin-realtime-F & GCCGGCCGCGACCTCACAGACTAC & Real time PCR \\
\hline$\beta$-Actin-realtime- $R$ & CGGCCGTGGTGGTGAAGCTGTAAC & Real time PCR \\
\hline Kpna7-Y2-F & CATATGCCCACCACGAGTGCTACTG & Yeast two hybrid \\
\hline Kpna7-Y2-R & GGATCCTTAAAACCGGAAGGTCTTCT & Yeast two hybrid \\
\hline Kpna7-pcDNA-F & GGATCCGCCGCCACCATGGCCACCACGAGTGCTACTGA & Co-IP/EGFP \\
\hline Kpna7-pcDNA-Flag-R & CTCGAGTTACTTGTCATCGTCGTCCTTGTAGTCAAACCGGAAGGTCTTCTGCA & Co-IP/EGFP \\
\hline Stl3-pcDNA-F & GGATCCGCCGCCACCATGGGCATTTTGAGACTGACGGT & Co-IP/EGFP \\
\hline Stl3-pcDNA-R & CTCGAGGTCACAGGTGTAAGCCACAT & Co-IP/EGFP \\
\hline Stl3-pcDNA-R- $\Delta$ NLS & CTCGAGGATGCTGCTTATTGTTTCATGCT & Co-IP/EGFP \\
\hline
\end{tabular}




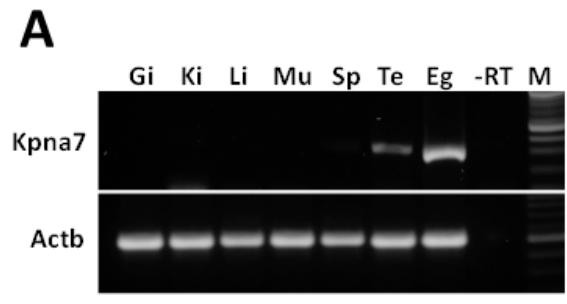

B

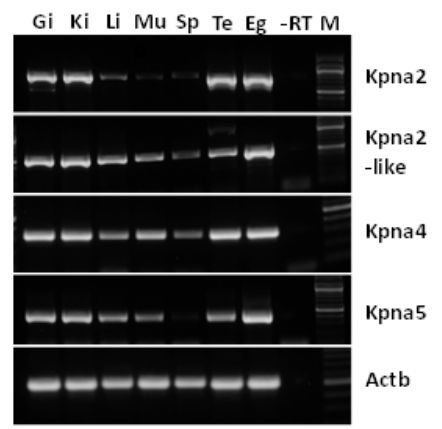

\section{C}

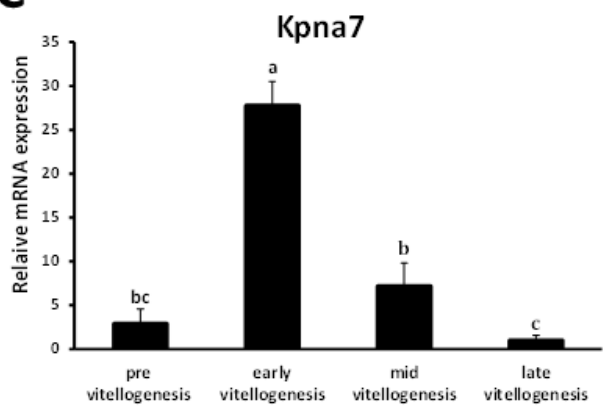

D

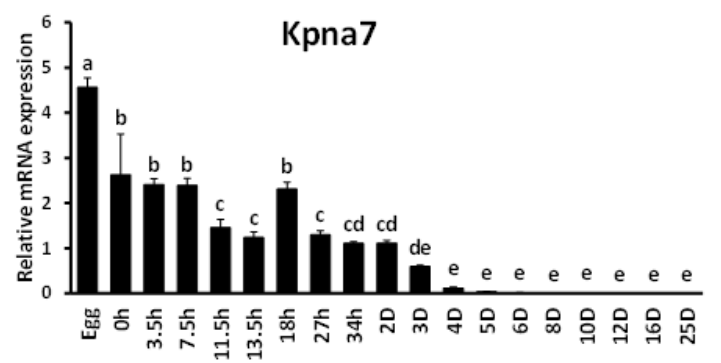

Figure 1 Oocyte-specific expression of KPNA 7 and its family members 


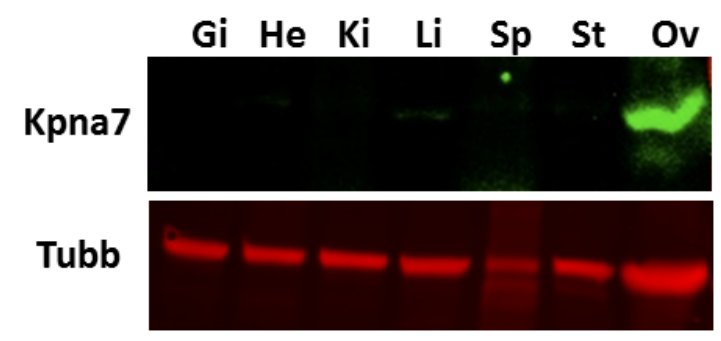

Figure 2. KPNA7 is a distinctive member of KPNA family and its subcellular localization within rainbow trout ovary 

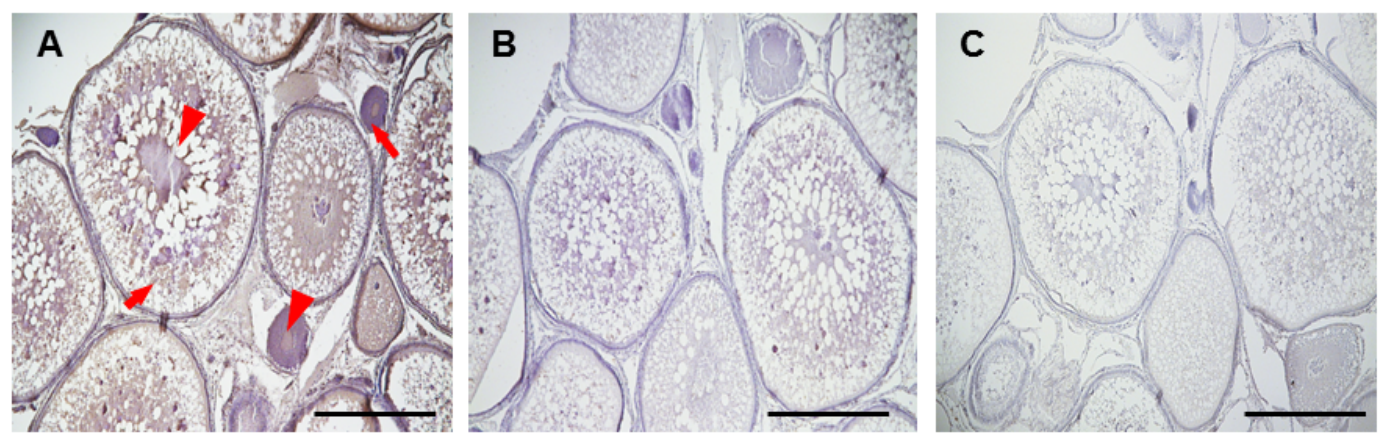

Figure 3. Expression profile of all the KPNA family member during embryogenesis of rainbow tout 
A

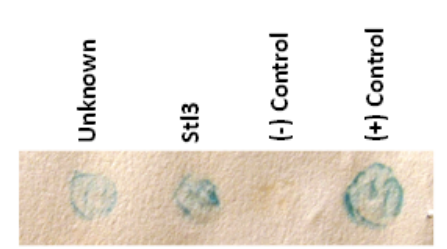

B

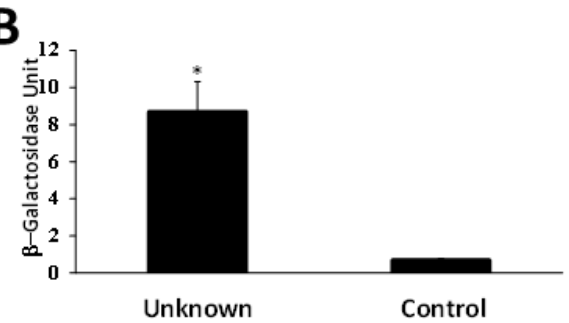

C

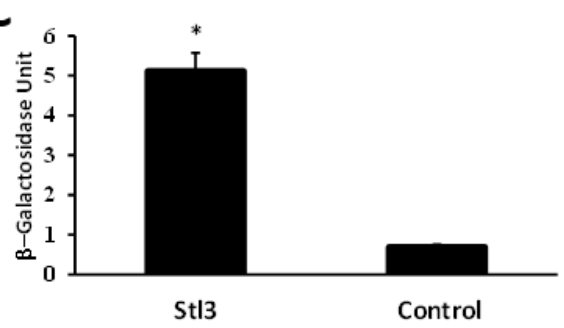

D

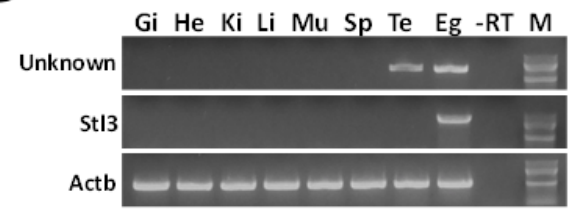

E

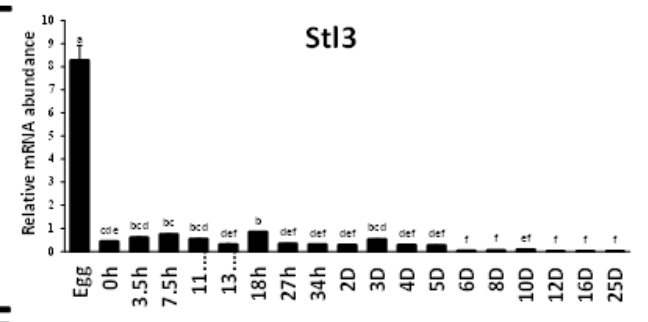

$\mathbf{F}$

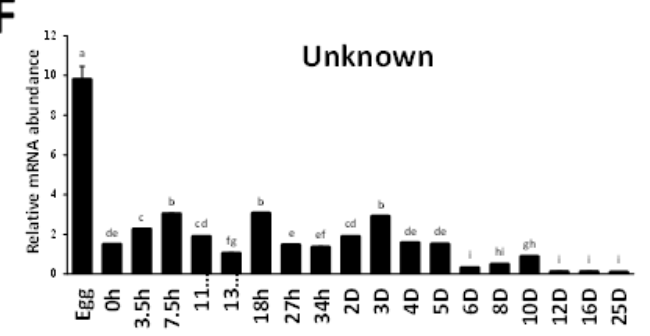

Figure 4. KPNA7 is interacting with two oocyte-specific genes Stl3 and the novel gene CR362149 
A

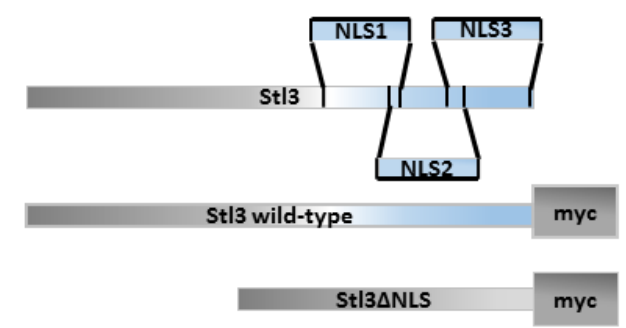

B

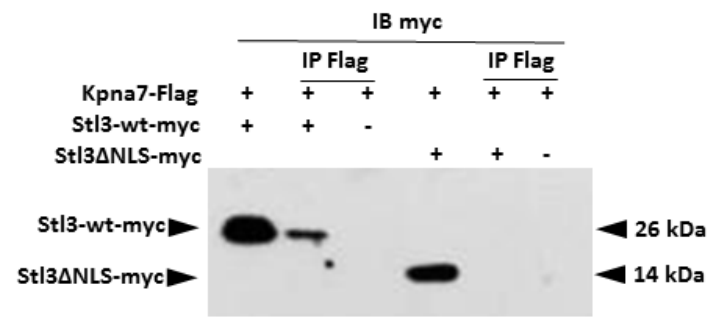

Figure 5. Stl3 forms a dimer structure harboring a NLS domain at C terminal that facilitate the interaction with KPNA7 


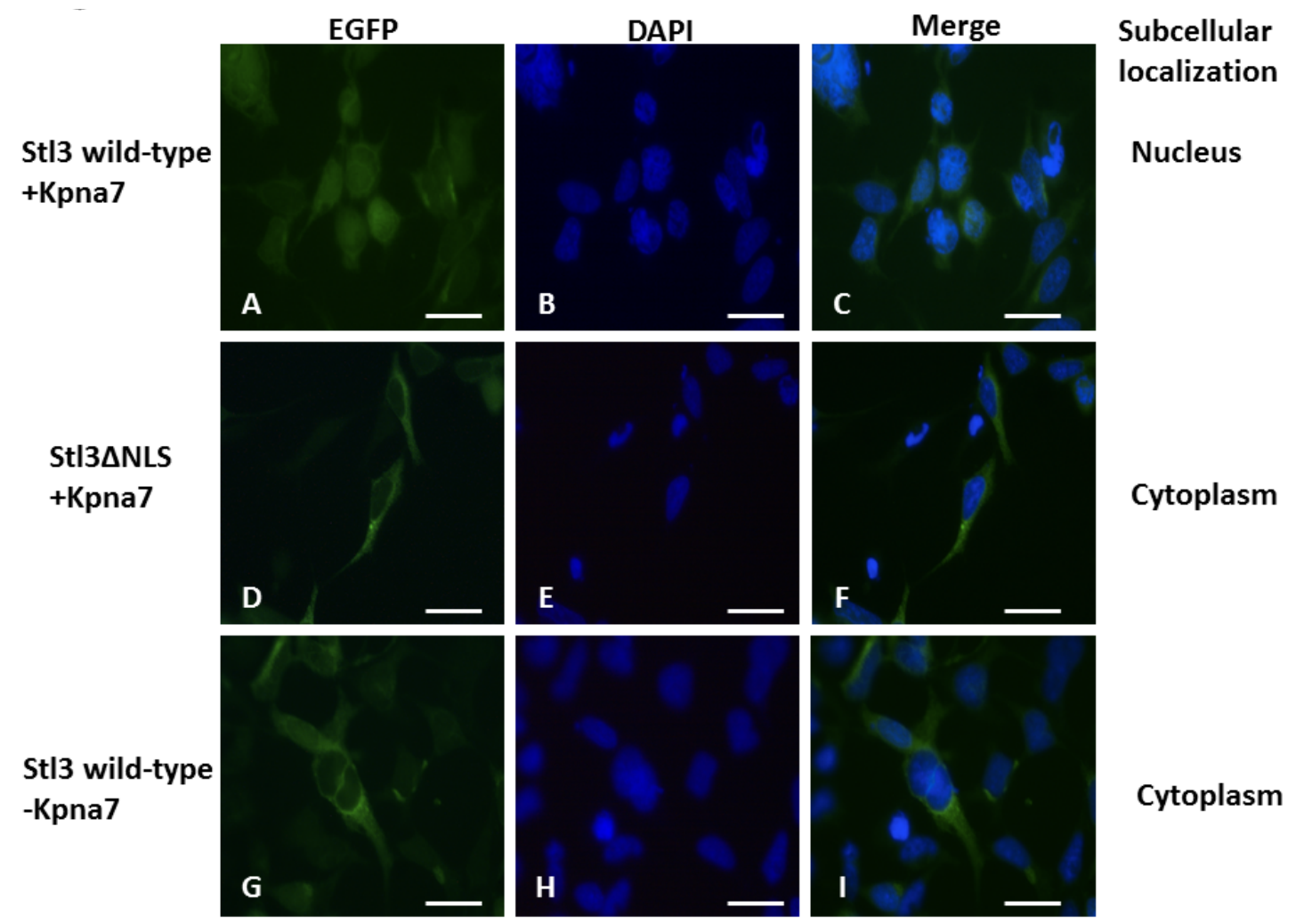

Figure 6. Stl3 and KPNA7 interact through a functional NLS 


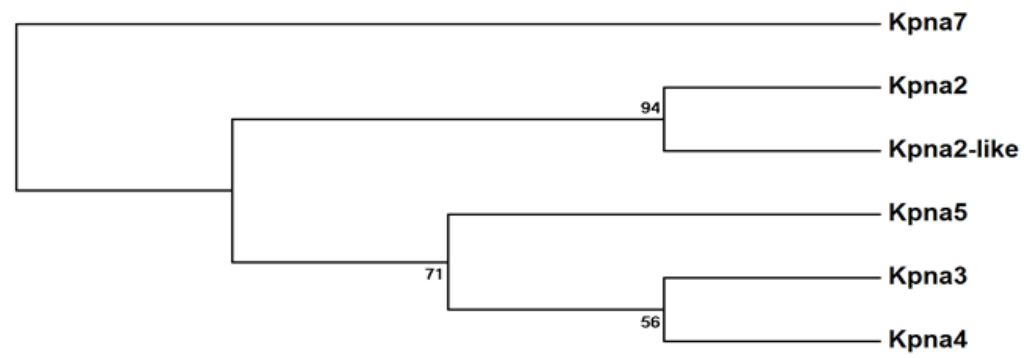

Figure S1 Full-length cDNA sequence of rainbow trout KPNA7 


\section{Chapter 2:}

DNA methylation and miRNA 1296 act in concert to mediate spatiotemporal expression of oocyte-specific KPNA7 during bovine oocyte and early embryonic development.

Lei Wang ${ }^{1}$, Jackie M. Hand ${ }^{1}$, Liyuan Fu ${ }^{1}$, George W. Smith ${ }^{2}$, Jianbo Yao ${ }^{1}$

${ }^{1}$ Laboratory of Animal Biotechnology and Genomics, Division of Animal and Nutritional Sciences, West Virginia University, Morgantown, WV 26506.

${ }^{2}$ Laboratory of Mammalian Reproductive Biology and Genomics, Departments of Animal Science and Physiology, Michigan State University, East Lansing, Michigan 48824.

Key Words: DNA methylation, miRNA, oocyte, early embryo development, maternal, cattle

* Corresponding author:

Jianbo Yao, Ph.D.

Tel: (304) 293-1948

e-mail: jianbo.yao@mail.wvu.edu

Plan to submit to Molecular Reproduction and Development 


\section{Abstract}

Epigenetic regulation of oocyte-specific maternal factors is essential for oocyte and early embryonic development. KPNA7 is one of the oocyte-specific maternal effect factors controlling nuclear uptake of essential nuclear factors. To elucidate whether single or multiple epigenetic mechanisms are involved in the controlled expression of KPNA7, both miRNA mediated mRNA degradation and DNA methylation associated transcriptional silencing of KPNA7 tissue- and developmental stage-specific expression were examined. During maternal to zygotic transition (MZT), previous studies in cattle revealed a miRNA-mediated mechanism to regulate expression maternal effect genes (such as Nobox, NPM2, Figla). Real-time PCR indicated an expression profile of KPNA7 mRNA similar to other maternal effect genes, which were dropped after embryonic genome activation (8-16 cells stage embryo). miRNA1296 was seen in all embryo samples and reached a peak level at the 8-16 cell embryo stage, coincident with the time of embryonic genome activation in cattle, when KPNA7 decreased. Ectopic coexpression of miRNA1296 and KPNA7 led to reduced KPNA7 protein expression and mutation on binding site around 1156-1176 bp interrupted the miRNA:mRNA interaction. These findings indicate miRNA1296 targeting coding region of KPNA7 is a potential regulator involved in KPNA7 maternal transcript degradation during MZT. Furthermore, DNA methylation profiles were investigated at the proximal promoter of KPNA7 in different somatic tissues and oocytes. Sequence analysis revealed one $\mathrm{CpG}$ island in the KPNA7 promoter and three oocyte-specific differential methylated sites. Expression of KPNA7 mRNA was reintroduced in bovine kidney-derived CCL2 cells, after treatment 
with the methylation inhibitor, 5-aza-2-deoxycytidine (5-Aza). Promoter region of CCL2 cells treated with 5-Aza was sequenced and revealed a lighter methylation rate in all the CpG sites compared to the non-treatment group. These results indicated that DNA methylation might account for oocyte-specific expression of KPNA7, whereas the decreased KPNA7 mRNA after MZT might be regulated by miRNA-mediated maternal transcripts, independent of DNA methylation. 


\section{Introduction}

Successful germ cell development and differentiation during oogenesis and early embryogenesis is accomplished through the help of nuclear proteins such as transcription factors and chromatin-remodeling factors that act in the nucleus (Itman, et al. 2009). A family of nuclear transporters called karyopherin (KPN) is the major player in the translocation of nuclear proteins through active, energy-dependent nuclear import system. To date, seven KPNAs have been identified in mammals; only Kpna7 was expressed strictly in oocytes and early embryos (Hu, et al. 2010a, Tejomurtula, et al. 2009, Wang, et al. 2011). In mice, KPNA7 knockout led to fetal lethality and sex imbalance. Abnormalities of epigenetic modifications (down-regulation of histone H3K27me3) are part of the Kpna7 knock out effects (Hu, et al. 2010a). In cattle and pigs, knockdown of KPNA7 significantly reduced blastocyst rate by inducing arrested embryonic development (Tejomurtula, et al. 2009, Wang, et al. 2011). KPNA7 showed a unique expression pattern, which was highly expressed in germinal vesicle (GV) oocytes through 8-cell stage embryos and dropped to barely detectable values in morula and blastocyst stage embryos. The sudden drop of mRNA during 8-16 cell stage is coincident with the time of maternal to zygotic transmission (MZT). All together, the function and unique expression pattern of KPNA7 revealed that it is a maternal factor critical for development in mammals. However, little is known about the mechanisms that control tissue- and stage-specific expression of KPNA7.

Maternal effect genes such as KPNA7, are the major driving force to facilitate oocyte maturation, fertilization and embryonic genome activation (Li, et al. 2010). However, 
after MZT, almost $90 \%$ of the maternal transcripts are degraded and the clearance of maternal transcripts is essential for normal embryonic development (Paynton, et al. 1988). For example, in Xenopus the oocyte-specific maternal transcript c-mos reduced quickly after fertilization and reintroducing c-mos protein to 2-cell stage embryos blocked development (Sagata, et al. 1989). This phenomenon was observed in mouse and other species, which indicated that maternal transcript degradation is obligatory for normal embryonic development (Alizadeh, et al. 2005). Multiple negative regulatory mechanisms including mRNA deadenylation, interaction with RNA-binding proteins and miRNA-mediated degradation are involved in post-transcriptional degradation of maternal transcripts. Such miRNAs as miRNA430 in zebrafish, miRNA427 in Xenopus and miRNA21 in rainbow trout are present prior to embryonic genome activation and further studies revealed more evidence that such miRNAs are responsible for degradation of hundreds of maternal transcripts (Bazzini, et al. 2012, Giraldez, et al. 2006, Lund, et al. 2009, Ramachandra, et al. 2008). In cattle, a growing body of evidence showed that oocyte-specific maternal mRNAs (NPM2, NOBOX and Figla) were targeted by miRNA (miRNA181a, miRNA196a, miRNA212) and led their degradation (Lingenfelter, et al. 2011, Tripurani, et al. 2011, Tripurani, et al. 2013). As a maternal factor, KPNA7 mRNA showed the typical drop at the MZT, therefore, the first goal of this study is to investigate if miRNA was involved in KPNA7 mRNA degradation during MZT.

Recent research revealed that DNA methylation at the 5-position of cytosine $(5 \mathrm{mC})$ largely occurs at $\mathrm{CpG}$ dinucleotides and is required for normal gamatogenesis and embryogenesis in mammals (Messerschmidt 2012). In early oogenesis, the genome of 
embryonic germ cells is dynamically reprogrammed during cell differentiation and the differential methylated regions (DMR) start to maintain the monoallelic expression of imprinted genes (Kass, et al. 1997, Maeda, et al. 2008, Panning and Jaenisch 1996). Genes of developmental importance, such as germ cell-specific factors Nanog, Dazl, Pou5f1 and Sry which control promordial germ cell development are all regulated through DNA methylation-mediated mechanisms (Hattori, et al. 2004, Maatouk, et al. 2006, Nishino, et al. 2004). Tissue-dependent and differentially methylated regions (TDMRs) are common in the mammalian genomes and corresponds to different cell types in the organism (Shiota 2004). The DNA methylation profile is tissue-specific, so it is reasonable to believe that methylation, in particular, of $\mathrm{CpG}$ sites located in the proximal promoter surrounding the transcription starting site (TSS), must play a role in controlling the expression of oocyte-specific maternal factors. As such, to investigate the DNA methylation status of KPNA7 between different tissue and oocyte might help to understand the controlling mechanism of tissue-specificity of KPNA7.

\section{Material and Method}

\section{Bioinformatics Analysis}

Microinspector http://bioinfo.uni-plovdiv.bg/microin- spector/, an algorithm for detection of possible interactions between miRNAs and targeted mRNA sequences, was used to predict putative binding sites between miRNA and their target. Full-length cDNA sequence of KPNA7 was used to upload to the software.

\section{Tissue collection, RNA isolation and microRNA expression analysis}


Bovine tissue sample collection, total RNA isolation and miRNA expression analysis in multiple tissues, oocytes and early embryos were performed as described previously (Tripurani, et al. 2010b)

\section{Plasmid construction}

Full-length bovine KPNA7 mRNA was amplified and cloned as described in (Tejomurtula, et al. 2009). PCR using gene-specific primers containing restriction sites of endonucleases BamHI/XhoI was performed and PCR products were cloned into pcDNA3.1 (Invitrogen, Carlsbad, CA) vector treated with the same two enzymes. Genomic sequence of pre-miR-1296 from the bovine genome was amplified using primers amplify $291 \mathrm{bp}$ of this area and containing BamHI/XhoI digestion sites. Products of PCR were digested and subcloned into pcDNA3.2 treated with same enzyme set. For construction of a vector containing KPNA7-ORF fused to the 3'end of a luciferase reporter, we used the dual luciferase pmirGLO vector (Promega, Madison, WI). KPNA7ORF was amplified from pcDNA3.1-KPNA7 with primer containing PmeI / XhoI sites and was subcloned into pmirGLO vector. Mutation of the mir-196a miRNA recognition element (MRE) in the NOBOX 3' UTR was performed using the QuickChange sitedirected mutagenesis kit (Stratagene, Santaclara, CA) according to the manufacturer's instructions.

\section{Cell culture and Reporter assay}

HEK293 cells were cultured in DMEM (Invitrogen, Carls- bad, CA) containing 10\% FBS. For transient transfection, Xtremegene 9 (Roche Applied Science, Indianapolis, IN) was used according to manufacturer's instructions. Following transfection, cells were 
incubated for $24 \mathrm{~h}$ before harvest for western blotting and luciferase assays. CCL22 cells were cultured in DMEM (Invitrogen, Carlsbad, CA) containing 10\% horse serum. 5-aza2-deoxycytidine (5-Aza) was added to the culture medium to a final concentration as 1

$\mu \mathrm{M}$ in a 6-well dish (Corning) seeded with cells, $24 \mathrm{~h}$ before the treatment. Treatment was continued for 3 days and fresh medium with 5-Aza was changed daily. After 3 days, cells were harvested and stored at $-80{ }^{\circ} \mathrm{C}$ until use.

\section{Western blot analysis luciferase assay}

Electrophoresis and transfer were performed according to a previous study with minor modifications (Wang, et al. 2013a). HEK293 cells were harvested with Pierce IP Lysis Buffer (Thermo, Rockford, IL), and $10 \mu \mathrm{l}$ was mixed with an equal volume of Laemmli sample buffer (Bio-Rad, Hercules, CA). Protein samples (15 $\mu \mathrm{g} / \mathrm{each})$ were separated on a 4-20\% gradient ready gel (Bio-Rad, Hercules, CA) and transfered onto an ImmobilonFL PVDF membrane (Millipore, Billerica, MA). Following transfer and blocking in 5\% nonfat dry milk in PBS containing $0.1 \%$ Tween-20 (PBST) for one hour, the membrane was then incubated in myc antibody (Genscript, Piscataway, NJ) and $\beta$-actin antibody (Genscript, Piscataway, NJ) both diluted into $0.5 \mu \mathrm{g} / \mathrm{ml}$ in blocking buffer shaking overnight at $4^{\circ} \mathrm{C}$. After three washes of primary antibody, two secondary antibodies IRDye $800 \mathrm{CW}$ goat anti-rabbit and IRDye680LT goat anti-mouse ((Li-COR, Lincoln, $\mathrm{NE}$ ) were added to blocking buffer and incubated for 20 minutes. Detection of the proteins was performed following standard instruction of the Odyssey system (Li-COR, Lincoln, NE). The luciferase assay was performed using the Dual-Glo luciferase assay system (Promega) following the instructions as described by the manufacturer. Firefly 
luciferase activity was normalized to renilla luciferase activity to adjust for variations in transfection efficiency among experiments. All transfection experiments were performed in quadruplicate $(n=4)$ with data averaged from four independent experiments.

\section{Bisulfite Sequencing}

Ten oocytes from primordial follicles and 5 pg DNA from somatic tissues (heart, lung, muscle, brain, intestines and spleen) were used. The DNA samples went through bisulfite treatment following the manufacturer's instruction of EZ DNA Methylation-Direct ${ }^{\mathrm{TM}}$ (Zymo, Research). Primers designed using the Methprimer online program were used in a $25 \mu 1 \mathrm{PCR}$ reaction for the first round of 40 cycles. Cycling conditions were $95^{\circ} \mathrm{C}$ for 9 min followed by 40 cycles of $95^{\circ} \mathrm{C}$ for $30 \mathrm{~s}$, annealing temperature $54^{\circ} \mathrm{C}$ for $30 \mathrm{~s}$, extension $72^{\circ} \mathrm{C}$ for $30 \mathrm{~s}$ and a final extension of $5 \mathrm{~min}$ at $72^{\circ} \mathrm{C}$. Nested PCR of 35 cycles were performed using the same PCR product as template in a higher annealing temperature (at $\left.58^{\circ} \mathrm{C}\right)$ and shorter denaturing time $\left(5 \mathrm{~min}\right.$ at $\left.95^{\circ} \mathrm{C}\right)$. The DNA regions of CpG sites were amplified and given the sampling bias of PCR, three independent PCR reactions were performed, purified, pooled together and cloned into pGemTeasy vector (Promega, WI). After verification by colony PCR, ten to twenty colonies for each DNA sample were sequenced. The sequencing results were analyzed using the online tool QUMA. Methylation sequencing PCR was performed using the same DNA samples from different somatic tissues.

\section{Results and Discussion}

\section{Identification of the KPNA7 promoter CpG island}


According to a previous study, KPNA7 contains 11 exons and 10 introns, located in bovine chromosome 25 and the $5^{\prime}$ upstream region of the KPNA7 gene conteins five putative E-box cis-regulatory elements (Tejomurtula, et al. 2009). Analysis of $1 \mathrm{~Kb}$ genomic DNA sequence up stream of the KPNA7 gene transcription starting site (TSS) using the online algorithm Methprimer revealed a putative $\mathrm{CpG}$ island containing $8 \mathrm{CpG}$ sites located (-221 to -114$)$ in the proximal promoter region of KPNA7, utilizing the general criteria for specific detection of $\mathrm{CpG}$ islands associated with the 5' end of genes. The parameters were minimum island length of $100 \mathrm{bp}, \mathrm{GC}$ content of minimum $50 \%$ and observed to expected $\mathrm{CpG}$ dinucleotide ratio of $50 \%$. In addition, all $\mathrm{CpG}$ sites within $1 \mathrm{~Kb}$ of KPNA7 TSS were identified (Figure 1.). It has been generally believed that DNA hypermethylation at the proximal promoter can repress gene transcription by interfering with transcription initiation (Borgel, et al. 2010). Therefore, this study was focused on 11 consecutive CpG sites, among them 8 sites were within the $\mathrm{CpG}$ island and 3 sites were located right before TSS at the CpG island shore region to screen out differential methylated sites which might contribute to the oocyte-specific expression of KPNA7.

Gene expression is controlled by the methylated $\mathrm{CpG}$ island on promoter region. Methylated CpG sites recruit other factors such as methyl-DNA binding proteins, which lead to condensation of chromatin resulting in transcription inactivation stage (Jones 2012). Recent studies discovered that, apart from $\mathrm{CpG}$ island, " $\mathrm{CpG}$ island shore" regions adjacent to $\mathrm{CpG}$ island in the promoter play a role in controlling gene expression. Unmethylated $\mathrm{CpG}$ island shore resulted in expression of genes such as Cav-1 in multiple cancer cell lines (Irizarry, et al. 2009, Rao, et al. 2013). In this study, two CpG sites were 
discovered in the $\mathrm{CpG}$ island shore, which is tightly linked to expression of KPNA7 in the oocyte, and this is the first report on $\mathrm{CpG}$ island shore regulating oocyte-specific gene.

\section{Differential methylation pattern of KPNA7 promoter in bovine oocyte and somatic}

\section{tissues.}

Using bisulfite conversion and subsequent DNA sequencing, the methylation status of 11 CpG sites were characterized (Figure 2). Ten clones were sequenced from each sample. Three CpG sites with high demethylation (60-70\%) were identified by comparing GV oocyte and 6 different somatic tissues (heart, lung, muscle, brain, intestines and spleen). These three oocye-specific hypomethylation sites $(-149,-63$ and -54$)$ were present only in the GV oocytes while most of them showed demethylation more than $20 \%$ in somatic tissues, which indicate a tissue-specific methylation pattern at these three sites.

Tissue-specific differential methylated regions (TDMR) have become an important aspect in epigenetic related gene expression studies (Canastar, et al. 2012, Song, et al. 2009). Comparisons between oocytes and sperm, developmental germ cell stages, and different embryonic stages showed stage or germ cell specific differential methylation region (Kobayashi, et al. 2012, Smallwood, et al. 2011). Moreover, methylation status of several factors important for oocyte development have been identified, but very few studies investigated the oocyte-specific methylation profile compared to other somatic tissues. In this study, it was demonstrated that methylation of $3 \mathrm{CpG}$ sites (-149, -63 and 54 ) in the promoter region is highly related to tissue specific expression of KPNA7. 


\section{Treatments with 5-Aza reintroduce expression of KPNA7 in CCL-22 cells}

5-Aza inhibits activity of DNA methyltransferase 1 (DNMT1) to maintain the status of DNA methylation. As cell divide, treatment with 5-Aza results in global demethylation in CCL-22 cell genome, resulting in the expression of genes silenced by DNA methylation (Jones 1985). CCL-22 cells were derived from bovine kidney cells, in which KPNA7 cannot be detected naturally. 5-Aza was used to treat CCL-22 cells, and expression of KPNA7 was induced, as shown in RT-PCR using primers targeting the KPNA7 $1.5 \mathrm{k}$ ORF (Figure 3a). The strongest KPNA7 expression was induced in 0.5 and $1 \mu \mathrm{M}$ groups and in 5 and $2.5 \mu \mathrm{M}$ weak expression was observed. The overall effect of 5-Aza treatments in CCL-22 cells showed a trend for a dose-dependence response with optimal concentration of $0.5 \mu \mathrm{M}$. With methytransferase inhibitor 5-Aza in the CCL-22 cell culture, genome methylation is reduced, which leads to reactivation of genes silenced by DNA methylation. This approach was developed decades ago and is widely adapted into different mammalian cells (Jüttermann, et al. 1994). In this study, after observed the presence of KPNA7 in CCL-22 cells, the DNA methylation profile of the proximal promoter was tested in the treatment group with the highest expression.

Bisulfite sequencing of CCL-22 cells from the $0.5 \mu \mathrm{M}$ and control $(0 \mu \mathrm{M})$ groups showed demethylation of the KPNA7 proximal promoter in the $0.5 \mu \mathrm{M}$ group, whereas in the control group hypermethylation was observed (Figure 3b), in agreement with the RTPCR results showing no band in the control group. Moreover, three oocyte-specific hypomethylated sites were all methylated in the the control group and in $0.5 \mu \mathrm{M}$ group they underwent partial demethylation mirroring to the methylation state in oocytes. These results in CCL-22 cells indicate that DNA methylation in the proximal promoter of 
KPNA7 is critical for tissue-specific expression of this gene. Moreover, it seems likely that the three $\mathrm{CpG}$ sites are highly correlated to KPNA7 tissue-specificity.

Specific CpG sites control of gene expression was observed in in prostate cancer cells. When treated with minimal concentration of 5-aza, one $\mathrm{CpG}$ site was hypersensitive to the challenge and methylation of this single site led to PMP24 gene silencing (Zhang, et al. 2010). There was a trend for demethylation of CpG sites to spread from TSS all the way to upstream direction. Two sites (-63 and -54$)$ closest to the TSS had greater demethylation than sites located upstream. Therefore, these two sites might play primary roles in relation to KPNA7 gene expression.

\section{Promoter demethylation does not activate KPNA7 expression after embryonic genome activation}

As a maternal effect factor, KPNA7 was expressed only in oocytes and the expression was high until it dropped rapidly after the MZT (from 16 cell stage) without coming back untill the formation of primordial germ cells in later pregnancy stage (Figure 4). Therefore, 16-cell and blastocyst genomic DNA samples were used to test if the silencing of KPNA7 is due to DNA methylation in the proximal promoter region. Bisulfite sequencing and subsequent analysis were performed according to the tissue-specificity studies. Results showed that at the 16-cell stage (the end of MZT) in cattle, the KPNA7 proximal promoter was heavily hypomethylated. At further developmental stages when the embryonic genome is completely activated, the KPNA7 proximal promoter was still extremely hypomethylated. This pattern showed that DNA methylation of the $\mathrm{CpG}$ island at the proximal promoter does not control KPNA7 expression after MZT, thus the 
silencing of KPNA7 expression after 16-cell embryonic stage is due to another mechanism.

After fertilization, the paternal genome undergoes spontaneous global demethylation before first cleavage, but the maternal genome retained the methylation status until the first cleavage and demethylation occurs passively with each cell division (Santos, et al. 2002). In cattle, the embryonic genome is activated at the 16-cell stage and completely activated in blastocyst stage embryos. The methylation status of the 5 , terminal region near the TSS of developmentally important genes was surveyed. All the genes including Oct4, Sox2, Nanog, Rex1 and Fgf4 experienced demethylation after fertilization and the expression of those gene were not accompanied by demethylation of their promoter region (Lan, et al. 2010). Therefore, the dynamic methylation changes in early embryonic development especially during MZT might not contribute to activation of gene transcription. In this case, the reduction of KPNA7 mRNA is explained by inactivation of transcription activity, however, on the other hand rapid mRNA degradation might be a reason for diminished the KPNA7 expression. Thus, we further tested the involvement of miRNA1296 in regulation of KPNA7 expression.

\section{miRNA binds to the coding region of bovine KPNA7}

To identify miRNAs that potentially targets KPNA7, full-length KPNA7 cDNA sequence were uploaded to the online algorithm "Microinspector" to predict binding sites (Figure 5). Four predicted MREs (miRNA recognition element) in KPNA7 ORF had the lowest predicted free energy $(-35.9,-33,-26$ and $-24.5 \mathrm{kcal} / \mathrm{mole})$ of hybridization with the cognate miRNA, which indicates a stable miRNA:mRNA interaction pair within 9 
nucleotide seed region at the 5' end of miRNA1296. The complementation between miRNA seed region and the mRNA requires a low degree of complementation in the 3' region of the miRNA to be functional, so the 5 ' end of the miRNA is a primary determinant of target specificity (Brodersen and Voinnet 2009). With a strong potential to form complementation duplex of miRNA1296 and KPNA7 mRNA, the functional role of miR1296 in regulation of KPNA7 was investigated further. In addition, the predicted binding sites were located within the coding region of KPNA7 instead of 3'UTR. This fashion of miRNA:mRNA recognition has recently been discovered in other developmentally important maternal factors such as NANOG, OCT4 and SOX2, which supports the augmented model that animal miRNAs can bind to target mRNAs beyond the 3'UTR region (Tay, et al. 2008).

\section{miR1296 is spatio-temporally regulated during development}

To determine if miRNA1296 is co-present with KPNA7 in oocytes, quantitative real-time PCR was performed. This miRNA is expressed predominantly in bovine fetal ovary, which is enriched with primordial oocytes arresting in meiosis prophase I stage (Figure 6a). Developmental stage real-time PCR results indicated that miRNA 1296 was negatively correlated to KPNA7 expression. The miRNA1296 increased and reached its peak level at the 8-cell stage embryo, which is coincident with the starting point of decline in KPNA7 (Figure 6b). Therefore, these data support the hypothesis that miRNA1296 acts as a functional physiological regulator of KPNA7 during early embryogenesis.

Targets of miRNA1296 have been studied and shown to be highly involved in essential DNA replication (Guiguen, et al. 1999). miRNA1296 showed low expression in prostate 
cancer cells during a rapid amplification stage. It targeted MCM2 mRNA in prostate cancer cells and over expression of miRA1296 resulted in a significant decrease in MCM2 mRNA, protein and movement of cells to the S-phase of the cell cycle. MCM2 protein is an essential DNA replication factor that is highly expressed in cancer cells as well as in oocyte and highly involved in resumption of meiosis in mammalian oocytes (Swiech, et al. 2007). Therefore, miRNA 1296 might be involved in other biological processes that, together with KPNA7, regulate oocyte and early embryo development.

\section{miRNA1296 specifically suppresses the expression of bovine KPNA7 through binding sites in coding region}

There is no endogenous expression of bovine KPNA7 or miRNA1296 in HEK293 cell line (data not shown). HEK293 cell was chosen in this study to confirm the specificity and binding of miRNA1296 to KPNA7. After co-transfection of expression vectors harboring miRNA1296 and KPNA7, a significant inhibition of KPNA7 protein expression was observed compared to cells transfected with KPNA7 alone (Figure 7a). This experiment was repeated three times and quantitative analysis of western blot data showed a significant reduction of KPNA7 protein in the co-transfection groups. These results demonstrate that bovine KPNA7 expression is regulated at the post-transcriptional level by miRNA1296. To test the specificity of the binding between miRNA1296 and KPNA7 mRNA, luciferase reporter assays were used. Four mutations in the predicted MRE in the coding region of KPNA7 were created to interfere with the binding of miRNA1296 and target sites (Figure 5). Mutations on predicted sites A, B and D failed to interfere with the recognition of miRNA1296 and KPNA7 as the luciferase activity of 
those mutation groups were as low as the treatment group with both wildtype KPNA7 ORF and miRNA1296. Luciferase activity was restored when a five-base mismatch was introduced on the MRE of predicted binding site $\mathrm{C}$, which indicated that this site is the primary binding site on KPNA7 ORF for miRNA1296 to recognize and interact (Figure 7b). These results indicated that the binding of miRNA1296 to KPNA7 ORF is specific and genuine.

The classic miRNA mediated post-transcriptional regulation of mRNAs was confined to the 3'UTR region of the transcripts (Bartel 2004). However, previous studies demonstrated the existence of many naturally occurring alternative binding sites of miRNA in mammalian cells (Mandke, et al. 2012, Tay, et al. 2008). During embryogenesis, miR-134, miR-296 and miR-470, in the presence of mouse embryonic stem cells, targeted the CDS of transcription factors Nanog, Oct4 and Sox2 in various combinations, leading to transcriptional and morphological changes in mouse embryonic stem cells (Tay, et al. 2008). In cattle, Nanog, Oct4 and Sox 2 are all oocyte-specific maternal factors governing early embryonic development by regulating the pluripotency of blastomeres (Goissis and Cibelli 2014, Kurosaka, et al. 2004). The present data, also showed similar targeting between miRNA 1296 and KPNA7 mRNA. Thus miRNA targeting the open reading frame of a developmentally important factors might be a characteristic feature in post-transcriptional regulation of the maternal effect factors.

\section{Conclusion}

In this study the mechanism controlling KPNA7 expression was explored. We found the oocyte restricted tissue-specific expression of bovine KPNA7 was regulated by DNA 
methylation at the proximal promoter and demethylation of three $\mathrm{CpG}$ sites was closely related to tissue specific expression of this gene. On the other hand, as a maternal effect gene KPNA7 is depleted during bovine early embryogenesis. It was found that miRNA1296 is involved in degradation of KPNA7 mRNA through a specific binding site on the coding region of KPNA7 mRNA. This study explored two aspects of epigenetic regulation of gene expression and discovered a distinctive controlling mechanism of tissue- and stage-specific expression of bovine KPNA7. 


\section{Reference}

Alizadeh Z, Kageyama S \& Aoki F 2005 Degradation of maternal mRNA in mouse embryos: selective degradation of specific mRNAs after fertilization. Molecular reproduction and development 72 281-290.

Bartel DP 2004 MicroRNAs: genomics, biogenesis, mechanism, and function. Cell 116 281-297.

Bazzini AA, Lee MT \& Giraldez AJ 2012 Ribosome profiling shows that miR-430 reduces translation before causing mRNA decay in zebrafish. Science (New York, NY) 336 233-237.

Borgel J, Guibert S, Li Y, Chiba H, Schübeler D, Sasaki H, Forné T \& Weber M 2010 Targets and dynamics of promoter DNA methylation during early mouse development. Nature Genetics 42 1093-1100.

Brodersen P \& Voinnet 02009 Revisiting the principles of microRNA target recognition and mode of action. Nature Reviews Molecular Cell Biology 10 141.

Canastar A, Logel J, Graw S, Finlay-Schultz J, Osborne C, Palionyte M, Drebing C, Plehaty M, Wilson L, Eyeson R \& Leonard S 2012 Promoter Methylation and Tissue-Specific Transcription of the $\alpha 7$ Nicotinic Receptor Gene, CHRNA7. Journal of Molecular Neuroscience 47 389-400.

Giraldez AJ, Mishima Y, Rihel J, Grocock RJ, Van Dongen S, Inoue K, Enright AJ \& Schier AF 2006 Zebrafish MiR-430 promotes deadenylation and clearance of maternal mRNAs. Science (New York, NY) 312 75-79.

Goissis MD \& Cibelli JB 2014 Functional Characterization of SOX2 in Bovine Preimplantation Embryos. Biology of reproduction 9030.

Guiguen Y, Baroiller JF, Ricordel MJ, Iseki K, McMeel OM, Martin SA \& Fostier A 1999 Involvement of estrogens in the process of sex differentiation in two fish species: the rainbow trout (Oncorhynchus mykiss) and a tilapia (Oreochromis niloticus). Molecular reproduction and development 54 154162.

Hattori N, Nishino K, Ko Y, Hattori N, Ohgane J, Tanaka S \& Shiota K 2004 Epigenetic control of mouse Oct-4 gene expression in embryonic stem cells and trophoblast stem cells. Journal of Biological Chemistry 279 17063-17069.

Hu J, Wang F, Yuan Y, Zhu X, Wang Y, Zhang Y, Kou Z, Wang S \& Gao S 2010 Novel importin-alpha family member Kpna7 is required for normal fertility and fecundity in the mouse. Journal of Biological Chemistry 285 3311333122.

Irizarry RA, Ladd-Acosta C, Wen B, Wu Z, Montano C, Onyango P, Cui H, Gabo K, Rongione M \& Webster M 2009 Genome-wide methylation analysis of human colon cancer reveals similar hypo-and hypermethylation at conserved tissue-specific CpG island shores. Nature Genetics 41178.

Itman C, Miyamoto Y, Young J, Jans D \& Loveland K. 2009. Nucleocytoplasmic transport as a driver of mammalian gametogenesis. In Seminars in cell \& developmental biology 5, pp. 607-619. 
Jones PA 1985 Effects of 5-azacytidine and its 2' -deoxyderivative on cell differentiation and DNA methylation. Pharmacology \& therapeutics 28 17-27.

Jones PA 2012 Functions of DNA methylation: islands, start sites, gene bodies and beyond. Nature reviews Genetics 13 484-492.

Jüttermann R, Li E \& Jaenisch R 1994 Toxicity of 5-aza-2'-deoxycytidine to mammalian cells is mediated primarily by covalent trapping of DNA methyltransferase rather than DNA demethylation. Proceedings of the National Academy of Sciences 91 11797-11801.

Kass SU, Landsberger N \& Wolffe AP 1997 DNA methylation directs a timedependent repression of transcription initiation. Current Biology 7 157-165.

Kobayashi H, Sakurai T, Imai M, Takahashi N, Fukuda A, Yayoi O, Sato S, Nakabayashi K, Hata K \& Sotomaru Y 2012 Contribution of intragenic DNA methylation in mouse gametic DNA methylomes to establish oocyte-specific heritable marks. PLoS Genetics 8 e1002440.

Kurosaka S, Eckardt S \& McLaughlin KJ 2004 Pluripotent lineage definition in bovine embryos by Oct4 transcript localization. Biol Reprod 71 1578-1582.

Lan J, Hua S, Zhang H, Song Y, Liu J \& Zhang Y 2010 Methylation patterns in 5 'terminal regions of pluripotency-related genes in bovine in vitro fertilized and cloned embryos. Journal of Genetics and Genomics 37 297-304.

Li L, Zheng P \& Dean J 2010 Maternal control of early mouse development. Development 137 859-870.

Lingenfelter BM, Tripurani SK, Tejomurtula J, Smith GW \& Yao J 2011 Molecular cloning and expression of bovine nucleoplasmin 2 (NPM2): a maternal effect gene regulated by miR-181a. Reprod Biol Endocrinol 940.

Lund E, Liu M, Hartley RS, Sheets MD \& Dahlberg JE 2009 Deadenylation of maternal mRNAs mediated by miR-427 in Xenopus laevis embryos. RNA (New York, NY) 15 2351-2363.

Maatouk DM, Kellam LD, Mann MRW, Lei H, Li E, Bartolomei MS \& Resnick JL 2006 DNA methylation is a primary mechanism for silencing postmigratory primordial germ cell genes in both germ cell and somatic cell lineages. Development 133 3411-3418.

Maeda C, Sato S, Hattori N, Tanaka S, Yagi S \& Shiota K 2008 DNA hypomethylation circuit of the mouse oocyte-specific histone H1foo gene in female germ cell lineage. Biology of reproduction 78 816-821.

Mandke P, Wyatt N, Fraser J, Bates B, Berberich SJ \& Markey MP 2012 MicroRNA-34a Modulates MDM4 Expression via a Target Site in the Open Reading Frame. PLoS ONE 7 e42034.

Messerschmidt DM. 2012. Should I stay or should I go: Protection and maintenance of DNA methylation at imprinted genes. In Epigenetics 9.

Nishino K, Hattori N, Tanaka S \& Shiota K 2004 DNA methylation-mediated control of Sry gene expression in mouse gonadal development. Journal of Biological Chemistry 279 22306-22313.

Panning B \& Jaenisch R 1996 DNA hypomethylation can activate Xist expression and silence X-linked genes. Genes \& Development 10 1991-2002. 
Paynton BV, Rempel R \& Bachvarova R 1988 Changes in state of adenylation and time course of degradation of maternal mRNAs during oocyte maturation and early embryonic development in the mouse. Developmental biology 129 304-314.

Ramachandra RK, Salem M, Gahr S, Rexroad CE \& Yao J 2008 Cloning and characterization of microRNAs from rainbow trout (Oncorhynchus mykiss): their expression during early embryonic development. BMC Dev Biol 841.

Rao X, Evans J, Chae H, Pilrose J, Kim S, Yan P, Huang R, Lai H, Lin H \& Liu Y 2013 CpG island shore methylation regulates caveolin-1 expression in breast cancer. Oncogene 32 4519-4528.

Sagata N, Watanabe N, Woude GFV \& Ikawa Y 1989 The c-mos proto-oncogene product is a cytostatic factor responsible for meiotic arrest in vertebrate eggs.

Santos F, Hendrich B, Reik W \& Dean W 2002 Dynamic reprogramming of DNA methylation in the early mouse embryo. Developmental biology 241 172-182.

Shiota K 2004 DNA methylation profiles of CpG islands for cellular differentiation and development in mammals. Cytogenetic and genome research 105325 334.

Smallwood SA, Tomizawa S, Krueger F, Ruf N, Carli N, Segonds-Pichon A, Sato S, Hata K, Andrews SR \& Kelsey G 2011 Dynamic CpG island methylation landscape in oocytes and preimplantation embryos. Nature Publishing Group 43 811-814.

Song F, Mahmood S, Ghosh S, Liang P, Smiraglia DJ, Nagase H \& Held WA 2009 Tissue specific differentially methylated regions (TDMR): Changes in DNA methylation during development. Genomics 93 130-139.

Swiech L, Kisiel K, Czolowska R, Zientarski M \& Borsuk E 2007 Accumulation and dynamics of proteins of the MCM family during mouse oogenesis and the first embryonic cell cycle. International Journal of Developmental Biology $\mathbf{5 1}$ 283.

Tay Y, Zhang J, Thomson AM, Lim B \& Rigoutsos I 2008 MicroRNAs to Nanog, Oct4 and Sox2 coding regions modulate embryonic stem cell differentiation. Nature 455 1124-1128.

Tejomurtula J, Lee K, Tripurani SK, Smith GW \& Yao J 2009 Role of Importin Alpha8, a New Member of the Importin Alpha Family of Nuclear Transport Proteins, in Early Embryonic Development in Cattle. Biology of reproduction 81 333-342.

Tripurani SK, Lee K, Wee G, Smith GW \& Yao J 2011 MicroRNA-196a regulates bovine newborn ovary homeobox gene (NOBOX) expression during early embryogenesis. BMC Developmental Biology 1125.

Tripurani SK, Wee G, Lee K, Smith GW, Wang L \& Jianboyao 2013 MicroRNA-212 post-transcriptionally regulates oocyte-specific basic-helix-loop-helix transcription factor, factor in the germline alpha (FIGLA), during bovine early embryogenesis. PLOS ONE 8 e76114.

Tripurani SK, Xiao C, Salem M \& Yao J 2010 Cloning and analysis of fetal ovary microRNAs in cattle. Animal reproduction science 120 16-22. 
Wang L, Tripurani SK, Wanna W, Rexroad CE \& Yao J 2013 Cloning and characterization of a novel oocyte-specific gene encoding an F-Box protein in rainbow trout (Oncorhynchus mykiss). Reproductive Biology and Endocrinology 1186.

Wang X, Park KE, Koser S, Liu S, Magnani L \& Cabot RA 2011 KPNA7, an oocyteand embryo-specific karyopherin $\alpha$ subtype, is required for porcine embryo development. Reproduction, Fertility and Development.

Zhang X, Wu M, Xiao H, Lee M, Levin L, Leung Y \& Ho S 2010 Methylation of a single intronic $\mathrm{CpG}$ mediates expression silencing of the PMP24 gene in prostate cancer. The Prostate 70 765-776.

Figure Legend

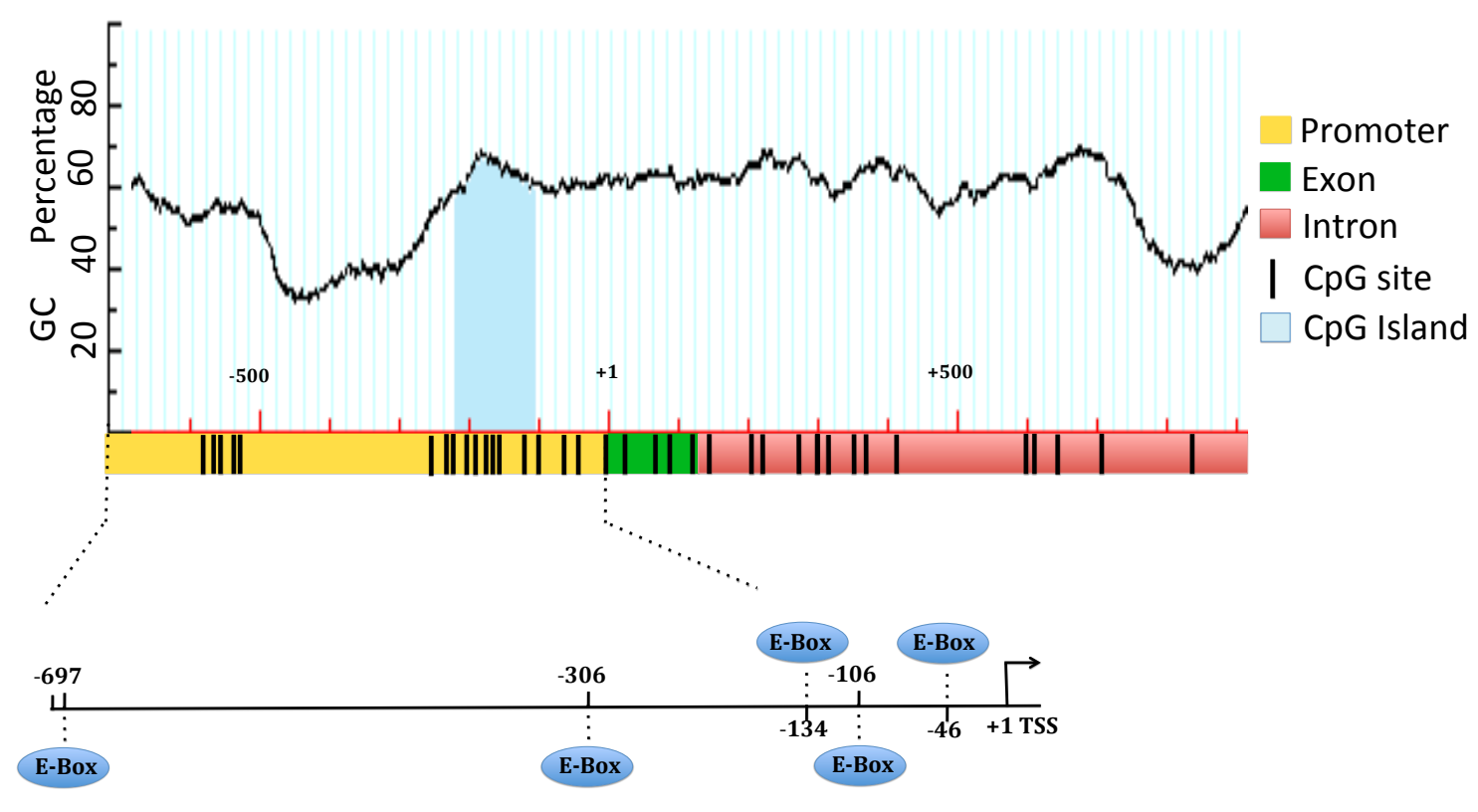

Figure 1. CpG structure on proximal promoter of KPNA7. Putative cis acting elements were predicted and labeled according to their location on the proximal promoter of KPNA7. 
GV oocytes

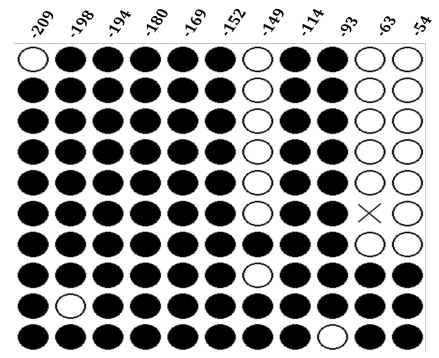

Heart

0909090009 0989690009 08089800980 0886880088 8886880088 8888888088 8888888088 8888888888 8888888888 0000000000

Brain

0909090090 0898989090 8088088880 8088088889 8088088888 8088080868 0080809008 080008000 08989898980 08080808080
Lung

○9090009990 $88 \bigcirc 98 \bigcirc 09898$ 0808909068 0009809008 0080808008 0800980608 0880808080 0880808080 0888808088 08888808808

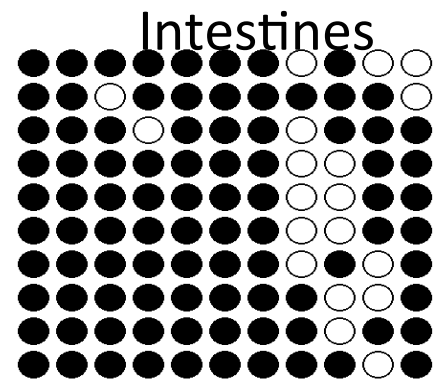

Muscle

○९999९९९98 0008900098 0808800088 0000000000 0808980808 0000000900 000600000 000008000 8888808888 0808080808

Spleen

098989009 0890808008 ○9809890989  0880880080

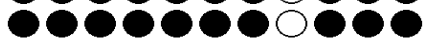
0909090909 0808080800 8088086808 0000000000

Figure 2. Differential methylated region on proximal promoter of Kpna7. Methylation status of leven $\mathrm{CpG}$ sites closest to TSS were examined across GV oocytes and somatic tissues. Three tissue-specific differential methylated sites $(-54,-63$ and -149$)$ were detected. Close circle indicated methylated sites; open circle indicated demethylated sites. 


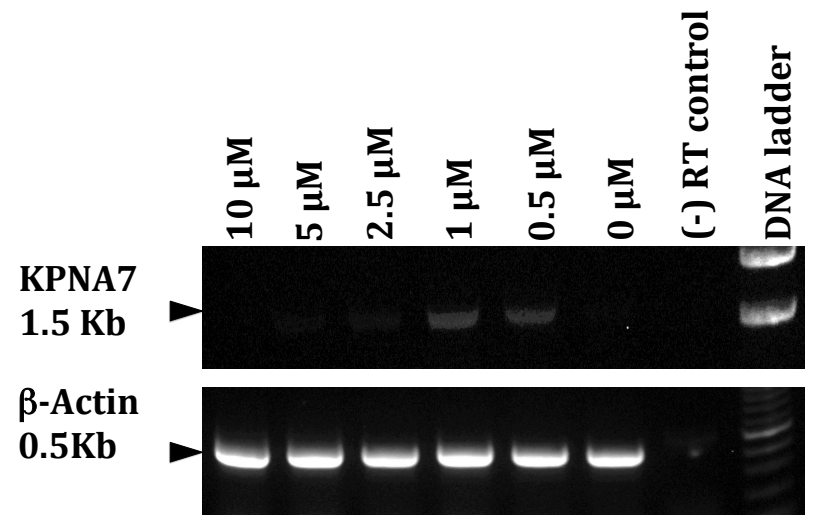

Figure 3a. 5-aza treatment of CCL-22 cell reintroduce KPNA7 expression. $\beta$-actin is used as a control. The entire ORF of KPNA7 was amplified as a $1.5 \mathrm{~K}$ fragment, indicated by the upper arrowhead. 


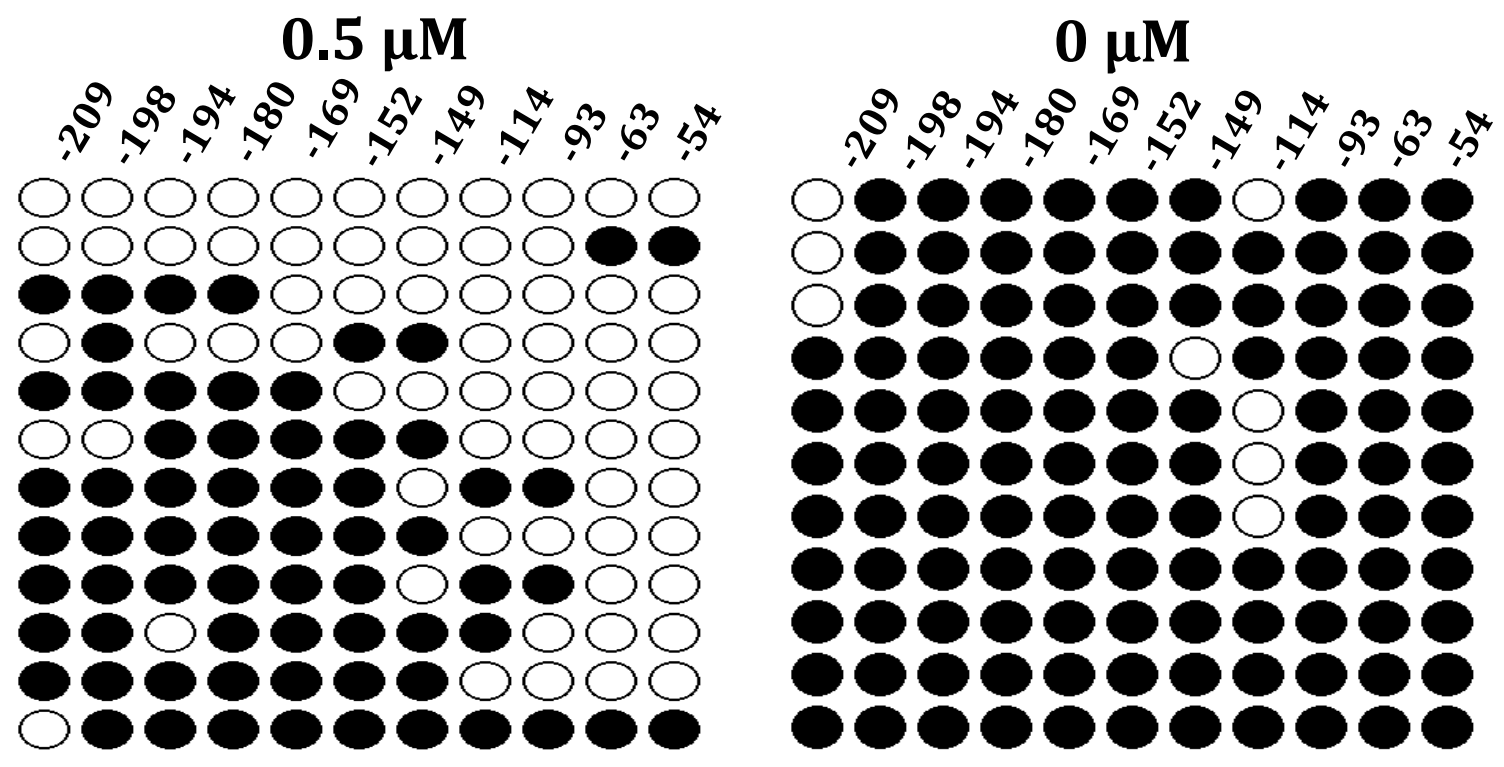

Figure 3b. 5-aza treatment of CCL-22 cell reintroduce KPNA7 expression by demethylation of CpG sites on the proximal promoter of KPNA7. Two sites (-54 and 63 ), which are highly demethylated in the treated cells, are the sites related to KPNA7 tissue-specific expression. Close circles indicate dmethylated sites; open circles indicate demethylated sites. 
16-cell stage

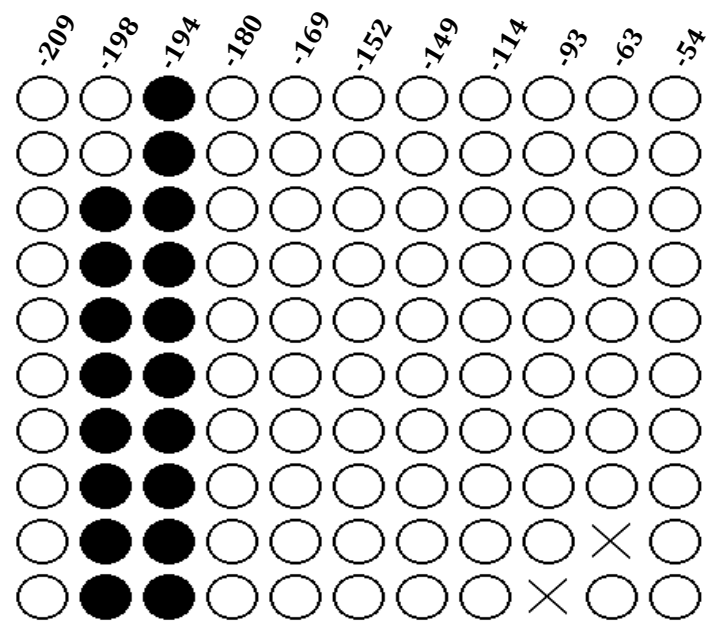

Blastocyst stage

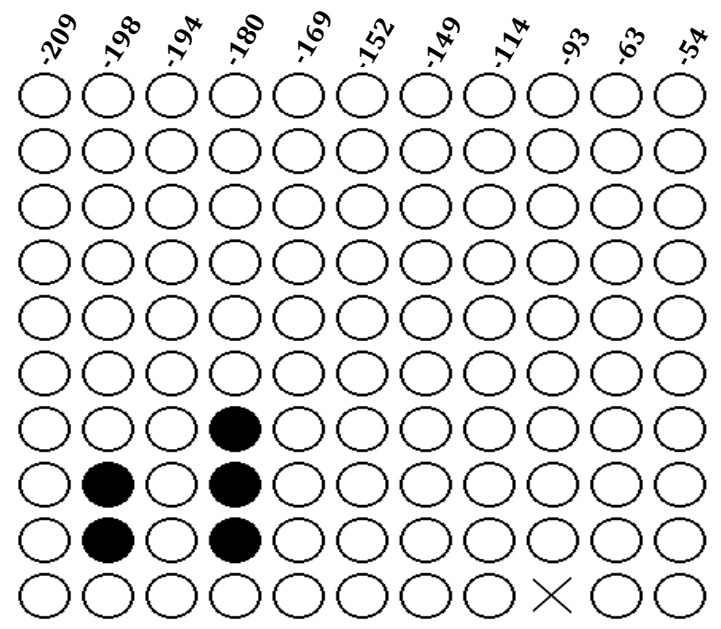

Figure 4. DNA demethylation on the proximal promoter does not activate KPNA7 expression during early embryonic development in cattle. Close circles indicate dmethylated sites; open circles indicate demethylated sites. 


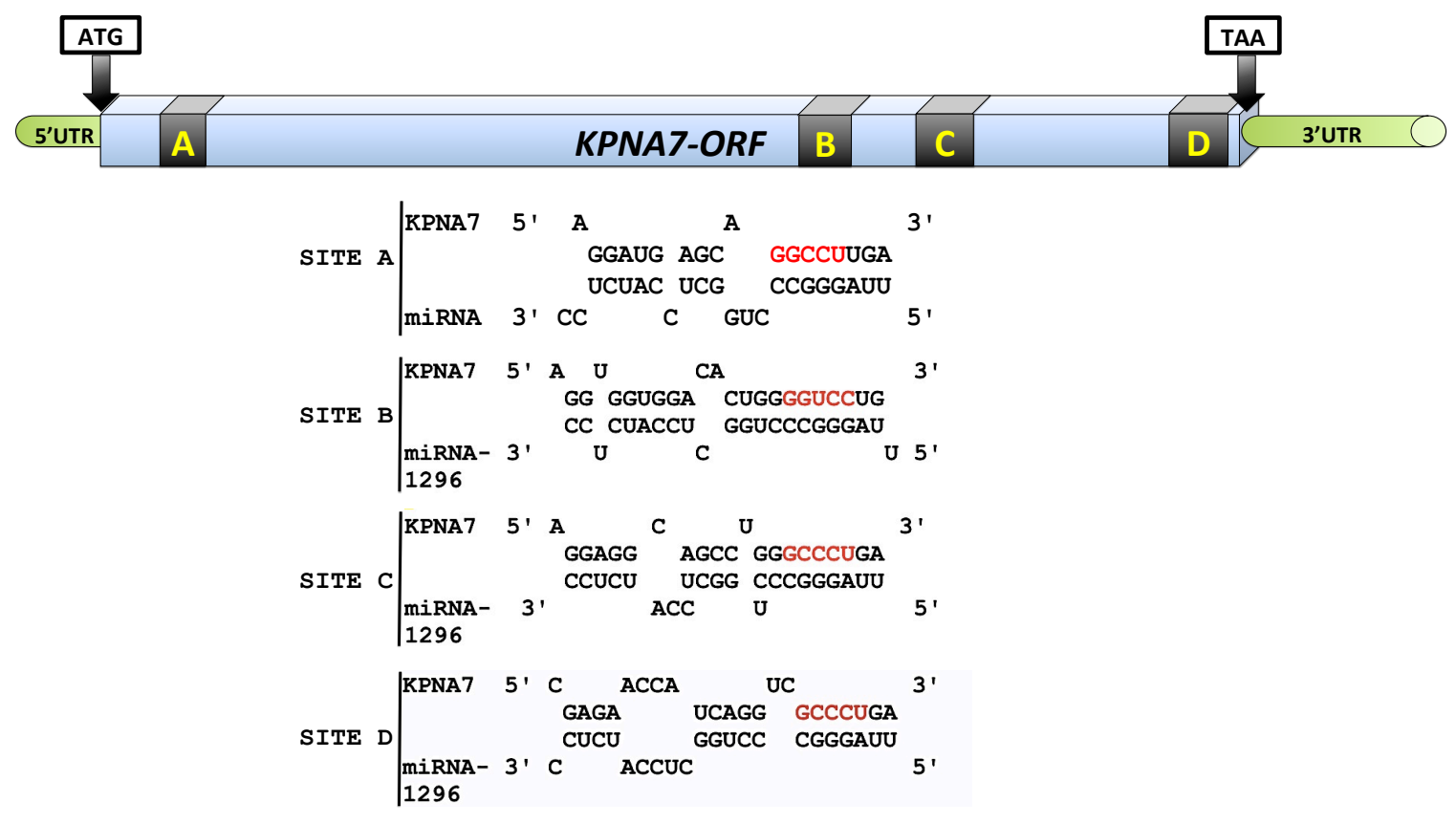

Figure 5. Prediction of miRNA1296 binding sites in the ORF of bovine KPNA7 mRNA. Green bars indicate the 5' and 3' UTR regions of KPNA7; blue bar represents the KPNA7 ORF region; black boxes indicate putative miRNA:mRNA binding sites; red letters show the sites of point mutations. 


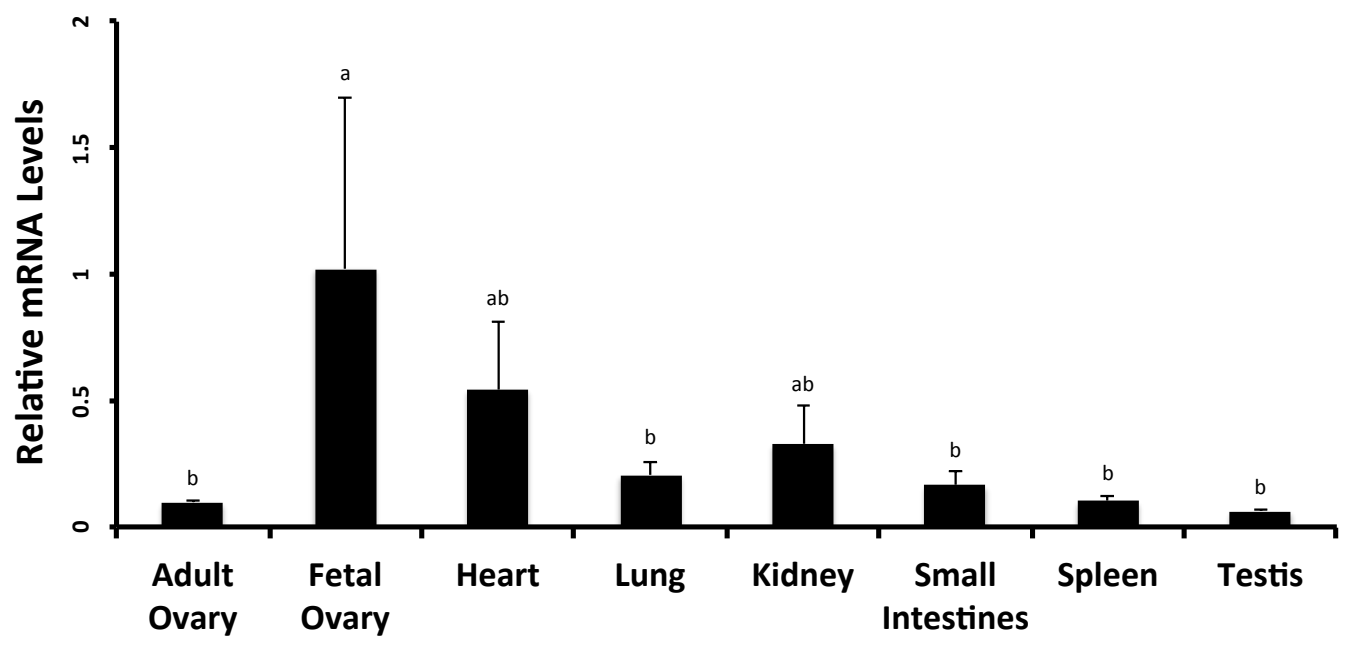

Figure 6a Tissue distribution of miR-1296 analyzed by quantitative real-time PCR. Quantity of miRNA-1296 was normalized to abundance of RPS18 mRNA and abundance expressed as relative mRNA levels $(n=4$ per tissue; mean \pm SEM). Different letters indicate statistical difference $(\mathrm{P}<0.05)$. 


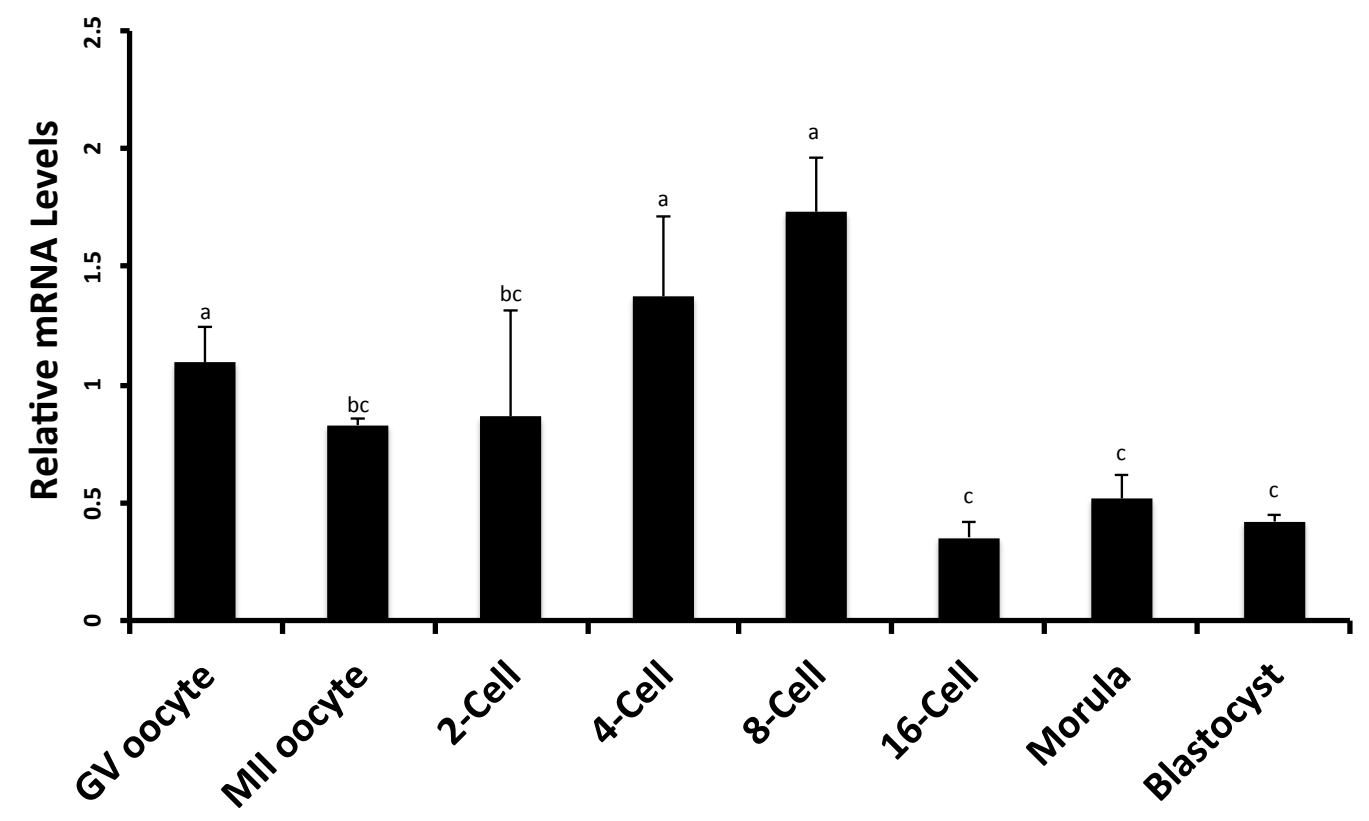

Figure 6b. Relative abundance of miR-1296 in bovine oocytes and in vitro produced bovine early embryos ( $n=4$ pools of five oocytes/embryos each). Quantity of miRNA was normalized relative to abundance of miR-125b. Different letters indicate statistical difference $(\mathrm{P}<0.05)$. 

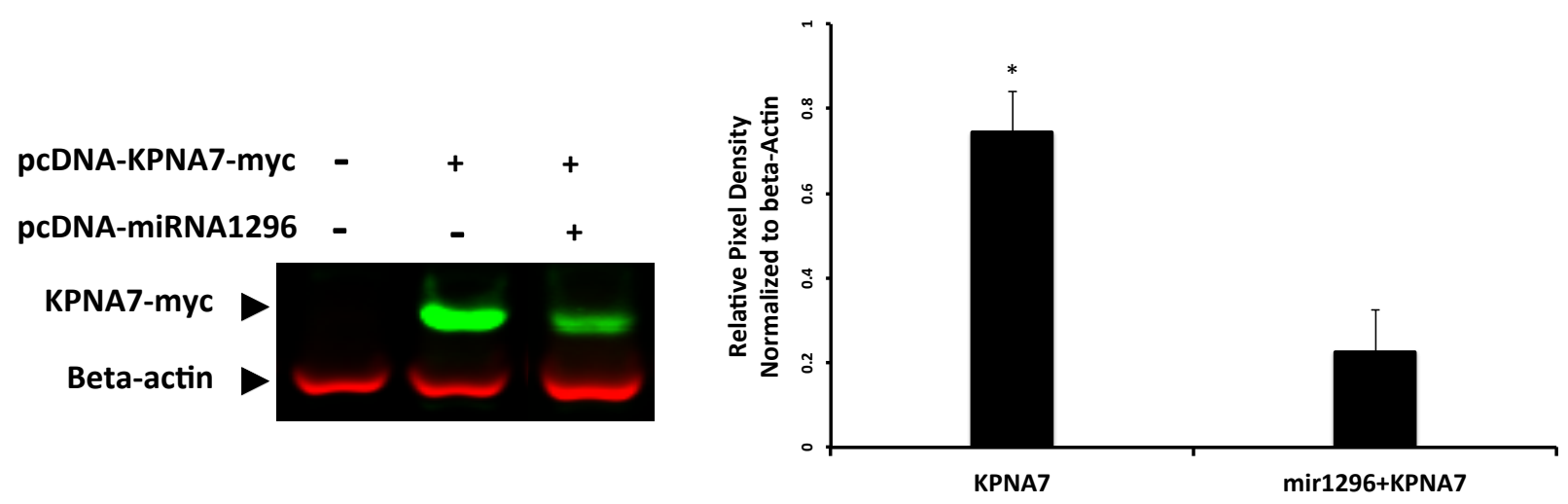

Figure 7a. miRNA1296 specifically suppresses the expression of bovine KPNA7 protein in citro in HEK293 cells. Left panel is the representative western blot showing specific suppression of bovine KPNA7 by miR-1296a in HEK293 cells. $\beta$-Actin was used as loading control. The experiment was repeated three times. ImageJ software was used to determine the densitometry of KPNA7 as well as $\beta$-actin protein in each sample. Abundance of KPNA7 protein in was normalized relative to abundance of $\beta$-actin protein (right panel). Data are expressed as mean relative pixel density $(n=3$ mean \pm SEM). Asterisk indicates statistical difference $(\mathrm{P}<0.05)$. 


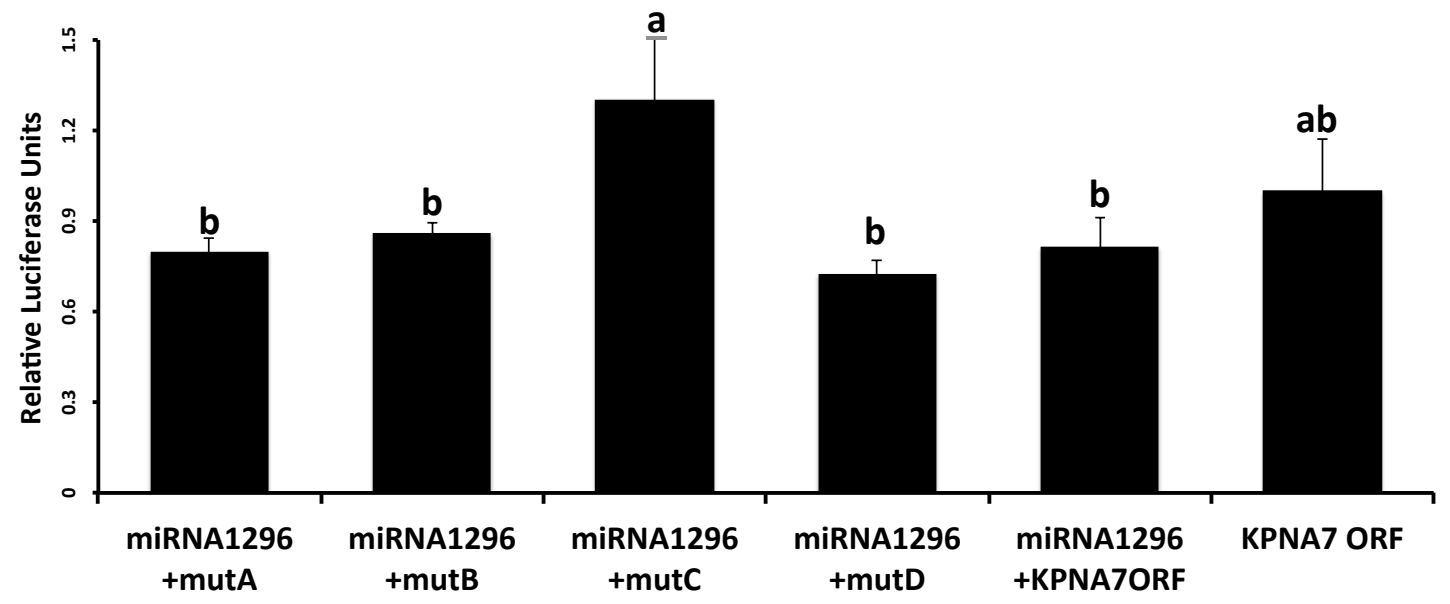

Figure 7b. miRNA1296 specifically suppresses the expression of bovine KPNA7 protein through site $\mathrm{C}$ in coding region. Repression of luciferase reporter gene activity by miR1296 was abolished when the binding site $\mathrm{C}$ was mutated. Data is presented as relative firefly luciferase units (RLUs). Each group represents the mean \pm SEM of 12 wells for an experiment repeated three times with similar results. Different letters indicate statistical difference $(\mathrm{P}<0.05)$. 



\section{Chapter 3:}

Specific gene-regulation networks of KPNA7 during the pre-implantation development of the bovine embryo as revealed by deep sequencing

Lei Wang ${ }^{1}$, Jian Wang ${ }^{1}$, Gabbine Wee ${ }^{2}$, George W. Smith ${ }^{2}$, Jianbo Yao ${ }^{1}$

${ }^{1}$ Laboratory of Animal Biotechnology and Genomics, Division of Animal and Nutritional Sciences, West Virginia University, Morgantown, WV 26506.

${ }^{2}$ Laboratory of Mammalian Reproductive Biology and Genomics, Departments of Animal Science and Physiology, Michigan State University, East Lansing, Michigan 48824.

Key Words: KPNA7, siRNA, oocyte maturation, early embryo development, maternal, cattle

* Corresponding author:

Jianbo Yao, Ph.D.

Tel: (304) 293-1948

e-mail: jianbo.yao@mail.wvu.edu 


\begin{abstract}
Oocyte-specific genes play critical roles in oogenesis, folliculogenesis and early embryonic development. A novel member of the KPNA family (named KPNA7) was previously identified, through analysis of expressed sequence tags (ESTs) from a bovine oocyte cDNA library and a requirement of the oocyte-specific KPNA7 for bovine embryogenesis was demonstrated. Given that KPNA7 is highly expressed in oocyte, a knockdown model system whereby KPNA7 siRNA was microinjected into cumulusenclosed GV oocytes was developed to examine the functional role of KPNA7 in meiotic maturation, cumulus expansion and embryonic development. Knockdown of KPNA7 protein during in vitro maturation lead to reduction of matured oocytes, embryo cleavage rate and development to 8-16 cell stage and no development of blastocyst compared to controls. To further explore the functional network controlled by KPNA7, cDNA was synthesized from 8-cell stage embryos and subjected to deep sequencing to compare the transcriptome change between knockdown and control groups. A total of 1761 genes showed differential expression pattern. Gene set enrichment analysis revealed that several Gene Ontology terms were enriched $(\mathrm{FDR}<0.05)$ with differentially expressed genes involved in embryo development. In conclusion, these results support the hypothesis that KPNA7 as a critical oocyte-specific protein plays important roles through functional networks that regulate oocyte maturation and subsequent embryonic development.
\end{abstract}




\section{Introduction}

Early embryonic loss is a major contributing factor to infertility in livestock species (Diskin, et al. 2011). A growing body of evidence indicates that the oocyte plays an active role in regulation of key aspects of the reproductive process required for fertility. Oocyte-specific genes are crucial functional mediators of oocyte and early embryonic development (Ruddock-D'Cruz, et al. 2007). Nuclear proteins, including oocyte-specific chromatin remodeling factors and (or) transcription factors, are required for key regulatory events characteristic of oocyte growth and development, meiotic maturation and initial cleavage divisions following fertilization culminating in embryonic genome activation (Bettegowda, et al. 2008, Betthauser, et al. 2006, Mathe, et al. 2000). Thus, regulated transport of nuclear proteins into the nucleus through the nuclear pores is a vital highly regulated event in oocytes. KPNAs are the major cargo carriers from the cytoplasm into the nucleus.

Seven KPNAs have been identified from several model species and the functional roles of them have been studied intensively. Previous studies showed that during mouse spermatogenesis, neural lineage specification and maintenance of embryonic stem cell, different KPNA proteins have distinct properties in terms of NLS recognition and nuclear transport efficiency for specific cargo proteins (Hogarth, et al. 2006, Yasuhara, et al. 2007, Yasuhara, et al. 2013, Young, et al. 2011). An in vivo study of the effects of RNAiinduced knockdown of specific KPNA proteins in cultured cells supported the notion that each KPNA is part of specific cellular pathways (Geles and Adam 2001). However, the mechanisms that control nuclear transport in oocytes and early embryos of chromatin 
remodeling factors and transcription factors critical to developmentally regulated processes are not completely understood.

Among seven KPNA family members, only Kpna7 is found preferentially expressed in bovine, porcine and murine oocytes (Hu, et al. 2010a, Tejomurtula, et al. 2009, Wang, et al. 2011). In bovine and porcine studies, Kpna7 showed a pattern of a maternal effect factor, which is highly expressed in germinal vesicle (GV) oocytes through early-stage embryos, but is barely detectable in morula and blastocyst stage embryos after the maternal to embryonic transmission. Knocking down of Kpna7 led to arrested embryonic development (Tejomurtula, et al. 2009, Wang, et al. 2011). Knockout of Kpna7 in female mice caused a reduction in fecundity and an imbalance of by sexes inducing preferential fetal lethality. The induced abnormalities of gene expression (Dppa2, Dppa4 and Piwil2) and epigenetic modifications (down-regulation of histone H3K27me3) were part of the Kpna7 knock out effects (Hu, et al. 2010a). These results indicated that KPNA7 is essential to early embryogenesis. However, the cellular mechanisms maintained and regulated by KPNA7 have not been explored extensively to enable an understanding of genes regulated functionally of by KPNA7 during normal embryogenesis or genes deregulated in KPNA7-deficient embryos.

Differential gene expression has gained increasing attention as a mechanism underlying the phenotypic variance observed from early embryos with different quality, different origin or different cell types (Driver, et al. 2012, Ozawa, et al. 2012, Xie, et al. 2010). The technique of RNA-seq is a powerful new tool that allows whole genome cross 
analysis of the entire transcriptome and is being applied to abovementioned developmental biology researches. The traditional method of microarrays requires the generation of probes based on known gene information and has inevitable system background. Compared to microarrays, RNA-seq has low noise, higher capacity of screening and most importantly it led to discovery of novel transcripts or novel alternative splicing genes (Wang, et al. 2009). Therefore, in this study, RNA-seq is a perfect fit for the goal to search for the functional network controlled by KPNA7 during early embryonic development.

\section{Material and Methods}

\section{In vitro maturation, fertilization and embryo culture}

In vitro maturation (IVM), in vitro fertilization (IVF) and embryo culture (IVC) were performed as previously described (Lee, et al. 2014). Briefly, cumulus-oocyte-complexes (COCs) were collected from ovaries obtained from local slaughterhouse. COCs were cultured in four-well dishes (NUNC) containing maturation medium (Medium-199, 10\% FBS (Gibco-BRL, Grand Island, NY), $1 \mathrm{IU} / \mathrm{ml} \mathrm{FSH,} 5 \mathrm{IU} / \mathrm{ml} \mathrm{LH}$ and $1 \mu \mathrm{g} / \mathrm{ml}$ estradiol17ß. To arrest meiotic maturation and allow sufficient time for KPNA7 knockdown to occur, COCs were incubated initially for $48 \mathrm{~h}$ in maturation medium supplemented with $50 \mu \mathrm{M}$ S-roscovitine (Calbiochem, La Jolla, CA). Then COCs were washed several times with fresh maturation medium and incubated for $24 \mathrm{~h}$ in maturation medium minus Sroscovitine at $38.5^{\circ} \mathrm{C}$ under $5 \% \mathrm{CO} 2$ in humidified air. Oocytes in matured COCs (50 COCs/well) were fertilized with spermatozoa purified from frozen-thawed semen by Percoll gradient. After $20 \mathrm{~h}$ at $38.5^{\circ} \mathrm{C}$ under $5 \% \mathrm{CO} 2$ in humidified air, putative zygotes 
were stripped of cumulus cells by vortexing for $5 \mathrm{~min}$ and placed in potassium simplex optimization medium (KSOM; Specialty Media, Phillipsburg, NJ) supplemented with $0.3 \%$ BSA. Presumptive zygotes were cultured at $38.5^{\circ} \mathrm{C}$ under $5 \% \mathrm{CO} 2$ in humidified air. At $72 \mathrm{~h}$ post insemination (hpi), 8-16 cell embryos were removed, washed and incubated in fresh KSOM with $0.3 \%$ BSA and $10 \%$ fetal bovine serum until $\mathrm{d} 7$.

\section{Microinjection of siRNA into COCs}

Four interfering (siRNA) species targeting KPNA7 mRNA were designed using siRNA target finder (http://www.ambion.com/techlib/misc/siRNA finder.). KPNA7 siRNA cocktail (species 2 and 4,25 $\mu \mathrm{M}$ concentration) was microinjected into COC GV stage oocytes ( $\mathrm{n}=30$ oocytes injected per treatment; $\mathrm{n}=6$ replicates) using an inverted Nikon microscope (Mager Scientific, Dexter, MI) equipped with a micromanipulator system (Narishige International, Long Island, NY). Control oocytes were uninjected, injected with similar volume of water or injected with $25 \mathrm{nM}$ negative control siRNA (nonspecific; Ambion universal control \#1). Efficiency of KPNA7 siRNA in ablation of KPNA7 mRNA was determined by real-time PCR $(n=6$ pools of 10 oocytes per treatment) of denuded oocytes collected $72 \mathrm{~h}$ after siRNA microinjection.

\section{Effect of oocyte KPNA7 ablation on oocyte maturation and embryonic development}

After injection, all oocytes were cultured in the presence of $50 \mu \mathrm{M}$ S-roscovitine for $48 \mathrm{~h}$ and then subjected to meiotic maturation as described above. At the end of oocyte maturation, COCs were classified into one of three categories based on degree of cumulus expansion (non expanded, partially expanded and fully expanded). Then oocytes 
were removed from surrounding cumulus cells and treated with $4 \mu \mathrm{g} / \mathrm{ml}$ Hoechst 33342 dye for $20 \mathrm{~min}$. Denuded oocytes were washed, placed on a microscope slide and covered lightly with a cover glass. Meiotic phase (metaphase I, anaphase I, telophase I and metaphase II) of each oocyte was determined under a fluorescent microscope (Mager Scientific, Dexter, MI). Effects of oocyte KPNA7 ablation on early cleavage, total cleavage rates, development to $8-16$ cell and blastocyst stages were determined at $30 \mathrm{~h}$, $48 \mathrm{~h}, 72 \mathrm{~h}$ and $7 \mathrm{~d}$ post insemination, respectively. For RNA-seq 8-cell stage embryo samples, five fertilized presumptive zygotes were collected $16 \mathrm{~h}$ after sperm insemination and uninjected or injected with negative control siRNA or KPNA7 siRNA cocktail. After microinjection, injected zygotes were cultured in IVC medium and early 8-cell embryos were collected 52h after sperm insemination, placed in $2 \mu 1 \mathrm{PBS}$ and store $-70^{\circ} \mathrm{C}$ until use.

\section{cDNA synthesis and library preparation for RNA-seq}

Five 8-cell stage injected and uninjected embryos were pooled with 3 replications. RNA from these embryos was isolated and subsequently converted to cDNA with linear amplification using Ovation ${ }^{\circledR}$ RNA-Seq System V2 according to manufacturer's instruction. cDNA samples were stored in $-20^{\circ} \mathrm{C}$ and sent to UIUC core sequencing facility (Urbana, IL). Quality controls were performed on cDNA samples using bioanalyzer. Traces of each cDNA were measured and all the samples had around 1000 ng of DNA with a size range from $100 \mathrm{bp}$ to $1 \mathrm{~kb}$. RNA-seq libraries were prepared with Truseq DNA sample prep kit (Illumina, CA) using 500 ng of cDNA as input and final amplification was done for 6 cycles. Each library (in total 6 libraries) were labeled with 
distinctive barcode and quantitated by qPCR and sequenced on one lane for 101 cycles from one end of the cDNA fragments on a HiSeq2000 sequencer.

\section{Mapping Reads to the Reference Genome}

Quality of RNA-seq readings were checked using FastQC (Andrews 2012). Adaptor sequences were trimmed and reads with Phred quality below 30 were removed. Readings that were shorter than 50 bases were discarded. The pre-built Bos Taurus ENSEMBL UMD3.1 index was downloaded from the TopHat homepage and used as the reference genome. Then the clean reads were mapped to the Bovine Taurus reference genome using TopHat (v2.0.4) (Trapnell, et al. 2009). Briefly, using a two step mapping processes, TopHat first uses Bowtie2 to align reads that are directly mapped to the genome (with no gaps) (Langmead and Salzberg 2012). It then determines the possible location of gaps in the alignment based on canonical and non-canonical splice sites flanking the aligned reads. Finally, it uses gapped alignments to align the reads that were not aligned by Bowtie 2 in the first step. A maximum of 2 mismatches were allowed and reads that uniquely mapped to the genome were chosen.

\section{Assembly of Transcripts and Estimation of Abundance}

The resulting alignments were used to reconstruct transcript models using Cufflinks (v2.0.2). This program uses graph theory to find a parsimonious set of transcript models that satisfy with the alignments and also estimate transcript and gene expression (fragments per kilobase exon per million mapped fragments, or FPKM) by optimizing a likelihood function containing transcript abundances as parameters. In addition, the tool 
Cuffmerge was used for merging together each of the assemblies with the reference bovine annotation file in order to combine novel isoforms with known isoforms. This procedure maximizes the overall quality of the final assembly. Differential accumulation of transcripts between KPNA7 gene knock-down and negative control bovine embryos was tested by the Cuffdiff program within Cufflinks. Cuffdiff was run requiring a minimum alignment count of 10 and the upperquartile-norm option, which can improve robustness of differential accumulation calls for less abundant transcripts.

\section{Functional Annotation of DEGs}

The bovine Ensembl gene IDs were converted to GO (Gene Ontology) term ID by using BioMart provide by Ensembl. The GO IDs of DEGs were inputed into the BGI WEGO program and GO annotations were plotted (http://wego.genomics.org.cn). Functional categories with FDR $<0.05$ were considered significant.

\section{Results}

\section{Validation of KPNA7 siRNA}

To evaluate the efficiency of KPNA knockdown, we tested the efficacy of 4 KPNA7 siRNA species during meiotic maturation. After siRNA injection, oocytes were incubated in S-roscovitine, a chemical reagent that maintains the GV arrest of oocyte, for $48 \mathrm{~h}$ before changing the culture condition to IVM procedure. This incubation is tested and proved to block the resumption of meiosis in the oocytes, which is helpful for developing the siRNA knockdown effect (Lee, et al. 2014). Four siRNA species were microinjected into COCs following the method descripted above and two siRNAs (siRNA 2 and siRNA 
4) were able to reduce KPNA7 mRNA abundance by over $95 \%$ revealed by real-time PCR during meiotic maturation (Figure 1).

\section{KPNA7 deficiency compromised maturation of bovine COCs}

To test if KPNA7 knockdown affect the maturation of bovine oocyte, siRNA2 and 4 were microinject into COCs. Incubation with S-roscovitine was also performed in this experiment to ensure maximum performance of siRNAs. After 24h of IVM procedure, the percentage of oocytes with expanded cumulus cells was not different compared to sham injection and non-injection groups (Figure 2a). However, the number of oocytes harboring $1^{\text {st }}$ polar body was significantly reduced in injection groups of siRNA2 and 4 (Figure 2b). Protrusion of $1^{\text {st }}$ polar body is a marker for metaphase II stage indicating oocyte maturation. These results indicated that KPNA7 knockdown, although it did not affect cumulus expansion, compromised the competency of oocytes to reach mature stage in vitro.

\section{KPNA deficiency in COCs inhibited subsequent early embryonic development}

In mammalian in vitro embryo culture system, it is well known that oocyte maturation is prerequisite for and highly related to subsequent development of embryos. As observed in Figure 2, knockdown of KPNA7 inhibited oocyte maturation to some extent, but still allowed some oocytes to reach metaphase II stage. If fertilized, those matured oocyte with deficient KPNA7 might perform poorly in subsequent embryo development due to attenuated genetic profile caused by the KPNA7 knockdown. Thus, KPNA7 siRNAinjected and control groups were fertilized with bovine sperm. After fertilization, first 
round of cleavage were significantly reduced (Figure 3a.), moreover, after $72 \mathrm{~h}$ IVC a significant decrease in the percentage of embryos reached 8-16 cell stage was observed (60\% vs $35 \%$, control vs injected)(Figure $3 \mathrm{~b}$ ); even more extreme negative effects were noted after 7 days of IVC when $0 \%$ of injected oocytes were developed into blastocyst stage embryos (Figure 3c). Thus the data showed that KPNA7 deficiency in bovine oocytes led to retardation of early embryonic development.

\section{KPNA7 deficiency led to differential expression of thousand of genes}

To provide a comprehensive understanding of knockdown effects of KPNA7 on early bovine embryos, RNA samples from injected and non-injected 8-cell stage embryos were collected and deep sequenced. A total of 194,118,117 reads were generated from six samples (3 injection vs 3 non-injection) with 100-bp read length (Figure 4.). The number of sequences from each sample ranged from 27.0 to 37.2 million. After removal of ambiguous nucleotides and low-quality sequences (Phred quality scores $<30$ ), the cleaned sample ranged from 25.3 to 35.6 million and a total of $178,963,589$ cleaned reads $(92.1 \%)$ were harvested for further analysis. Cleaned reads were then mapped to bovine genome reference sequences and the mapping ratio ranged from $75.3 \%$ to $88.0 \%$ showing the stability and consistency of sampling, library preparation and sequencing (Table 1.). A total of 1761 genes were found to be at least a 2-fold expression difference, among them 1092 and 669 genes were found to be up and down regulated by KPNA7 knockdown. An M-A plot was generated to display the $\log 2$ fold change (the $\log 2$ ratio of expression levels for each gene between the two experimental groups) against the average $\log 2$ expression (the overall average expression for each gene across the two 
experimental groups). Most of the genes showed a fold change close to 0 (black dots), and differentially expressed genes were shown in red dots (Figure 5).

\section{Gene Set Enrichment Analysis: Evaluation of Different Gene Ontology Terms}

To gain insight into the biological processes that could be regulated differentially between injected and non-injected embryos, a gene set enrichment analysis was performed. Differentially expressed genes were analyzed and separated into different catalogs as cellular components, molecular functions and biological processes (Figure 6.). Several gene ontology terms gathered more than $10 \%$ of total differentially expressed genes, including anatomical structure formation (GO:0048646), Organelle part (GO:0044422), Binding (GO:0005488), Catalytic (GO:0003824), Biological regulation (GO:0065007), Cellular component regulation (GO:0009987), Localization (GO:0051179), Multicellular organismal processes (GO:0032501), Pigmentation (GO:0043473) and Response to stimulus (GO:0050896). Especially, more than 10\% of total differentially expressed genes were enriched in term GO:0032991 (macromolecular complex) representing a stable assembly of two or more macromolecules and constituent parts that function together, which indicates that KPNA7 knockdown affected the protein-protein interaction capacity of embryos. Terms such as Localization and Cellular component regulation are all closely related to the KPNA7 transportation function.

\section{Discussion}

The finely orchestrated development and maturation of the oocytes has been the focus of studies in which essential genes have been identified (Rodriguez and Farin 2004). Each 
one of these oocyte specific genes is essential for folliculogenesis and early embryo development, Nonetheless, rather than forming a direct linear relationship, these genes might form complex networks. Using knock-out mice models of Nobox, Sohlh1, Sohlh2, Figla and Lhx8 etc. (Zheng and Dean 2007a), it was revealed that these oocyte specific nuclear proteins formed networks in which they regulate the expression of each other and downstream targets, however, less is known about the functional roles of developmental important factors in other species due to lack of efficient loss-of-function models.

In this study a functional siRNA mediated knockdown model was established utilizing bovine COCs. A previous study in the Yao lab showed that microinjection specific KPNA7 siRNA in zygote stage of bovine embryo result in partial developmental arrest. Here this model was modified by microinjection of KPNA7 siRNA directly into COCenclosed oocytes and the injected oocytes were cultured in the presence of S-roscovitine for two days which proved to block the resumption of meiosis in $95 \%$ of GV oocyte (Coy, et al. 2005, Lee, et al. 2014). This additional incubation granted more time for targeting transcripts and reduction of translation therefore allowed a better siRNA knockdown effect. Cumulus cell layers is kept after microinjection in order to mimic the normal condition of conventional IVM procedure, where it is well established that maintenance of this layer is critical for successful IVF and embryo development.

At the end of oocyte growth, two types of maturation occur, cytoplasmic and nuclear. Both are essential for the formation of a competent oocyte for successful fertilization and development healthy embryos (Ajduk, et al. 2008, Rodriguez and Farin 2003). During 
nuclear maturation, meiotic arrest is released from prophase I and resumption of meiosis drives the progression to metaphase II (Eppig 1996). Of the known oocyte-specific genes, some encode proteins preferentially localized in the oocyte nucleus and are essential for oocyte maturation and subsequent embryo development. These oocyte-specific nuclear factors include Figla (Joshi, et al. 2007), Sohlh1/Sohlh2 (Choi, et al. 2008), Nobox (Rajkovic 2004), Ybx2 (Hammoud, et al. 2009) and NPM2 (Burns 2003, Lingenfelter, et al. 2011). The mechanism by which these oocyte-specific nuclear factors are transported into the nucleus is currently unknown. Our results showed that oocyte-specific KPNA7 is required for nuclear maturation of oocyte. As a nuclear protein transporter, KPNA7 has shown its capacity to interact and translocate oocyte-specific protein into nuclear in different species (Lingenfelter, et al. 2011) and our preliminary result also indicate bovine Nobox primarily interact with KPNA7 through three functional NLSs (data not shown). Therefore, the reduced maturation rate in KPNA7 knockdown oocytes might be due to the lack of nuclear transportation of the above-mentioned important factors recognized by KPNA7. However, further studies need to be done to look at the physical interaction of those factors and KPNA7.

Although KPNA7 has been found to interact with some of important oocyte specific factors, the interaction between functional factors forms a complicated network to control oocyte and early embryonic development. To further investigate factors that are direct or indirectly controlled by the KPNA7 network, a platform for RNA-seq of bovine 8-stage embryos after knockdown with KPNA7 was developed. Genome-wide transcriptome analysis revealed unique molecular gene regulation networks, which were related to 
macromolecule complex (GO:0032991) and subcellular localization (GO:0051179). Regulated transport of nuclear proteins into the nucleus through the nuclear pores is a vital highly regulated event in cells. The KPNA7 is one of the major cargo carriers from the cytoplasm into the nucleus. Macromolecules larger than $40 \mathrm{kDa}$ that cannot passively diffuse through the nuclear pores use a signal-mediated transport system (Lange, et al. 2007). Preliminary sequencing data showed a great potential to identify the functional network affected by KPNA7 knockdown. However, further experiments still need to be done to validate the differentially expressed genes and more analysis is needed to reveal the inner relationship between the differentially expressed genes.

\section{Conclusion}

In conclusion, the present studies strongly support a functional role of oocyte-specific KPNA7 in meiotic maturation of oocyte and a further role in promoting embryonic development to the blastocyst stage. A large number of genes that is affected by KPNA7 deficiency were documented, which enriched the depth of the functional study of KPNA7 network. Future studies are still need to further analyze the sequencing results in order to reveal more information on the role of KPNA7 in controlling the maternal effect gene expression network during early embryonic development in cattle. 


\section{Reference}

Ajduk, A, A Małagocki, and M Maleszewski 2008 Cytoplasmic maturation of mammalian oocytes: development of a mechanism responsible for sperminduced Ca2+ oscillations. Reproductive biology 8 3-22.

Andrews, S 2012 FastQC. A quality control tool for high throughput sequence data.. [h ttp://www. bioinformatics. bbsrc. ac. uk/projects/fastqc/].

Bettegowda, A, KB Lee, and GW Smith 2008 Cytoplasmic and nuclear determinants of the maternal-to-embryonic transition. Reprod Fertil Dev $\mathbf{2 0}$ 45-53.

Betthauser, JM, M Pfister-Genskow, H Xu, PJ Golueke, JC Lacson, RW Koppang, C Myers, B Liu, I Hoeschele, KJ Eilertsen, and GH Leno 2006 Nucleoplasmin facilitates reprogramming and in vivo development of bovine nuclear transfer embryos. Mol Reprod Dev 73 977-986.

Burns, K 2003 Roles of NPM2 in Chromatin and Nucleolar Organization in Oocytes and Embryos. Science (New York, NY) 300 633-636.

Choi, Y, D Yuan, and A Rajkovic 2008 Germ cell-specific transcriptional regulator sohlh2 is essential for early mouse folliculogenesis and oocyte-specific gene expression. Biology of reproduction 79 1176-1182.

Coy, P, R Romar, RR Payton, L McCann, AM Saxton, and JL Edwards 2005 Maintenance of meiotic arrest in bovine oocytes using the S-enantiomer of roscovitine: effects on maturation, fertilization and subsequent embryo development in vitro. Reproduction 129 19-26.

Diskin, M, M Parr, and D Morris 2011 Embryo death in cattle: an update. Reproduction, Fertility and Development 24 244-251.

Driver, AM, F Peñagaricano, W Huang, KR Ahmad, KS Hackbart, MC Wiltbank, and $\mathbf{H}$ Khatib 2012 RNA-Seq analysis uncovers transcriptomic variations between morphologically similar in vivo- and in vitro-derived bovine blastocysts. BMC Genomics 13118.

Eppig, JJ 1996 Coordination of nuclear and cytoplasmic oocyte maturation in eutherian mammals. Reproduction, Fertility and Development 8 485-489.

Geles, KG, and SA Adam 2001 Germline and developmental roles of the nuclear transport factor importin alpha3 in C. elegans. Development 128 1817-1830.

Hammoud, S, BR Emery, D Dunn, RB Weiss, and DT Carrell 2009 Sequence alterations in the YBX2 gene are associated with male factor infertility. Fertility and Sterility 91 1090-1095.

Hogarth, CA, S Calanni, DA Jans, and KL Loveland 2006 Importin alpha mRNAs have distinct expression profiles during spermatogenesis. Dev Dyn 235 253262.

Hu, J, F Wang, Y Yuan, X Zhu, Y Wang, Y Zhang, Z Kou, S Wang, and S Gao 2010 Novel importin-alpha family member Kpna7 is required for normal fertility and fecundity in the mouse. Journal of Biological Chemistry 285 3311333122 . 
Joshi, S, H Davies, LP Sims, SE Levy, and J Dean 2007 Ovarian gene expression in the absence of FIGLA, an oocyte-specific transcription factor. BMC Developmental Biology 767.

Lange, A, RE Mills, CJ Lange, M Stewart, SE Devine, and AH Corbett 2007 Classical nuclear localization signals: definition, function, and interaction with importin alpha. J Biol Chem 282 5101-5105.

Langmead, B, and SL Salzberg 2012 Fast gapped-read alignment with Bowtie 2. Nature methods 9 357-359.

Lee, K-B, G Wee, K Zhang, JK Folger, JG Knott, and GW Smith 2014 Functional Role of the Bovine Oocyte-Specific Protein JY-1 in Meiotic Maturation, Cumulus Expansion, and Subsequent Embryonic Development. Biology of reproduction.

Lingenfelter, BM, SK Tripurani, J Tejomurtula, GW Smith, and J Yao 2011 Molecular cloning and expression of bovine nucleoplasmin 2 (NPM2): a maternal effect gene regulated by miR-181a. Reprod Biol Endocrinol 940.

Mathe, E, H Bates, H Huikeshoven, P Deak, DM Glover, and S Cotterill 2000 Importin-alpha3 is required at multiple stages of Drosophila development and has a role in the completion of oogenesis. Dev Biol 223 307-322.

Ozawa, M, M Sakatani, J Yao, S Shanker, F Yu, R Yamashita, S Wakabayashi, K Nakai, KB Dobbs, MJ Sudano, WG Farmerie, and PJ Hansen 2012 Global gene expression of the inner cell mass and trophectoderm of the bovine blastocyst. BMC Developmental Biology 12 1-1.

Rajkovic, A 2004 NOBOX Deficiency Disrupts Early Folliculogenesis and OocyteSpecific Gene Expression. Science 305 1157-1159.

Rodriguez, K, and C Farin 2004 Developmental capacity of bovine cumulus oocyte complexes after transcriptional inhibition of germinal vesicle breakdown. Theriogenology 61 1499-1511.

Rodriguez, KF, and CE Farin 2003 Gene transcription and regulation of oocyte maturation. Reproduction, Fertility and Development 16 55-67.

Ruddock-D'Cruz, NT, VJ Hall, RT Tecirlioglu, and AJ French 2007 Gene expression analysis of single preimplantation bovine embryos and the consequence for developmental potential. Soc Reprod Fertil Suppl 64 341363.

Tejomurtula, J, K Lee, SK Tripurani, GW Smith, and J Yao 2009 Role of Importin Alpha8, a New Member of the Importin Alpha Family of Nuclear Transport Proteins, in Early Embryonic Development in Cattle. Biology of reproduction 81 333-342.

Trapnell, C, L Pachter, and SL Salzberg 2009 TopHat: discovering splice junctions with RNA-Seq. Bioinformatics 25 1105-1111.

Wang, X, KE Park, S Koser, S Liu, L Magnani, and RA Cabot 2011 KPNA7, an oocyte-and embryo-specific karyopherin $\alpha$ subtype, is required for porcine embryo development. Reproduction, Fertility and Development.

Wang, Z, M Gerstein, and M Snyder 2009 RNA-Seq: a revolutionary tool for transcriptomics. Nature reviews Genetics 10 57-63.

Xie, D, C Chen, L Ptaszek, S Xiao, X Cao, F Fang, H Ng, H Lewin, C Cowan, and S Zhong 2010 Rewirable gene regulatory networks in the preimplantation 
embryonic development of three mammalian species. Genome research $\mathbf{2 0}$ 804-815.

Yasuhara, N, N Shibazaki, S Tanaka, M Nagai, Y Kamikawa, S Oe, M Asally, Y Kamachi, H Kondoh, and Y Yoneda 2007 Triggering neural differentiation of ES cells by subtype switching of importin-alpha. Nat Cell Biol 9 72-79.

Yasuhara, N, R Yamagishi, Y Arai, R Mehmood, C Kimoto, T Fujita, K Touma, A Kaneko, Y Kamikawa, and T Moriyama 2013 Importin Alpha Subtypes Determine Differential Transcription Factor Localization in Embryonic Stem Cells Maintenance. Developmental cell 26 123-135.

Young, JC, AT Major, Y Miyamoto, KL Loveland, and DA Jans 2011 Distinct effects of importin $\alpha 2$ and $\alpha 4$ on Oct3/4 localization and expression in mouse embryonic stem cells. The FASEB Journal 25 3958-3965.

Zheng, P, and J Dean 2007 Oocyte-specific genes affect folliculogenesis, fertilization, and early development. Seminars in Reproductive Medicine $\mathbf{2 5}$ 243-251. 
Table 1. Summary of sequencing reads alignment to the reference genome

\begin{tabular}{llllll}
\hline Groups & Replicates & Reads & Clean reads & $\begin{array}{l}\text { Uniquely } \\
\text { mapped reads }\end{array}$ & $\begin{array}{l}\text { Uniquely } \\
\text { mapping ratio(\%) }\end{array}$ \\
\hline \multirow{3}{*}{ Control } & N1 & $28,348,792$ & $25,880,813$ & $20,620,620$ & 79.7 \\
& N3 & $37,232,404$ & $33,840,677$ & $28,756,764$ & 85.0 \\
& N4 & $32,258,893$ & $29,026,556$ & $23,279,108$ & 80.2 \\
& K1 & $31,927,469$ & 29.213 .213 & $23,588,476$ & 80.7 \\
\multirow{2}{*}{ Knock out } & K2 & $27,018,092$ & $25,335,988$ & $19,088,018$ & 75.3 \\
& K3 & $37,332,467$ & $35,666,342$ & $31,388,002$ & 88.0 \\
Total & & $194,118,117$ & $178,963,589$ & & \\
\hline
\end{tabular}




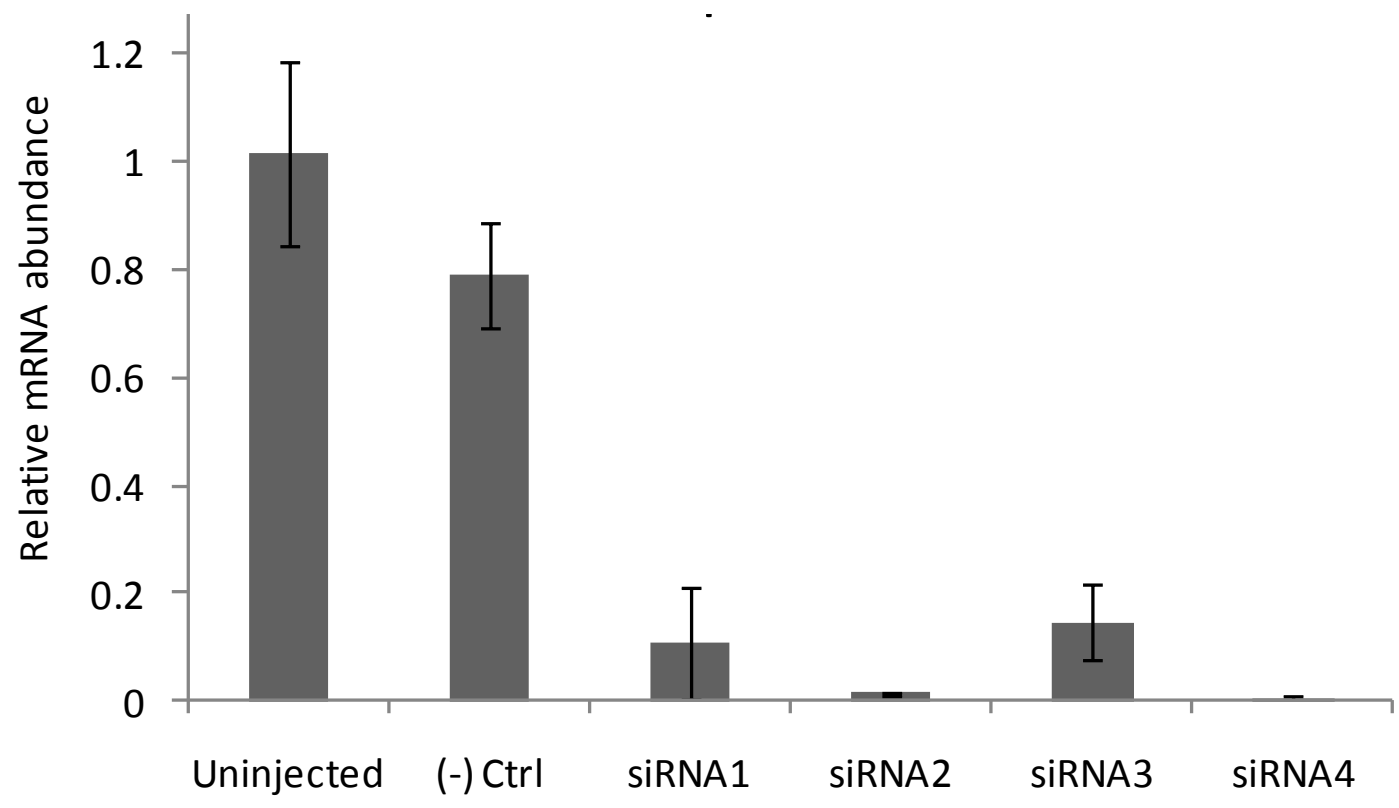

Figure 1. Validaton of siRNAs 4 siRNA species were microinjected into bovine COCs and control group were injected with sham siRNA. Effect of knockdown KPNA7 is evaluated using real-time PCR. 


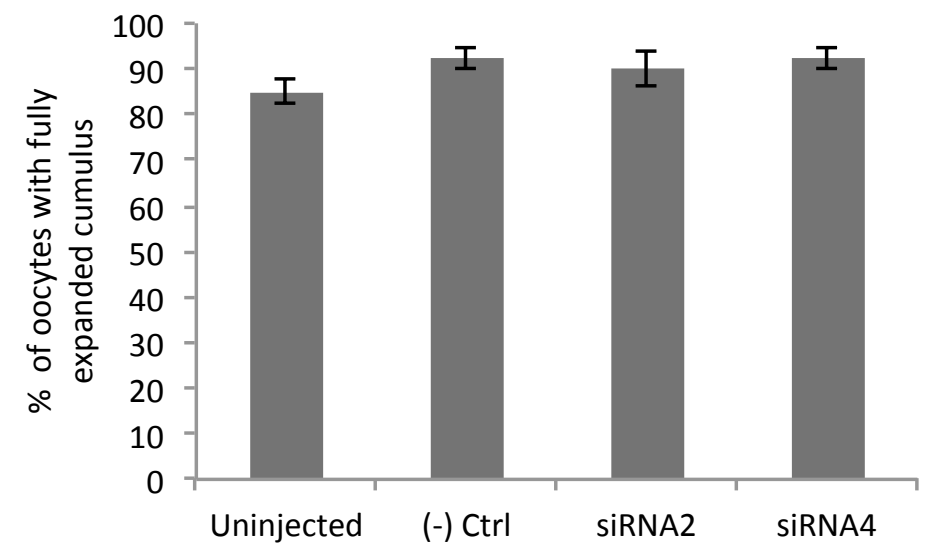

Figure 2a. Effect of microinjection of KPNA7 siRNA into cumulus-enclosed bovine germinal vesicle stage oocytes on expansion of the cumulus cell layer during in vitro maturation. Knockdown of KPNA7 does not affect cumulus cell expansion. Degree of cumulus expansion were determined at $72 \mathrm{~h}$ post injection. 


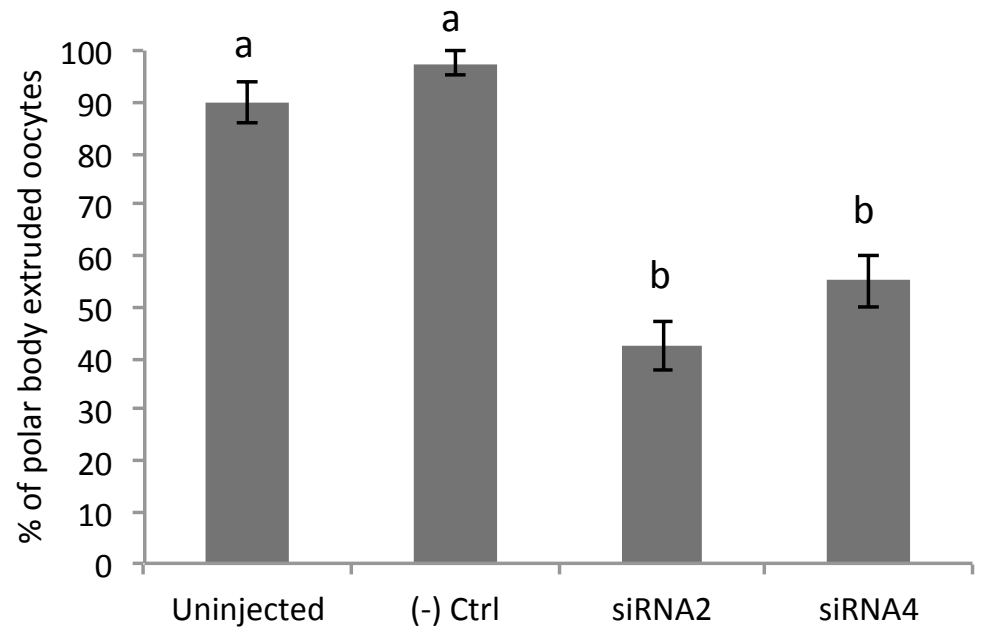

Figure 2b. Knockdown of KPNA7 significantly reduced the oocyte maturation rate. Proportion of oocytes progressing to metaphase II were determined by the extrusion of the $1^{\text {st }}$ polar body. 


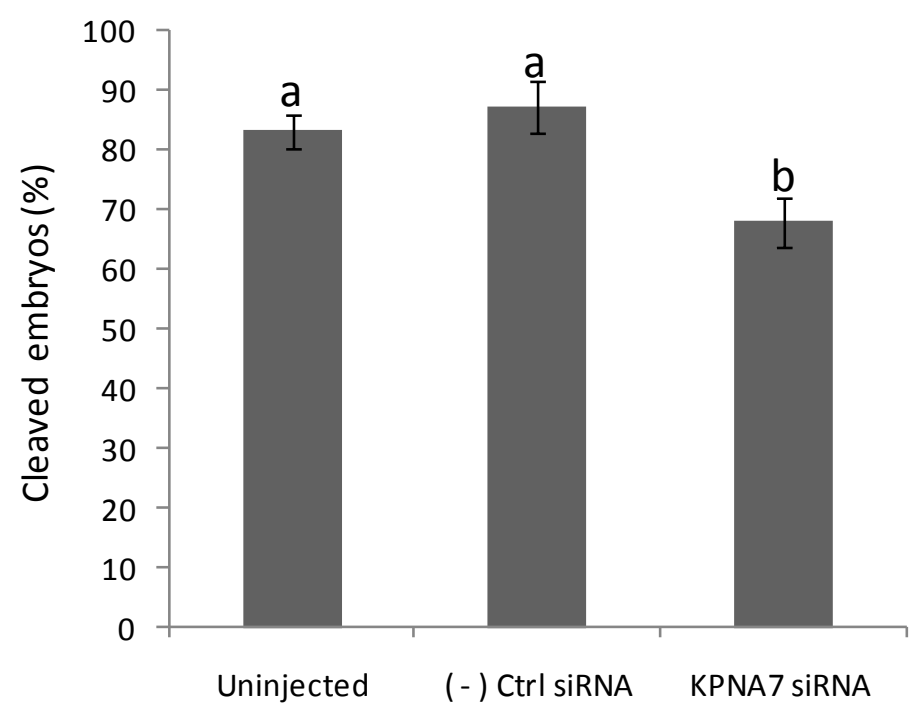

Figure 3a Effect of KPNA7 knockdown on first cleavage of embryos following in vitro fertilization. KPNA7 knockdown result in a significant reduction of cleaved embryos observed $48 \mathrm{~h}$ post insemination. 


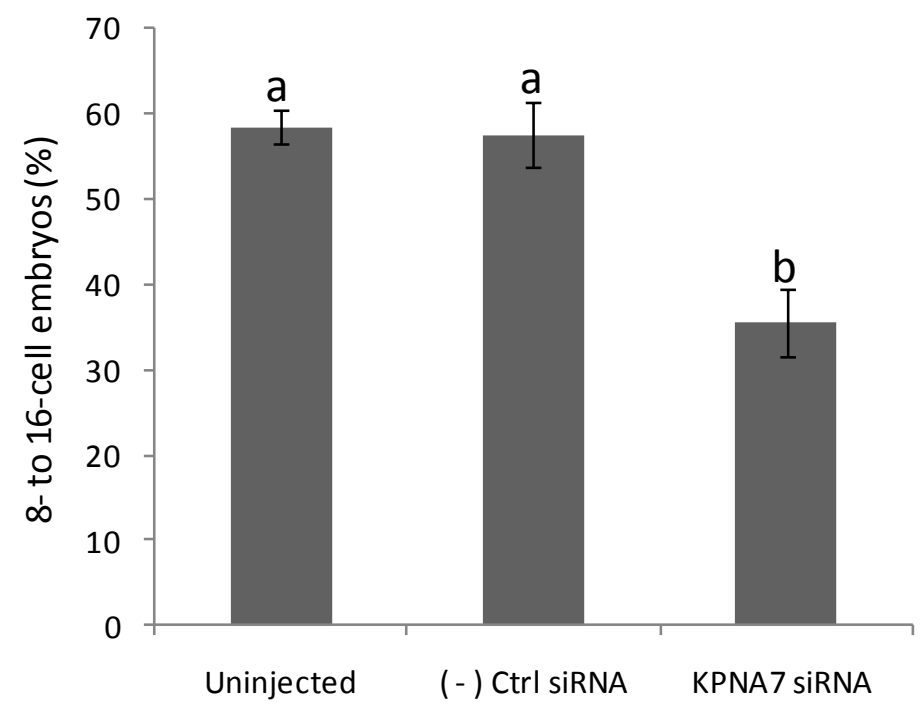

Figure 3b. KPNA7 knockdown result in a significant drop in early embryonic stage from 8 to 16 cell stages, which is the critical time of maternal to zygotic transition in bovine embryogenesis. 


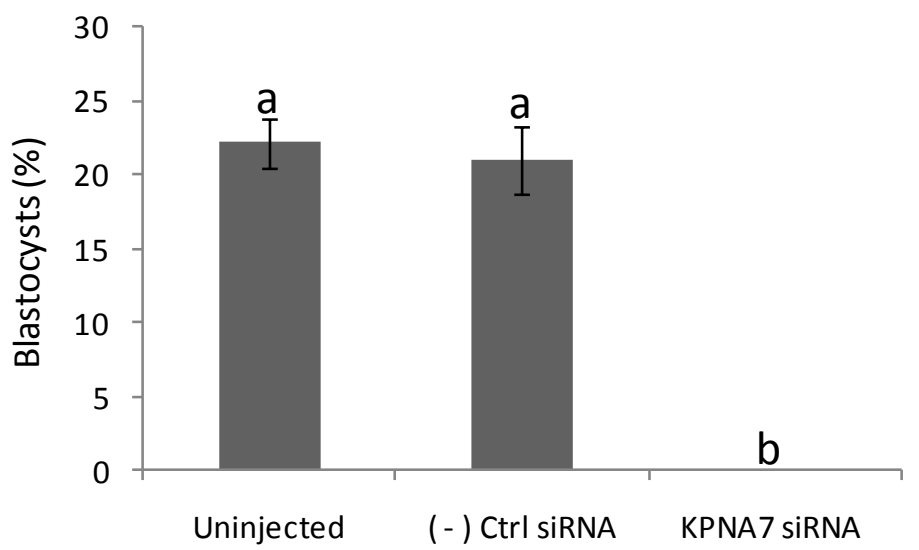

Figure 3c. Effect of microinjection of KPNA7 siRNA into cumulus-enclosed bovine germinal vesicle stage oocytes on blastocyst development following IVF. KPNA7 siRNA microinjection result in no blastocyst embryos. 


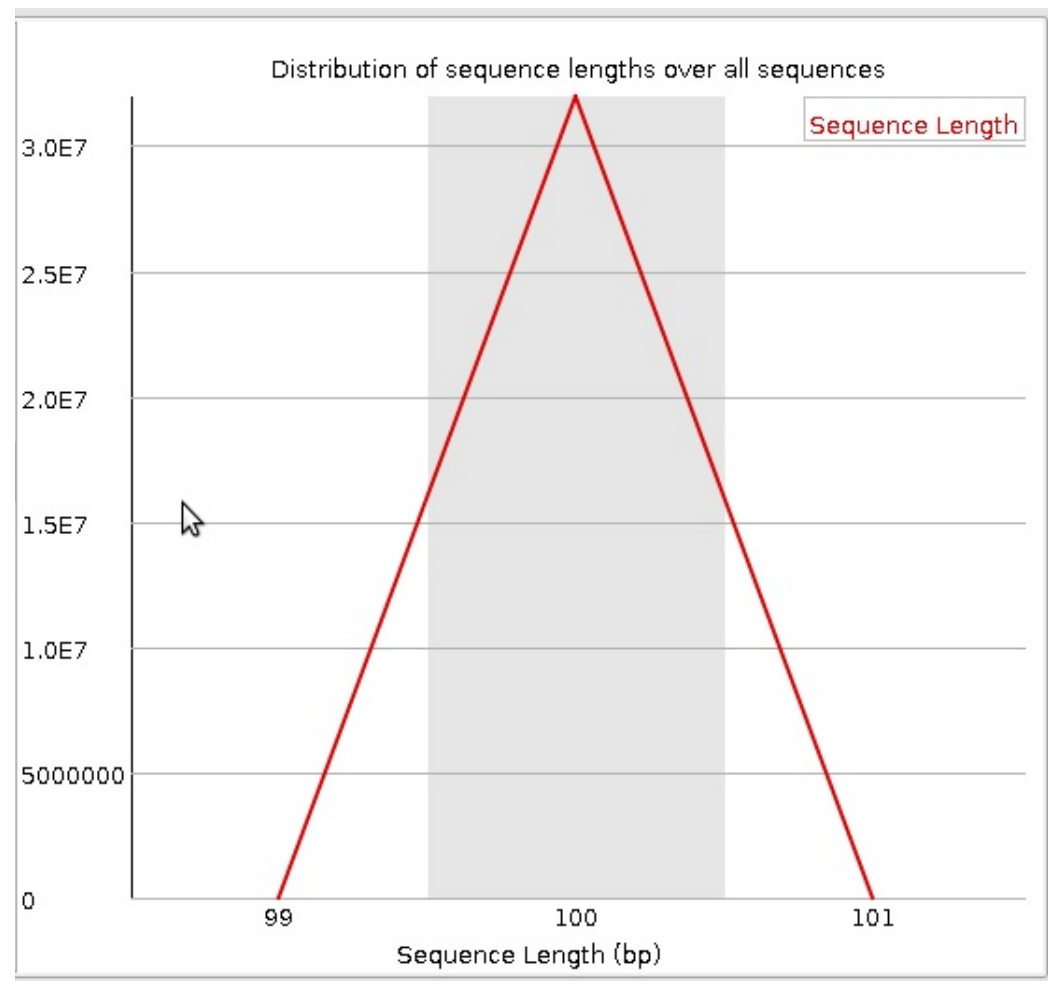

Figure 4. Summary of reads length range. The reads length range from 99 to 101 with a peak length at $100 \mathrm{bp}$. 


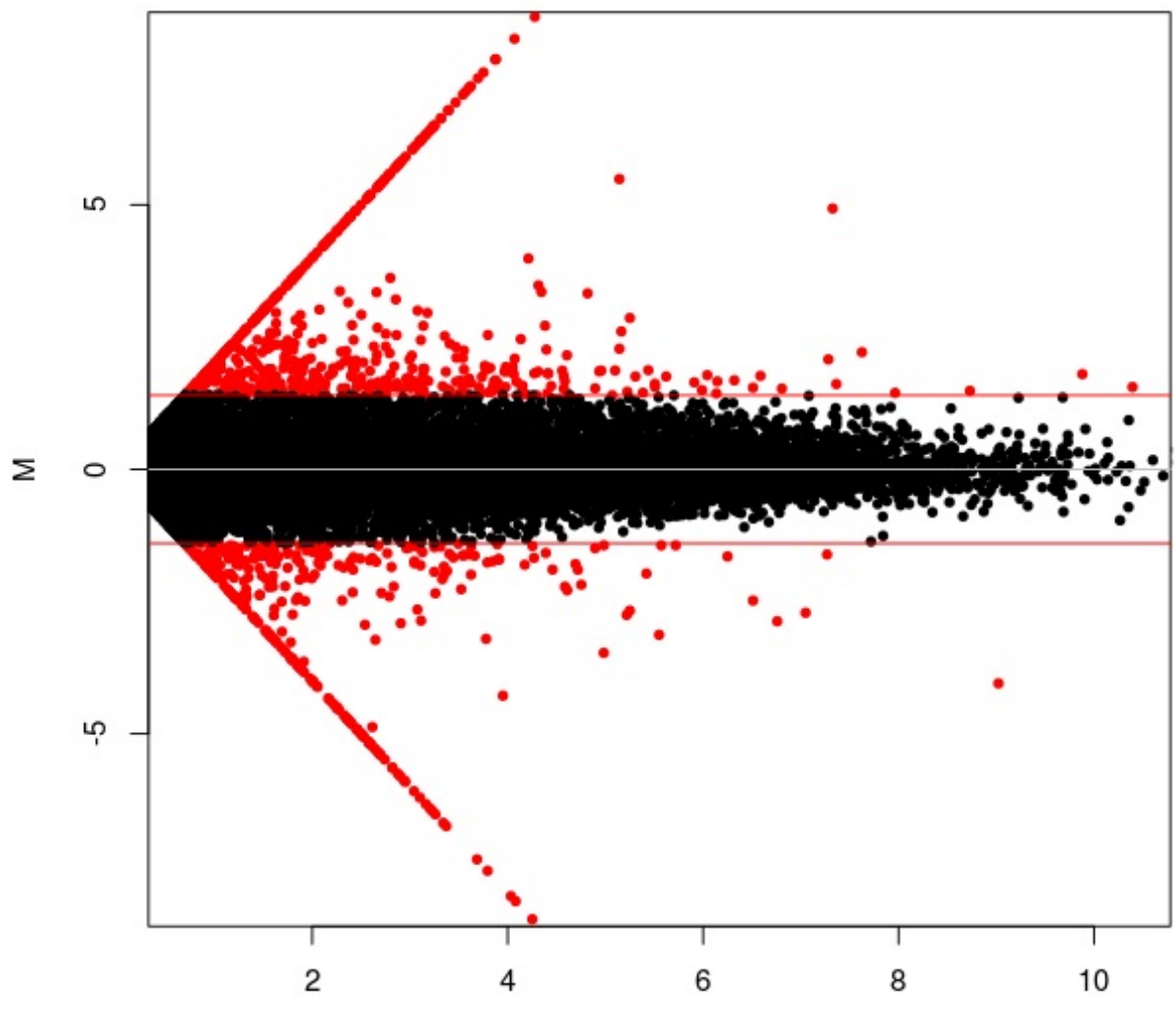

A

Figure 5. M-A plot was generated to display the $\log 2$ fold change (the $\log 2$ ration of expression levels for each gene between the two experimental groups) against the average $\log 2$ expression (the overall average expression level for each gene across the two experimental groups). Most of the genes showed a fold change close to 0 (black dots), and differentially expressed genes were shown in red dots 


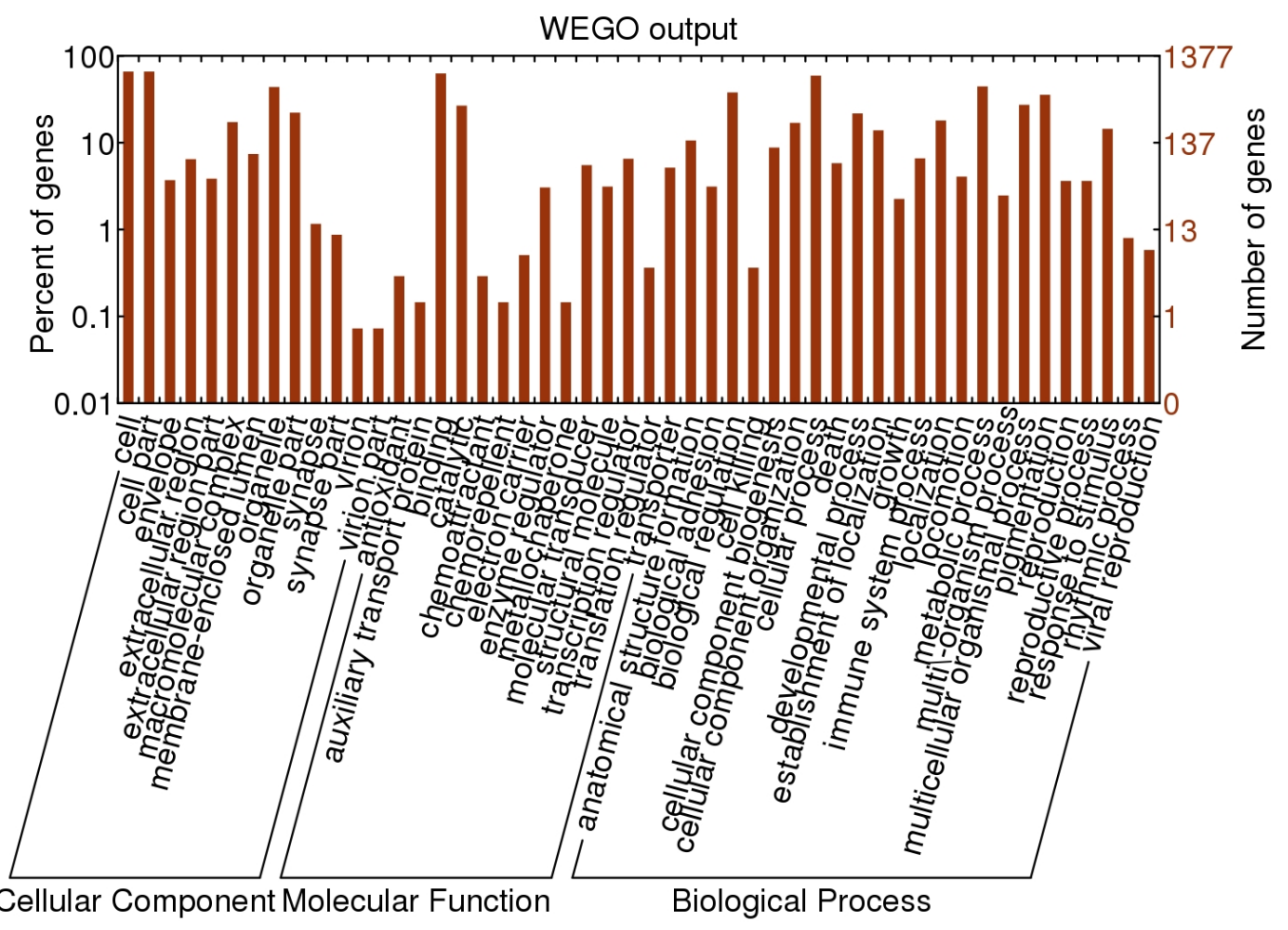

Figure 6. Comparative analysis and functional classification of gene ontology analysis of differential expressed gene between KPNA7 injected and non-injected embryos. 


\section{Appendix}

\section{Part I Preliminary Studies}

\section{Bovine KPNA7 interact with Nobox but not Oct 4 revealed by co- immunoprecipitation using HeLa cells}

\section{Introduction}

Nuclear transcription factors are required for initiation of transcription in early embryos before embryonic genome activation. The transportation of transcription factor is mediated by karyopherins (KPNs), which facilitate the translocation of proteins containing nuclear localization signals (NLSs) from the cytoplasm into the nucleus. To date, seven KPNA proteins (KPNA1 to 7) have been characterized in mammals, of which KPNA7 is specifically expressed in oocytes and early embryos. Recent studies have shown that KPNA7 is an important maternal factor required for early embryonic development and KPNA7 knockout mice display abnormal gene expression and epigenetic modifications. Furthermore, the nuclear transportation function of KPNAs protein family also led us to hypothesize that the cargo proteins for KPNA7 are important at specific stages of oocyte maturation and embryonic development. NOBOX (newborn ovary homeobox-encoding gene) and Oct4 are key transcription factor that are preferentially expressed in oocytes and are essential for folliculogenesis and early embryonic development. Lack of NOBOX and Oct4 disrupts expression of many germcell specific genes. Using HeLa cells as a model system, we found that KPNA7 protein interacts with NOBOX with highest intensity compare with other KPNAs via coimmunoprecipitation. Using NLSmapper, a program for prediction of NLS specific to the KPNA pathway, putative NLSs were detected on NOBOX. Domain deletion studies 
further revealed the classic monopartitte NLS near the C-terminus of NOBOX is required for their interactions. However, Oct4 showed interaction with KPNA2 but not KPNA7 which is consistent with the results in previous human study about Oct4. This study will enable further elucidation of the molecular mechanisms involved in KPNA7 oocyte specific pathway and might shed further light on key regulators during maternal embryonic transition.

\section{Materials and Methods}

\section{Construction of Expression Vectors}

Bovine KPNA7 ORF and Nobox ORF with various mutations were cloned into mammalian expression vector pcDNA3.1a with Flag tag and myc tag N-terminal (Noboxflag and KPNA7-myc; Table 1).

\section{Co-Immunoprecipitation}

HeLa cells were grown in DMEM supplemented with $10 \%$ FBS. Transfections were performed using X-tremegene 9 transfection reagent (Roche). Cells were cultured for 24 or 48 hours after transfection and co-immunoprecipitation was performed using Pierce co-immunoprecipitation kit. Western blot were performed followed the protocol described in Chapter 1.

\section{Results and Discussions}

\section{KPNA7 interacts with Nobox (Figure 1.)}

A strong interaction between KPNA7 and NOBOX were detected (Figure 1). The flow through was used as control to verify the protein expression and binding efficiency 
Newborn ovary homeobox gene (NOBOX) is a transcription factor that is preferentially expressed in oocytes and essential for folliculogenesis. NOBOX knockout mice are infertile and lack of NOBOX disrupts expression of many germ-cell specific genes and microRNAs. In bovine, NOBOX gene knockdown studies indicate it is a maternal effect gene that is essential for early embryonic development. This interaction shows the involvement of KPNA7 in the NOBOX functional pathway and also indicates the potential role KPNA7 might play in mediating other important factors.

\section{Site directed mutations and domain deletions of NOBOX NLS disrupts the interaction with KPNA7 (Figure 2a and b)}

Although the interactions were still detectable in all the mutations on multiple NLS, the signals were weaker compare to the wild type NOBOX. After delete the first NLSs, the interaction is completely disrupted. As NLS signals on the cargo proteins are critical for recognition by KPNA proteins, further studies of the interaction between KPNA7 and mutated NOBOX NLSs is need. Using NLSmapper, a program designed to predict NLS sequence, three putative NLSs (start with amino acid residues 42, 143 and 168) were detected on the NOBOX protein. Site directed mutations of the NLSs were made on the critical arginine and lysine residues on NOBOX protein. Although the interactions were still detectable in all mutations on multiple NLS, the signals were weaker compared to the wild type NOBOX. As NOBOX contains two bipartite NLSs, which is separated by around 10 residues, deletion of the entire NLS domain may show dramatic effects on the interaction with KPNA7. The domain deletion co-ip showed the classic bipartite NLS near the $\mathrm{C}$-terminus of NOBOX is required for the interaction. Furthermore, this bipartite 
NLS also has the highest score in the NLSmapper prediction, which suggests a higher NLS activity.

\section{The differentrial interaction pattern between KPNA7 and developmentally important genes NOBOX and Pou5F1 (Figure 3 a and b.)}

The interaction between KPNA7 and NOBOX is higher than with other KPNAs except with KPNA2. On the other hand the result showed Oct4 interacts with KPNA2 but not KPNA7. As sharing high a degree of identity, it is reasonable to see that KPNA7 and KPNA2 might interact with the same protein at similar intensity. However, their functions are quite different, as differential interaction has been observed with other partner proteins, such as Oct4. The result showed Oct4 interacts with KPNA2 but not KPNA7, which is consistent with the results in previous human study.

\section{Conclusion}

We used co-immunoprecipitation experiments to evaluate the interaction of KPNA7 with oocyte-specific nuclear factors (e.g. Oct4 and Nobox). Our preliminary data indicate that KPNA7 interacts with Nobox, an oocyte-specific transcription factor important for folliculogenesis and early embryonic development. This finding help us to reasure the ability of KPNA7 to bind with oocyte-specific nuclear factors critical to development 
Table 1. Constructed Expression Vectors

\begin{tabular}{|c|c|c|c|c|}
\hline Insert & Vector & Tag & Plasmid & Experiment \\
\hline Kpna7 ORF & pcDNA3.1a & Kozak / cmyc & KPNA7ORFpcDNA & Co-IP \\
\hline Nobox ORF & pcDNA3.1a & Kozak / Flag & NoboxORFpcDNA & Co-IP \\
\hline Nobox mutation on K118A, K119A & pcDNA3.1a & Kozak / Flag & mutK118A/K119A & Site Mutation Co-IP \\
\hline Nobox mutation on K146A, R147A & pcDNA3.1a & Kozak / Flag & mutK146A/R147A & Site Mutation Co-IP \\
\hline Nobox mutation on R168A, R169A & pcDNA3.1a & Kozak / Flag & mutR168/R169A & Site Mutation Co-IP \\
\hline Nobox Deletion from 231bp & pcDNA3.1a & Kozak / Flag & Nobox $\Delta 231$ pcDNA & Domain deletion Co-IP \\
\hline Nobox Deletion from 372bp & pcDNA3.1a & Kozak / Flag & Nobox $\triangle 372$ pcDNA & Domain deletion Co-IP \\
\hline Nobox Deletion from 588bp & pcDNA3.1a & Kozak / Flag & Nobox $\Delta 588$ pcDNA & Domain deletion Co-IP \\
\hline Oct4 ORF & pcDNA3.1a & Kozak / Flag & Oct4ORFpcDNA & Co-IP \\
\hline
\end{tabular}




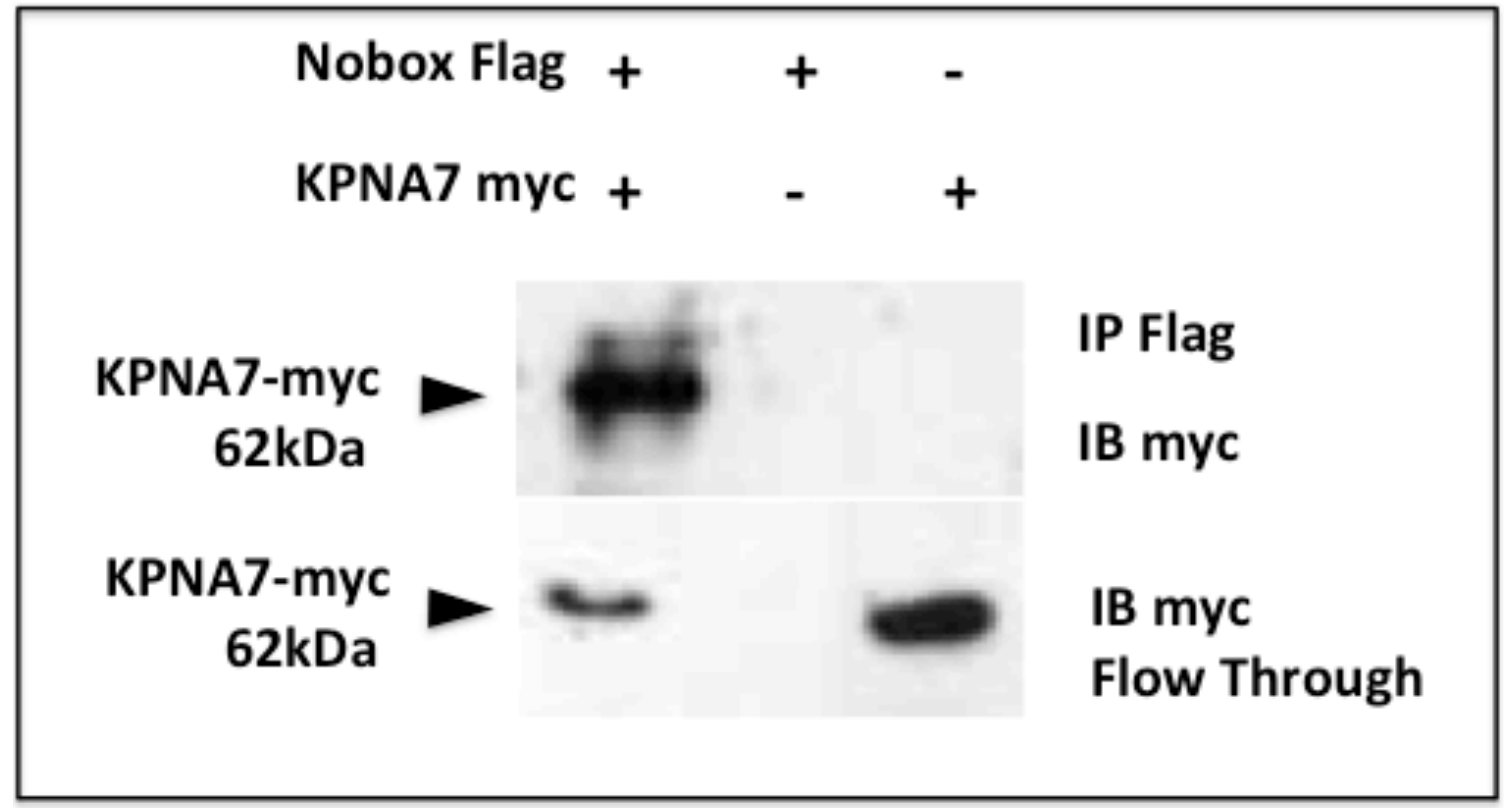

Figure 1. bovine KPNA7 interacts with full length Nobox 


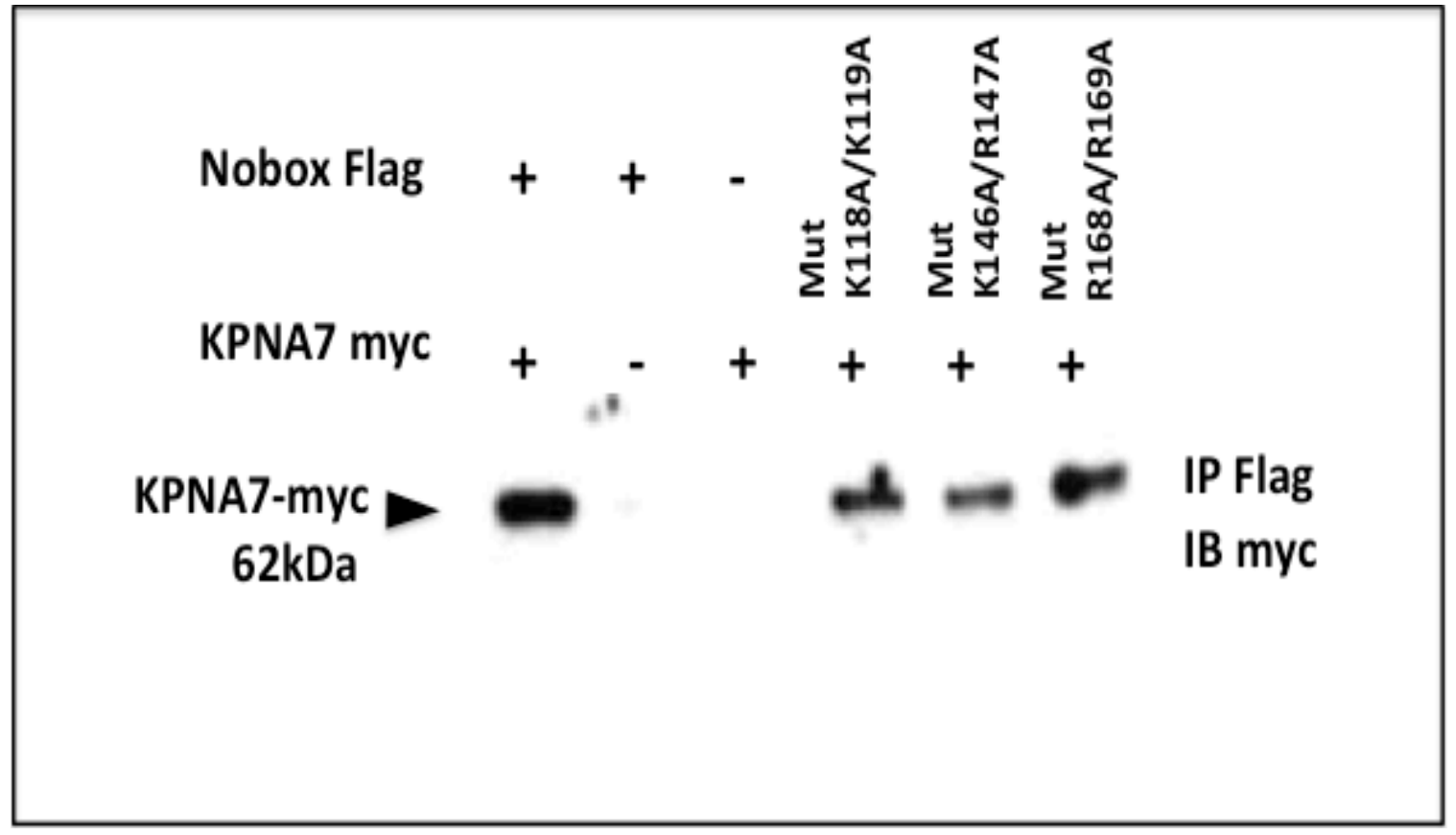

Figure 2a. bovine KPNA7 interact with Nobox through multiple NLSs 


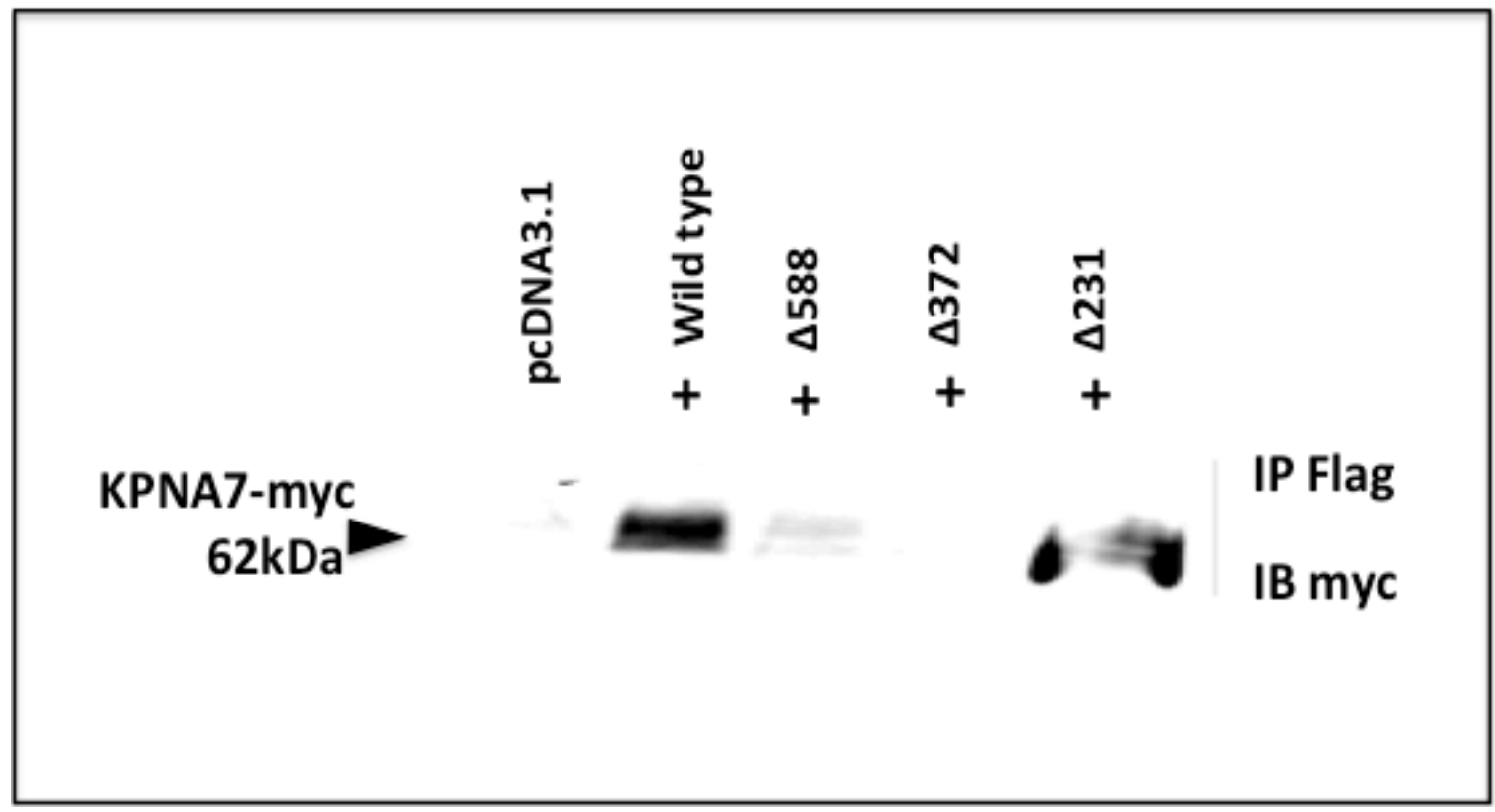

Figure 2b. bovine KPNA7 interact with Nobox through multiple NLSs 


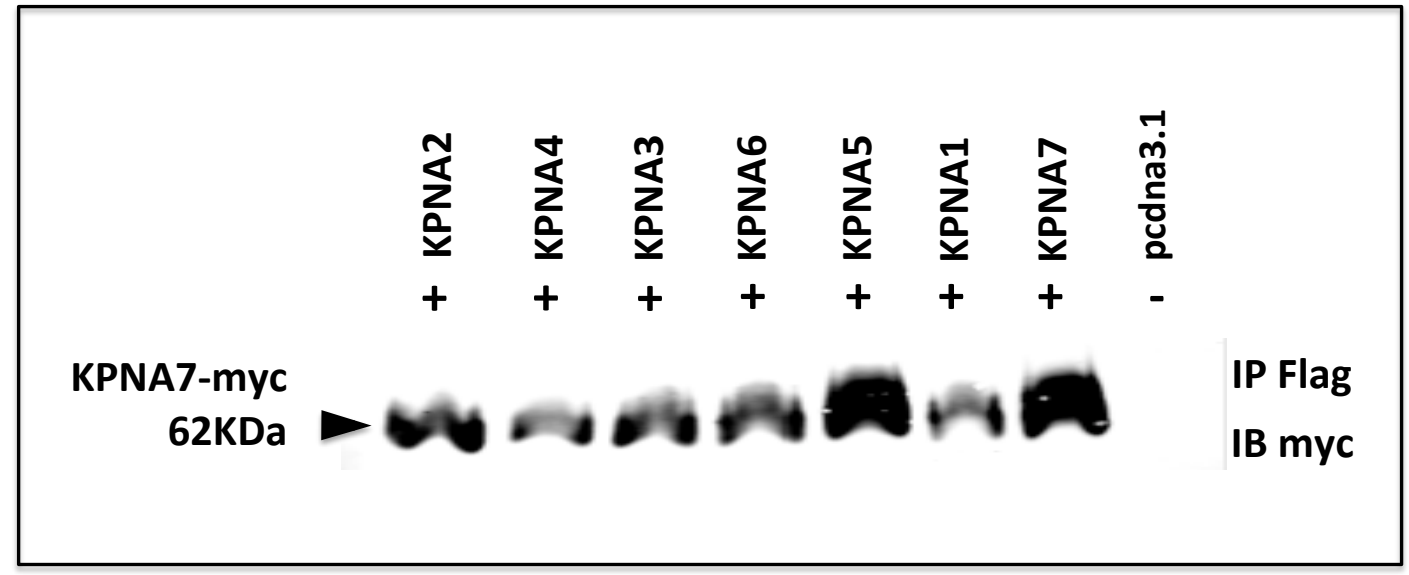

Figure 3a Bovine KPNA7 in the primary interaction partner of Nobox 


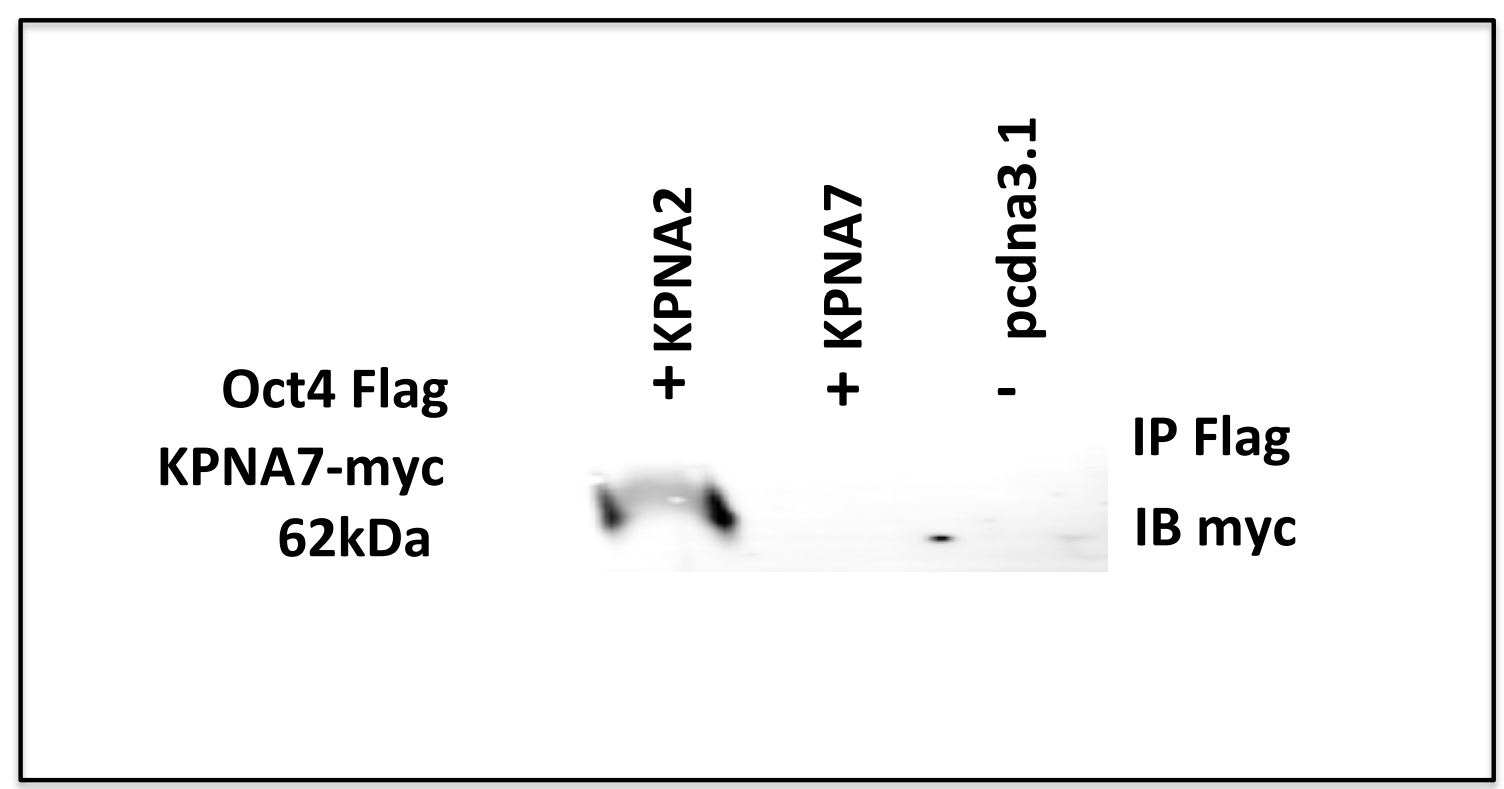

Figure $3 b$. Bovine Oct4 interact with KPNA2 instead of KPNA7 


\section{Appendix}

\section{Part II Protocols}

\section{Protocol I: Fixation of tissues}

\section{Fixation of tissue for in situ hybridization}

$1 \mathrm{Cut}$ tissue into $2 \mathrm{~mm}$ thick pieces

2 Incubate cut tissue at $4{ }^{\circ} \mathrm{C}$ for $24 \mathrm{~h}$ at freshly made $4 \%$ paraformaldehyde $(\mathrm{pH}=7.5)$

3 Wash the tissue sample with PBS 4 times/15min at room temperature.

4 Store them in methanol at $-20^{\circ} \mathrm{C}$ until use.

$4 \%$ paraformaldehyde $(500 \mathrm{ml})$

1 paraformaldehyde $20 \mathrm{~g}$

$2 \mathrm{dd} \mathrm{H} 2 \mathrm{O} \quad 332 \mathrm{ml}$

3 dissolve at $60^{\circ} \mathrm{C}$ with stirring (it might take long time to dissolve)

4 add 1 drop of $2 \mathrm{~N} \mathrm{NaOH}$ to make the solution clear

5 add $168 \mathrm{ml}$ of $3 \mathrm{X}$ PBS

6 adjust $\mathrm{pH}$ to 7.2 by $\mathrm{HCl}$

Store at $4{ }^{\circ} \mathrm{C}$ until use

use RNase free container to prepare the solution

\section{Fixation for Immunohistochemistry}

1 Cut tissue into $2 \mathrm{~mm}$ thick pieces

2 Incubate cut tissue at $4{ }^{\circ} \mathrm{C}$ for $24 \mathrm{~h}$ at freshly made Davidsm's solution

3 Transfer the tissue sample to $70 \%$ ethanol and store at $4{ }^{\circ} \mathrm{C}$

this can be store for 6 months

Davidsm's Solution

$137 \%$ formalin 2 parts

$2100 \%$ ethanol 3 parts

3 Glacial Acetic Acid 1 parts

4 Water 3 parts 


\section{Protocol II: Embedding Tissue in Paraffin}

\section{Method I(Longer time)}

Prepare Ethanol, Blue cap tubes, Water Container, Water bath Melted Paraffin, Moding block, Foil, Forceps

1 from the fixation solution, place the fixed tissue into 50\% Ethanol and incubate for $45 \mathrm{~min}$

2 Transfer the tissue into $70 \%$ Ethanol and incubate for $45 \mathrm{~min}$

3 Transfer the tissue into $95 \%$ Ethanol and incubate for $45 \mathrm{~min}$

4 Transfer the tissue into $100 \%$ Ethanol and incubate for $45 \mathrm{~min} \times 2$

5 Transfer the tissue to Xylen for and observe the transparent condition of the tissue

6 Transfer the tissue to Half Xylene and half paraffin for $2 \mathrm{~h}$

7 Transfer the tissue to paraffin $2 \mathrm{~h}$

8 Embedding the tissue

\section{Method II (Shorter time)}

Prepare Ethanol, Blue cap tubes, Water Container, Water bath Melted Paraffin, Moding block, Foil, Forceps

1 from the fixation solution, place the fixed tissue into

$50 \%$ Ethanol and incubate for $30 \mathrm{~min}$

$270 \%$ Ethanol and incubate for $30 \mathrm{~min}$

$395 \%$ Ethanol and incubate for 30min

$4100 \%$ Ethanol and incubate for 30min x 2

$5100 \%$ Ethanol and Xylene $(1: 1 \mathrm{v} / \mathrm{v})$ half and half solution incubate for $30 \mathrm{~min}$

6 Xylene Ethanol and incubate for $30 \mathrm{~min} \times 2$

7 Half Xylene and half paraffin incubate for 30min

8 Paraffin incubate for $1 \mathrm{~h} \times 2$

9 Embedding the tissue 


\section{Protocol III: Immunohistochemistry Protocol For Rainbow Trout Ovary}

From Pierce $\mathrm{ABC}$ peroxidase staining kit with Methal enhance DAB substrate kit 1. Prepare paraffin embedded tissue section slides dehydrate the slides through desending ethanol concentrations with

xylene $3 \times 5 \mathrm{~min}$,

$100 \%$ ethanol $3 \times 5 \mathrm{~min}$, $95 \%, 80 \%, 70 \%, 50 \% 5$ min each

2. Wash the paraffin embedded tissue section with ultrapure water for $5 \mathrm{~min}$

3. Endogenous peroxidase activity can be quenched by incubating tissue for $30 \mathrm{~min}$ in $0.3 \% \mathrm{H} 2 \mathrm{O} 2$ in Methanol

4. Wash slide for 20 min with PBS

5. Block the slide for 20min with the blocking buffer

Preparation of blocking buffer:

$10 \mathrm{ml} \quad$ PBS

3 drops Blocking Serum

$1 \%$ BSA (optional)

6. Blot excess blocker form the tissue sections. Apply the Primary Antibody and incubate tissue for $30 \mathrm{~min}$.

Preparation of Primary AB:

adjust to appropriate concentration with Blocking Buffer

7. Wash slide for 10 min with PBS. Apply the biotinylated Secondary Antibody and incubate tissue for $30 \mathrm{~min}$

Preparation of 2nd Biotinylated Secondary AB

$10 \mathrm{ml} \quad$ PBS

3 drops $(135 \mu l)$ Blocking Serum

1 drop $(45 \mu \mathrm{l}) \quad$ Biotinylated Secondary Antibody (from ultra sensitive ABC )

8 Note the ABC Reagent may be prepared during this incubation step

Preparation of $\mathrm{ABC}$ Reagent: 


\section{PREPARE REAGENT 30 MIN BEFORE USE!!!}

$10 \mathrm{ml} \quad$ PBS

4 drops $(180 \mu l) \quad$ Reagent A

4drops $(180 \mu l) \quad$ Reagent B

immediately mix solution and allow $30 \mathrm{~min}$ for the complex to form

9. Wash slides for 10 min PBS. Add the ABC Reagent to the tissue section and incubate for 30min

10. Wash slide for 10min with PBS. Remove the excess buffer.

11. Add the Metal inhanced DAB substrate working solution to the tissue section and incubate until the desire color show(5-15min)

Preparation of DAB working solution $5 \mathrm{ml}$

$450 \mu 1$ Stable Peroxide Buffer from $4^{\circ} \mathrm{C}$

$50 \mu 1$ DAB 10X solution from -20 return immediately (if precipitate do not use)

12. Dehydrate the slide with $50 \%, 70 \%, 95 \%, 100 \%$ ethanol clear with xylene (5min each) and cover with mounting solution with coverslip.

13. Wash slide for 5 min with PBS. Counterstain if desired

Trouble Shooting

- Note the time required to perform the above protocol may be reduced with a slight loss in sensitivity by performing the following alterations

- Shorten the incubation steps with the primary antibody the biotinylated secondary antibody and the $\mathrm{ABC}$ Reagent to 10 min each

- Rinse tissue with a gentle stream of ultrapure water instead of the $10 \mathrm{~min}$ wash after each incubation step

- Because the primary antibody is prepared in Blocking Buffer the incubation steps with the Blocking Buffer and the Primary Antibody mat be combined

- Increase the concentration of the Primary Antibody and decrease the incubation times to 2 min. Optimization of the concentrations may be required.

14. Counterstaining (Optional) 
After staining tissue with the PBC staining kit, a counterstain may be useful for improving the contrast between the background and the colored reaction products. Two main considerations in the choice of a counterstain are the color of the dye and the $\mathrm{pH}$ characteristics of the dye. An acidic stain produces an anion chromophore upon dissociation. Conversely, a dye is a basic stain if its chromophore is a positively charged ion. Basic days have an affinity for the relatively acidic components of tissue, such as DNA, RNA and the nucleus. Acidic Dyes preferentially stain the basic constituents of tissue, such as the cyplasm. 


\section{Protocol IV: Western Blot using Odyssey scanner}

\section{Materials}

Ready gel (Bio-Rad)

$1 \mathrm{X}$ running buffer

1X transfer buffer

$1 \mathrm{X}$ PBS $+0.1 \%$ Tween-20 (PBST)

PBS $+5 \%$ non fat milk (blocking buffer)

PBST $+5 \%$ non fat milk (for primary antibody)

PBST $+5 \%$ non fat milk with $0.2 \%$ Tween $20+0.01 \%$ SDS (for secondary antibody)

Protein samples: HeLa cells transfected with bovine KPNA7-Myc plasmid. Cells were lysed in lysis buffer followed by addition of 5X Loading buffer. 4 samples/group .

Rabbit anti-c-Myc antibody (primary antibody 1 )

Monoclonal anti-alpha-Tubulin antibody (primary antibody 2)

IRDye 800CW Goat Anti-Rabbit Secondary Antibody (Odyssey) - green

IRDye 680RD Goat Anti-Mouse Secondary Antibody (Odyssey) - red

\section{Electrophoresis}

1. Cut along the dotted line at the bottom of the ready gel cassette with a razor blade.

2. Pull the bottom tape off the ready gel cassette to expose the bottom of the gel.

3. Gently remove the comb and rinse the wells with $1 \mathrm{x}$ running buffer using a syringe.

4. Assemble the Mini-PROTEAN electrophoresis module and fill the tank with $1 \mathrm{X}$ running buffer.

5. Boil the protein samples for 5 min and spin briefly.

6. Load $10 \mu 1$ of pre-stained protein standards into the first well.

7. Load $15 \mu 1$ of each sample (4 samples) into the next 4 wells.

8. Run the gel at $100 \mathrm{~V}$ for 1 hour.

\section{Transfer (Western blotting)}

9. Cut a PVDF membrane and 6 filter papers to the size of the gel. 
10. Place the PVDF membrane in $100 \%$ methanol for 5 minutes.

11. Place the filter papers, the PVDF membrane and 2 fiber pads separately in $1 \mathrm{X}$ transfer buffer for 5 minutes.

12. Remove the gel carefully from the cassette and transfer to $1 \mathrm{x}$ transfer buffer and shake for 5 minutes.

13. Place the gel holding cassette, with the grey side down on a clean surface.

14. Place one pre-wet fiber pad on the gray side of the cassette.

15. Place 3 sheets of filter paper on the fiber pad.

16. Place the gel on the filter papers.

17. Place the pre-wet membrane on the gel.

18. Place another 3 sheets of filter paper on the membrane.

19. Roll the sandwich with a plastic pipette to get rid of air bubbles.

20. Place another pre-wet fiber pad on the filter papers.

21. Lock the cassette and insert the cassette into a transfer module.

22. Place the module, an ice block and a magnetic stirring bar in the tank filled with cold $1 \mathrm{X}$ transfer buffer.

23. Set the voltage to $100 \mathrm{~V}$ and run for 1 hour.

\section{Immunodetection}

24. Place the membrane in $100 \%$ methanol for $1 \mathrm{~min}$.

25. Rinse the membrane with water.

26. Place the membrane in $1 \mathrm{X}$ PBS for 2 min and leave the membrane in PBS until further steps (never let the membrane dry!).

27. Place the membrane in $5 \mathrm{ml}$ PBS blocking buffer for 1 hour at RT with gentle shaking.

28. Incubate with 1:500 diluted rabbit anti-myc antibody (primary antibody 1) and 1:1000 diluted monoclonal anti-alph-Tubulin-anti antibody (primary antibody 2) in $5 \mathrm{ml}$ of PBST milk buffer overnight at $4{ }^{\circ} \mathrm{C}$ with gentle shaking.

29. Pour off primary antibodies solution and rinse the membrane with $1 X$ PBST

30. Wash the membrane in $1 \mathrm{X}$ PBST for $5 \mathrm{~min}, 3$ times with shaking at RT. 
31. Pour off $1 \mathrm{X}$ PBST, add $5 \mathrm{ml}$ PBST $+5 \%$ non fat milk containing $0.2 \%$ Tween-20 and $0.01 \%$ SDS.

32. Dilute IRDye $800 \mathrm{CW}$ goat anti-rabbit secondary antibody 1: 10,000 times

33. Dilute IRDye 680RD goat anti-mouse secondary antibody 1: 10,000 times into the solution.

34. Wrap the box with aluminum foil and incubate at RT for another 15 minute with gentle shaking.

35. Wash the membranes with 1X PBST for 5 minute, 3 times with shaking at RT.

36. Rinse the membrane with $1 \mathrm{X}$ PBS, and the membrane can be scanned wet or dry. 


\section{Protocol V Preparation of Yeast Competent Cells \&Transformation of Yeast}

1 inoculate $3 \mathrm{ml}$ YPDA with $2-3 \mathrm{~mm}$ yeast 2 colonies one per tube. Grow overnight at $30^{\circ} \mathrm{C}$ with shaking $250 \mathrm{rpm}$ (check OD 600 0.2-0.3)

2 use 2 corvettes one is YPDA as the blank, measure OD at $600 \mathrm{~nm}$

3 Shaking $100 \mathrm{ml}$ YPDA in $30^{\circ} \mathrm{C}$ at 250rpm for 2-3hrs check OD600 0.4-0.6

4 Transfer cells in $50 \mathrm{ml}$ tube and centri at 5000rpm for $5 \mathrm{~min}$ at RT.

5 Decant the supernatant and resuspend cell in $1 \mathrm{ml}$ sterile water, transfer cell to $1.5 \mathrm{ml}$ tube spin down

6 resuspend in $0.5 \mathrm{ml} 1 \mathrm{xTE} / 1 \mathrm{x} \mathrm{LiOAc}$

made fresh from 10xTE $0.1 \mathrm{M}$ tris hcl, 0.01M EDTA Ph 7.5

10xLiAc 1M LiAc $\mathrm{Ph} 7.5$ adjusted with diluted acetic acid

7 Incubate at $30^{\circ} \mathrm{C}$ for $20 \mathrm{~min}$

8 Transfer cell $0.1 \mathrm{ml}$ to $1.5 \mathrm{ml}$ tube and centri at $14,000 \mathrm{rpm}$ for $30 \mathrm{~s}$ at RT. Decant the supernatant. These cells are ready for transformation.

Note Use Competent Cells within $1 \mathrm{~h}$ after preparing them

9 In $1.5 \mathrm{ml}$ tube combine the following

\begin{tabular}{|l|l|}
\hline Component & Volumn \\
\hline ds cDNA & $20 \mu \mathrm{l}$ \\
\hline AD:pGADT7-Rec $(0.5 \mu \mathrm{g} / \mu \mathrm{l})$ & $6 \mu \mathrm{l}=3 \mu \mathrm{g}$ \\
\hline BD:pGBKT7(with insert) & $5 \mu \mathrm{g}(<10 \mu \mathrm{l})$ \\
\hline $\begin{array}{l}\text { Herring Testes Carrier } \\
\text { DNA }\end{array}$ & $20 \mu \mathrm{l}$ \\
\cline { 1 - 1 } Boil $30 \mu \mathrm{l}$ in $100^{\circ} \mathrm{C} 20 \mathrm{~min}$ & \\
\hline
\end{tabular}

10 add $600 \mu l$ competent cell to DNA mix by Vertex gently

11 add freshly made $2.5 \mathrm{ml}$ PEG/LiAc Solution mix by Vertexing

PEG/LiAc solution(10ml): 8ml 50\%PEG, $1 \mathrm{ml}$ 10xTE, 1ml10xLiAc 1M.

12 incubate at $30^{\circ} \mathrm{C} 45 \mathrm{~min} \sim 1 \mathrm{~h}$, mix cells every $15 \mathrm{~min}$ 
13 add $80 \mu \mathrm{l}$ DMSO, mix incubate in $42^{\circ} \mathrm{C} 15 \mathrm{~min}$ then chill on ice for $5 \mathrm{~min}$

15 centri $700 \mathrm{~g}$ for $5 \mathrm{~min}$

16 Discard the supernatant and resuspend in $3 \mathrm{ml}$ of $0.9 \% \mathrm{NaCl}$

17 spray $200 \mu l$ to each selective plate(-Leu; -Trp; -Leu-Trp; -Leu-Trp-His) keep in $30^{\circ} \mathrm{C}$ $2 \mathrm{~d}$ 